STUDI E SAGGI

- 191 - 

Fortunato Tito Arecchi

\section{Cognizione e realtà}

FIRENZE UNIVERSITY PRESS

2018 
Cognizione e realtà / Fortunato Tito Arecchi. - Firenze : Firenze University Press, 2018.

(Studi e saggi ; 191)

http://digital.casalini.it/9788864537467

ISBN 978-88-6453-745-0 (print)

ISBN 978-88-6453-746-7 (online)

Progetto grafico di Alberto Pizarro Fernández, Pagina Maestra snc Immagine di copertina: $\odot$ Agsandrew $\mid$ Dreamstime.com

\section{Certificazione scientifica delle Opere}

Tutti i volumi pubblicati sono soggetti ad un processo di referaggio esterno di cui sono responsabili il Consiglio editoriale della FUP e i Consigli scientifici delle singole collane. Le opere pubblicate nel catalogo della FUP sono valutate e approvate dal Consiglio editoriale della casa editrice. Per una descrizione più analitica del processo di referaggio si rimanda ai documenti ufficiali pubblicati sul catalogo on-line della casa editrice (www.fupress.com).

\section{Consiglio editoriale Firenze University Press}

A. Dolfi (Presidente), M. Boddi, A. Bucelli, R. Casalbuoni, M. Garzaniti, M.C. Grisolia, P. Guarnieri, R. Lanfredini, A. Lenzi, P. Lo Nostro, G. Mari, A. Mariani, P.M. Mariano, S. Marinai, R. Minuti, P. Nanni, G. Nigro, A. Perulli, M.C. Torricelli.

La presente opera è rilasciata nei termini della licenza Creative Commons Attribution 4.0 International (CC BY 4.0: http://creativecommons.org/licenses/by/4.0/legalcode).

This book is printed on acid-free paper

Cc 2018 Firenze University Press Università degli Studi di Firenze Firenze University Press via Cittadella, 7, 50144 Firenze, Italy www.fupress.com 
PARTE A

COGNIZIONE E REALTÀ

1. Premessa 5

2. Percezione come inferenza di Bayes 7

3. Da percezione a giudizio: la coscienza $\quad 12$

4. Dal singolo colle di probabilità alla complessità semantica 13

5. Come nasce il linguaggio: Bayes inverso e salti non algoritmici 15

6. Il programma scientifico: probabilità promossa a certezza 21

7. Il ruolo dei processi linguistici nei programmi scientifici 26

8. Le due ermeneutiche: circolo e spira 28

9. Cognizione e Realtà: domande e risposte 32

PARTE B

LA FISICA $\quad 41$

1. Il programma della fisica 41

2. Complessità formale, algoritmica e semantica 44

3. Caos deterministico: esempi di applicazione a processi
percettivi e ad orologi biologici

4. Fisica e fisiologia $\quad 50$

5. Dinamica ed energia 52

6. Particelle e forze 59

7. Onde 61

8. Bilanci energetici: chimica e vita 64

9. I quanti 66

10. Spazio-tempo da Newton ad Einstein: le onde gravitazionali 73

$\begin{array}{ll}\text { 11. La fisica del disordine } & 76\end{array}$

12. Fisica dei processi percettivi e sincronizzazione di impulsi neuronali

PARTE C

$\begin{array}{ll}\text { DIZIONARIO RAGIONATO } & 91\end{array}$

$\begin{array}{lr}\text { NOTA BIBLIOGRAFICA } & 129\end{array}$

INDICE ANALITICO 133

Fortunato Tito Arecchi, Cognizione e realtà, ISBN 978-88-6453-745-0 (print) ISBN 978-88-6453-746-7 (online), 



\section{INTRODUZIONE}

Qualunque essere vivente si attiva a 'conoscere' il mondo, nel senso che reagisce agli stimoli che riceve in modo da ottimizzare la propria sopravvivenza. Chiameremo 'percezione' questo contatto con l'esterno che richiede una reazione.

Nella percezione, lo stimolo di ingresso viene confrontato con un archivio di possibili risposte e se ne sceglie la più opportuna, con una operazione che Thomas Bayes nel' 600 ha chiamato 'inferenza' e ha codificato in termini di probabilità. Questo archivio di possibili risposte è in parte eredità genetica, in parte appreso nella prima parte della vita, nelle varie esperienze.

Negli umani - però - c'è una cosa in più. Ci si domanda: come va il mondo senza di me? Chiamiamo 'realtà' questo comportamento, e 'giudizio' l'operazione che ci ha permesso di esplorare una realtà.

Nel giudizio, estendiamo il conoscere dallo stimolo percettivo all'operazione linguistica, confrontiamo cioè un brano di discorso con un brano precedente e ne estraiamo la migliore adeguazione, con una operazione che ho chiamato 'inferenza inversa di Bayes'.

L'adeguazione non è completata una volta per tutte - come sembrerebbe nella immagine a sinistra nella figura qui riportata (anticipo di quanto detto in dettaglio nella Fig. 29 della Parte A) - ma è migliorabile attraverso successive ripetizioni, come mostrato nella immagine a destra. Se poi l'oggetto del conoscere, cioè la realtà sotto osservazione, è un altro essere umano, allora le ripetute osservazioni non solo aumentano in me la sua conoscenza, ma stimolano l'altro a modificarsi di conseguenza: è nato così il dialogo fra due persone.
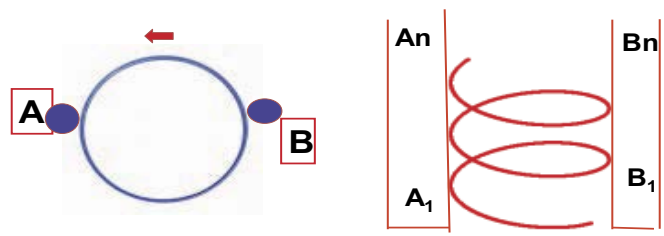

Confronto fra due modi di leggere il mondo, presentati in dettaglio nella figura 29 della Parte A e chiamati Ermeneutica ripetitiva ed Ermeneutica creativa. 
A questo punto, l'attenzione è rivolta non più al contatto con l'altro (la percezione) ma all'elaborazione linguistica, che porta al giudizio. Le operazioni del nostro cervello consistono in:

i) codifica di un elemento cognitivo (stimolo sensorio o classificazione già avvenuta) in sequenze ordinate di impulsi elettrici (spikes) dei neuroni cerebrali;

ii) confronto delle sequenze di spikes provenienti da regioni cerebrali diverse, cercando l'accordo (sincronizzazione dei treni di spikes) come adeguazione fra domanda e risposta.

Abbiamo introdotto dettagli che richiedono spiegazioni. I dettagli sui processi cognitivi, e in particolare linguistici, richiedono una conoscenza preliminare della fisica sottostante, in quanto si avvalgono degli strumenti elaborati dalla indagine fisica.

Nella Parte A del volume, in cui si sviluppa il tema suggerito dal titolo, la presentazione dei problemi e delle soluzioni è fatta appoggiandoci al ruolo euristico di immagini semplici; la sezione si conclude con alcune Domande e con le relative Risposte. Questa Parte, inoltre, è di per sé autosufficiente, ma fa uso di termini che richiedono una adeguata presentazione. Perciò al testo principale della Parte A seguono altre due Parti, che sono ausili pedagogici: - la Parte B è una presentazione dei concetti e delle elaborazioni della fisica, concetti ed elaborazioni che condizionano qualunque discorso scientifico;

- la Parte C è un conciso dizionario ragionato che introduce e chiarisce alcune nozioni tecniche utilizzate nella Parte A.

Chiude il libro una Nota bibliografica essenziale, che evita di proporre testi (articoli scientifici o libri) già citati nei testi suggeriti.

In conclusione, abbiamo sottolineato il ruolo unico della cognizione umana rispetto a quella degli altri viventi. Si pone una domanda cruciale: i progressi recenti nello sviluppo di macchine per AI (Intelligenza Artificiale) potranno un giorno portare a un sostituto cognitivo dell'uomo?

La risposta è no, per il seguente motivo: se l'azione linguistica consiste nella sincronizzazione ottimale dei codici neuronali di due brani di discorso messi a confronto, una macchina ottimizza la sincronizzazione quando le due sequenze sono uguali, pertanto arriva ad una ovvia identità; invece, negli umani, il codice del primo brano è sottoposto a stimoli emozionali, quindi la sincronizzazione è preceduta da una elaborazione del primo brano e pertanto essa rivela un significato nuovo!

Il desiderio di costruire un sapere che risponda ad un criterio di verità può spingere a diffidare dei riferimenti alle profondità invisibili della soggettività umana. Questo è quanto succede con il comportamentismo, che si propone di studiare soltanto il comportamento osservabile, vietandosi i resoconti introspettivi. La prassi dei calcolatori rende possibile concepi- 
re l'uomo come una macchina dotata di software. Il software è un'implementazione delle regole di ragionamento equivalenti ad operazioni esatte sui segni. L'impiego della metafora del pensiero come software è proprio del cognitivismo che si sostituisce al comportamentismo come orientamento dominante.

Abbiamo dunque un comportamentismo che vieta di fare riferimento al vissuto personale, e un cognitivismo che accetta di parlare dei mondi invisibili della soggettività, ma soltanto per coglierne i tratti di razionalità.

Ora, le strutture biologiche del cervello consistono di tre livelli: il livello primario che corrisponde alle risposte emotive grezze, il livello secondario composto dai meccanismi di memoria ed apprendimento, e il livello terziario in cui troviamo le complessità cognitive della riflessione.

Distinguiamo, annidati uno dentro l'altro, tre cervelli. Quelli più vicini alla colonna vertebrale (1-subcorticale; 2 -limbico) sono i più antichi, mentre quello più lontano (3-corteccia cerebrale o neo-corteccia) è il più recente. Vi sono ruoli diversi:

- processi primari: emozioni, sede subcorticale;

- processi secondari: apprendimento, sede limbico, influenza i pensieri;

- processi terziari: processi cognitivi, sede neocorteccia.

Per fissare le idee, possiamo esemplificare il livello primario con il terrore di fronte ad una tigre, il livello secondario con il ricordo dei segni del predatore, e il livello terziario con la discussione di un progetto per catturare la tigre. La localizzazione dei circuiti emotivi avviene inserendo degli elettrodi nel cervello per produrre una stimolazione elettrica in punti specifici.

Al riguardo, concludo questa nota introduttiva suggerendo un libro non riportato nella Nota bibliografica: A. Damasio, Emozione e coscienza (Adelphi, Milano 2000). Damasio dimostra come, al contrario di una tradizione culturale che ha sempre svalutato le emozioni perché perturberebbero la serenità della ragione, esse siano alla base del buon funzionamento della mente: se l'uomo perde la capacità emozionale non è in grado di essere ragionevole. Negando la concezione cartesiana del dualismo mente-corpo, egli mette in evidenza l'azione reciproca del corpo e del cervello. La ragione non potrebbe funzionare correttamente senza le emozioni.

Gli affetti non sono attitudini proposizionali derivate da valutazioni emotive. Difatti, il linguaggio non è il segno distintivo dell'affetto soprattutto perché non riesce a spiegare le esperienze primarie. Ad esempio, "'rosso" non ha alcun significato intrinseco, l'esperienza del rosso sì»e le parole non sarebbero mai in grado di spiegare l'esperienza del vedere il colore rosso a qualcuno che è cieco. Le parole sono solo etichette e non possono da sole spiegare l'esperienza percettiva primaria.

Il rapporto fra processi cognitivi e sfera delle emozioni costituisce uno dei capitoli più intricati della ricerca psicologica. Tale rapporto determina 
le attribuzioni di significato, ed è quello che ci fa 'umani' e vincenti rispetto all'intelligenza artificiale.

Ringrazio tutti coloro che hanno letto le versioni preliminari di questo testo ed hanno espresso dubbi o critiche contribuendo a migliorarne la presentazione. 
PARTE A

COGNIZIONE E REALTÀ

\section{Premessa}

Perché questo titolo? Perché Cognizione e non Conoscenza?

Conoscenza indica l'insieme delle cose conosciute, mentre Cognizione indica l'atto del conoscere cioè dell'attribuire un senso a stimoli sensoriali, determinando di conseguenza una reazione motoria; ed è questo che conto di esplorare in queste pagine.

Realtà (da res= cosa) denota situazioni indipendenti da noi, che hanno pertanto un'esistenza autonoma e non sono il risultato di nostri atti. Si discute se la cognizione catturi le realtà, o addirittura le costruisca.

Nel mito della caverna, Platone dice che ci accostiamo alle realtà come un prigioniero in una caverna costretto a vedere solo le ombre sulla parete di fondo. In effetti, le ombre a un certo istante danno un contributo parziale, e il confronto con le ombre viste a tempi successivi aumenta l'informazione su quanto accade.

Nella figura nell'Introduzione si sono messi a confronto i due modi di interpretare i processi cognitivi umani.

$A$ rappresenta il soggetto che conosce, $B$ l'oggetto del conoscere. A sinistra, $A$ si è fatta un'opinione precisa di $B$; ogni volta che torna a esplorare $B$, applica la stessa interpretazione: chiameremo ermeneutica a circolo questo intrecciarsi di $A$ e $B$, senza progressi. Invece, a destra, $A$ si forma una prima immagine di $B$ ( $A_{1}$ che legge $\left.B_{1}\right)$ ma ogni volta che $A$ ritorna a $B$, partendo da un punto di vista più evoluto, ad esempio $A_{2}$, scopre ulteriori connotazioni per cui $B_{2}$ è più ricco di informazione rispetto a $B_{1}$. Chiameremo questo arricchimento graduale ermeneutica a spira.

Se poi $B$ è un altro essere umano dotato a sua volta di capacità linguistiche, allora le successive connotazioni di $B$ non sono solo dovute alla cresciuta comprensione da parte di $A$, ma anche al fatto che il dialogo fra $A$ e $B$ fa crescere non solo $A$ ma anche $B$, che risponde alle domande di $A$ e si modifica di conseguenza: è quello che chiamiamo dialogo inter-personale.

Il confronto fra le due ermeneutiche presuppone l'abilità linguistica, cioè la capacità di codificare le percezioni in un linguaggio e mettere a confronto brani successivi di un testo (come quando ascoltiamo una poesia o un testo musicale). Dunque nella figura si mettono a confronto due diverse procedure linguistiche. 
Ma allora, conoscere implica un linguaggio? E tutti gli altri animali con cervello, ma che non hanno elaborato un linguaggio?

A questo punto occorre aver presente una distinzione cruciale. Le operazioni cognitive sono di due tipi.

1. Le percezioni, in base alle quali stimoli sensoriali attivano reazioni motorie; le percezioni sono comuni a tutti gli animali con cervello. Lo stimolo (che chiamiamo $d=$ datum) suscita un ventaglio di possibili ipotesi interpretative (che chiameremo $h$, da hypothesis). E qui nasce il meccanismo di scelta: ogni $h$ evoca un possibile $d$ che l'ha generata, attraverso l'aspettativa probabilistica che $d$ sia conseguenza di un $h$. D'ora in poi, denotiamo il meccanismo di scelta con $P(d \mid h)$ (che chiamiamo probabilità condizionata che $d$ sia conseguenza di $h$ ). In corrispondenza al massimo di $P(d \mid h)$, la $h$ che lo ha generato (e che denoteremo con $h^{*}$ ) rappresenta l'ipotesi più plausibile, in base a cui il cervello elabora una reazione appropriata (fuga da pericolo, cattura di cibo).

Il $P(d \mid h)$ è lo strumento interpretativo di cui è dotato ogni cervello. Esso è un'eredità genetica o il risultato di una fase di apprendimento infantile ed è conservato nella memoria a lungo termine. Lo chiameremo algoritmo.

2. Invece, un linguaggio (letterario, musicale, figurativo) codifica un evento cognitivo in un testo, che acquista significato dal confronto di un brano con brani precedenti per estrarne il senso più soddisfacente; questo confronto non implica un algoritmo $P(d \mid h)$ perché non implica ventagli di aspettative, in quanto sia il brano che precede sia quello che segue sono già strutturati; piuttosto il confronto genera la migliore interpretazione del secondo brano in virtù del primo: dunque, invece di invocare un $P(d \mid h)$ pre-esistente, qui emerge un

\section{$P\left(2^{\circ} \mid 1^{\circ}\right)$ che rappresenta un giudizio.}

La singola osservazione rappresenta una percezione o apprensione; essa non implica ancora un problema di realtà se non inserita in un testo $\mathrm{e}$ confrontata con osservazioni precedenti.

Invece, l'elaborazione linguistica cattura aspetti sempre più dettagliati della realtà; non si conosce la realtà una volta per tutte, ma si instaura un dialogo senza fine, acquisendo ogni volta ulteriori aspetti.

Si corregge in tal modo il limite del prigioniero di Platone, in quanto abbiamo la possibilità di variare il punto di vista, catturando punti di vista sfuggiti alla singola apprensione; tale è anche il rapporto fra persone, che un'ermeneutica ripetitiva credeva di congelare in una singola osservazione.

La differenza cruciale fra percezione e giudizio è stata elaborata da Bernard Lonergan in Insight (1957) come risposta a filosofie della conoscenza che non chiarivano il problema, ma lo complicavano con soluzioni dubbie. La percezione non implica il linguaggio, ma consiste nell'interpretare uno stimolo sensoriale in vista di una reazione vitale; si tratta di un processo comune a qualunque animale con cervello e si può descrivere come una 
inferenza di Bayes in cui l'algoritmo interpretativo è immagazzinato nella memoria a lungo termine (quella che dura tutta la vita). Il giudizio consiste invece nel confronto fra due percezioni codificate in un linguaggio (letterario, musicale, figurativo); la precedente viene ripresentata dalla memoria a breve termine (che opera sui 3 secondi) e confrontata con la successiva. L'operazione - chiamata Bayes inverso - non presuppone un algoritmo, ma ne costruisce uno nuovo attraverso il confronto.

Vedremo come i salti di algoritmo legati a operazioni linguistiche catturino aspetti della realtà non raggiungibili attraverso catene di inferenze di Bayes ricorsive (cioè ciascuna che parte dalla precedente) guidate dallo stesso algoritmo.

Dunque, mentre nella percezione si elabora il dato esterno con un algoritmo, nella procedura linguistica si confrontano due brani di un testo, acquisiti a tempi diversi. In questo secondo caso, nel fare il confronto, è cruciale che l'attore linguistico che richiama il brano precedente sappia di essere lo stesso che presenta il brano seguente, sì che dal confronto emerga il Bayes inverso. Dunque, la coscienza di se stessi, base delle decisioni etiche, è legata alla elaborazione linguistica ed è solo umana. Invece la consapevolezza percettiva (è accaduto qualcosa a cui devo reagire) è comune a tutti gli animali con cervello.

La scienza moderna nata dalla rivoluzione galileiana («non tentare le essenze, ma contentarsi delle affezioni quantitative») viene vista come una procedura linguistica che elimina quelle ambiguità che richiedono visitazioni ripetute dell'oggetto del conoscere, come avviene nei giudizi etici.

Oggi sperimentiamo i successi dell'AI (Intelligenza Artificiale) che opera con algoritmi potenti; ma l'AI opera per Bayes diretto e non fa i salti algoritmici peculiari dei linguaggi umani; essa velocizza le catene ricorsive ma non ricorre a salti algoritmici. Dunque, l'AI e i robot che ne conseguono, velocizzala consapevolezza, ma non abilita alla coscienza di sé. Non dobbiamo pertanto temere che l'AI sostituisca il linguaggio umano.

\section{Percezione come inferenza di Bayes}

Qualunque animale con cervello coordina i diversi stimoli sensoriali attraverso strumenti interpretativi (d'ora in poi chiamati algoritmi) in base ai quali trasmette agli organi motori una adeguata reazione. Facciamo un esempio: un coniglio sente un fruscio dietro una siepe e scappa: lo chiamiamo 'istinto di sopravvivenza'; in effetti, un algoritmo ha classificato le sensazioni indotte dal fruscio come presenza di un pericolo e ha suggerito la fuga. Lalgoritmo è stato ereditato geneticamente o costruito nella fase infantile di apprendimento; esso dura tutta la vita (memoria a lungo termine).

Invece, un umano dotato di linguaggio immagazzina la prima percezione, la classifica in un adeguato linguaggio e la confronta con una ulte- 
riore percezione classificata nello stesso linguaggio; il confronto implica una ripresentazione della prima percezione e richiede - come vedremo un tempo di circa 3 secondi corrispondente a una memoria a breve termine. In tal modo, il confronto fra due percezioni corrispondenti a punti di vista diversi può stabilire se il fruscio era dovuto ad una volpe in agguato o semplicemente a un colpo di vento.

Dunque, sembra che una singola percezione non sia sufficiente a catturare la realtà (cioè, lo stato delle cose indipendente dal nostro percepire).

Fermandosi a questo livello e in mancanza di una adeguata analisi dei processi linguistici, Platone formulò il mito del prigioniero legato all'imbocco di una caverna e costretto a vedere solo le ombre proiettate dal sole sul fondo e ad agire di conseguenza (Fig. 1).

Il futuro calcolato dipende criticamente dall'algoritmo, ma non è detto rappresenti la realtà.

\section{Mito platonico della caverna (singola visione)}

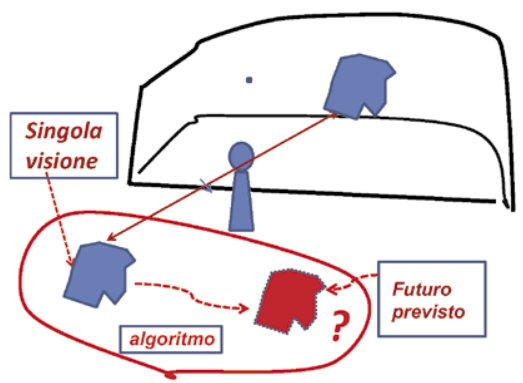

Figura 1 - Il mito platonico della caverna: il prigioniero legato può solo vedere il fondo della caverna e le ombre degli eventi esterni proiettate sul fondo della caverna; mediante algoritmi immagazzinati nella memoria a lungo termine interpreta queste ombre e ne estrae una adeguata reazione motoria (corrispondente a un calcolo sul futuro).

In figura 2, nei primi stadi corticali le cellule sono specializzate per catturare un aspetto particolare; per esempio, alcuni neuroni si attivano (cioè scaricano impulsi elettrici) solo se illuminati da barre verticali, altri sentono barre orizzontali, altri barre con una certa inclinazione; l'oggetto visivo, frantumato nei suoi aspetti salienti, va ricostituito dal confronto fra i vari gruppi.

Abbiamo chiamato 'stadi corticali alti' la zona della corteccia cerebrale dove confluiscono i segnali provenienti da diversi sensi (vista, udito, olfatto, tatto); questa zona corrisponde alla PFC (pre-frontal cortex $=$ corteccia pre-frontale); genericamente l'area in cui si unificano le sensazioni e ha luogo il confronto con le memorie a lungo termine per stabilire l'opportuna risposta è stata chiamata GWS (global work-space). Uno schema preliminare di Percezione è presentato in figura 3. 


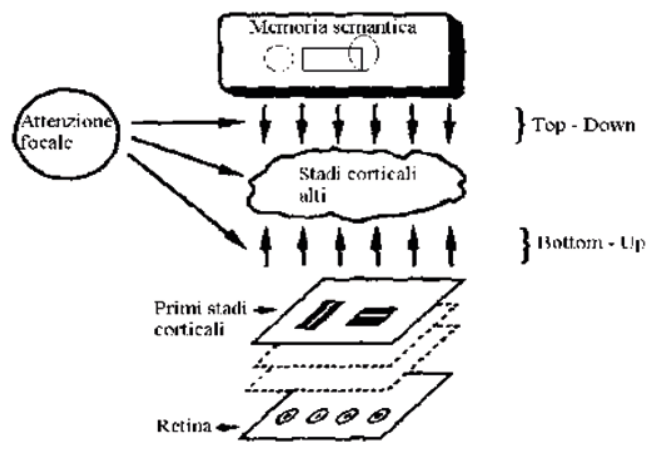

Figura 2 - Cooperazione fra stimoli e categorie memorizzate per formare una percezione: gli organi sensori periferici (retina nel caso visivo) ricevono stimoli dall'esterno; gli elementi salienti vengono rivelati da cellule specializzate e convogliati agli stadi corticali alti (bottom- $u p=$ percorso dal basso verso l'alto); qui vengono 'interpretati' da algoritmi conservati nella memoria semantica e che intervengono top-down (dall'alto verso il basso). Un meccanismo di 'attenzione focale' assicura questa interpretazione; il risultato è una reazione motoria allo stimolo.

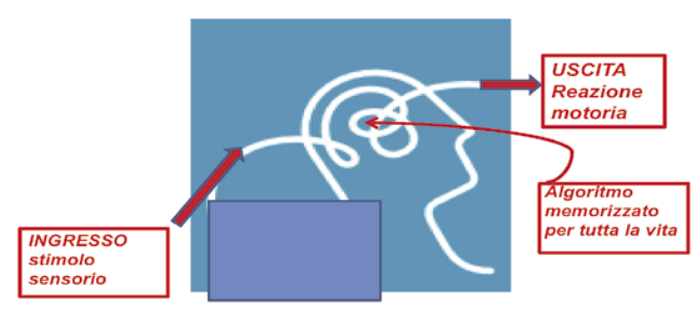

Figura 3 - La figura riassume quanto illustrato nello schema di figura 2; il tempo richiesto dal processo è una frazione di secondo; la percezione riguarda qualunque animale con cervello, dal verme $C$. aelegans, che ha 300 neuroni, all'uomo, il cui cervello ha 1012 (mille miliardi) di neuroni. 


\section{Apprensione (inferenza di Bayes)}

( $h$ = hypothesis; $d=$ data)

Selezione di $h^{\star}$ partendo da un pacchetto di $h$, per azione congiunta di uno stimolo sensorio (bottom-up) e di un modello interpretativo (top-down)

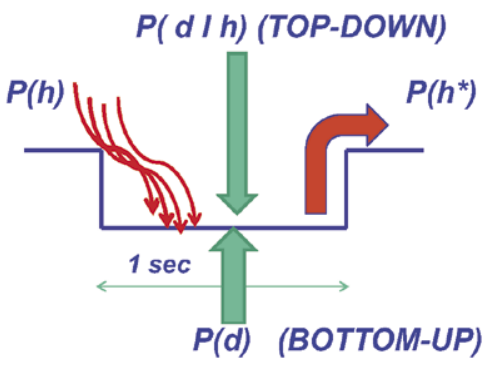

Figura 4 - Chiamiamo Apprensione la percezione che ha unificato i differenti canali sensoriali in un dato $d$ che arriva con probabilità $P(d)$ bottom-up (dal basso verso l'alto). Il dato suscita aspettative $h$ (da hypothesis = ipotesi) diverse, ciascuna con probabilità $P(h)$. Lalgoritmo interpretativo (ereditato geneticamente o dovuto ad apprendimenti dell'età infantile; dura tutta la vita; viene richiamato dalla cosiddetta memoria a lungo termine) consiste nella probabilità condizionata $P(d \mid h)$ che ad una ipotesi $h$ consegua il dato $d$. Il confronto con il dato effettivo seleziona la $h^{*}$ vincente con probabilità $P\left(h^{\star}\right)$. Basandosi su $P\left(h^{*}\right)$, il cervello invia segnali ai canali motori, suscitando una adeguata reazione (la fuga nel caso del coniglio).

Come illustrato in figura 4, il risultato $P\left(h^{*}\right)$ della percezione è la probabilità condizionata $P(h \mid d)$ di una ipotesi in presenza di un dato, cioè:

$$
P(h \mid d)=\frac{P(h) P(d \mid h)}{P(d)}
$$

Lo strumento interpretativo $P(d \mid h)$ è anch'esso una probabilità; per contro, nella fisica di Galileo e Newton, il dato che emerge è certo, cioè:

$$
P(d \mid h)=1
$$

come spiegato nella Parte C.

Per capire come funziona Bayes, in figura 5 presentiamo uno spazio a 3 dimensioni: ogni punto del piano orizzontale rappresenta la misura di un evento; l'asse verticale rappresenta la probabilità associata ad ogni punto del piano; la linea rossa rappresenta l'algoritmo $P(d \mid h)$. La figura mostra una sequenza di applicazioni successive, detta ricorsiva, perché ogni applicazione parte dalla precedente. 
Successive applicazioni (ricorsive) di Bayes = scalata con le probabilità [es. Darwin, Sherlock Holmes, 'sistemi esperti']

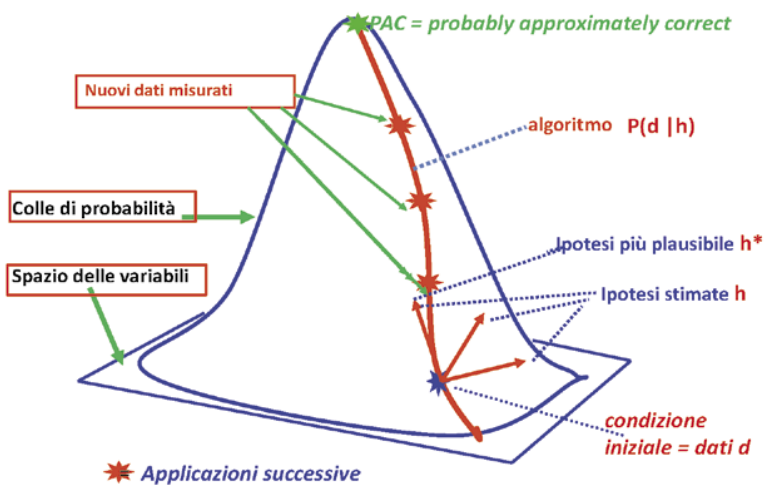

Figura 5 - Partendo da una condizione iniziale con bassa probabilità ( ${ }^{*}$ in basso), si formulano diverse ipotesi( le frecce); di queste, una $\left(h^{\star}\right)$ si adegua meglio all'algoritmo e ci porta a una probabilità più alta (seconda ${ }^{\star}$ dal basso). Da qui, si effettuano nuove misure e si ripete loperazione bayesiana (parleremo di applicazione ricorsiva), finché raggiungiamo il massimo di probabilità. Possiamo parlare di certezza, di aver catturato una realtà? No! Dato il carattere soggettivo della procedura bayesiana, parleremo di conoscenza più probabile (PACknowledge = conoscenza "probably approximately correct»).

La procedura bayesiana, introdotta a fine Settecento, ha influenzato tutta la scienza dell'Ottocento.

L'evoluzione di Darwin, come coppia di mutazione e selezione, è bayesiana: l'algoritmo rappresenta l'adattamento di una specie alla biosfera; la pluralità di ipotesi ad ogni passo (indicato nella figura con ${ }^{*}$ ) rappresenta le mutazioni che intervengono nei genotipi (ad esempio, per bombardamento da raggi cosmici), la selezione di $h^{\star}$ equivale al successo di un particolare genotipo, il cui fenotipo arriva all'età adulta e genera prole.

Anche l'indagine di Sherlock Holmes è bayesiana: l'algoritmo è il modello del crimine formulato dall'investigatore; i dati sono gli indizi raccolti durante l'indagine. Ad ogni scalino in alto aumenta la probabilità e il punto di arrivo $\left({ }^{*}\right)$ diventa la partenza per una ulteriore sessione con ulteriori dati raccolti.

La scienza 'normale' (uso la distinzione di Kuhn fra scienza normale e salto di paradigma, quest'ultimo corrispondente - come vedremo - all'introduzione di un nuovo algoritmo) è bayesiana. Riassumiamo la percezione come inferenza di Bayes nella quadrupla:

$$
\text { stimolo }=d ; \text { congettura }=h ; \text { algoritmo }=P(d \mid h) ; \text { risposta }=h^{*}
$$


Dunque, nella scienza normale avremo:

stimolo $=$ misura $;$ algoritmo $=$ teoria $($ ad es. Newton $) ;$ risposta $=$ risultato

\section{Da percezione a giudizio: la coscienza}

La percezione è la base della nostra vita di relazione. Essa è il primo dei due processi cognitivi esplorati da Bernard Lonergan in Insight.

I) Percezione = reazione sul mondo di un animale con cervello: gli stimoli sensoriali sono interpretati mediante algoritmi immagazzinati nella memoria a lungo termine determinando una reazione motoria (richiede circa 1sec).

Il secondo processo è il seguente.

II) Giudizio= lettura del mondo (solo umana): in una impresa linguisti$\mathrm{ca}$, un brano di un testo richiamato dalla memoria a breve termine (circa 3 sec) viene confrontato con il successivo; il confronto estrae il significato degli elementi linguistici (parole, note musicali, chiazze di colore) più appropriato per connettere brani successivi del discorso. In un animale o in un robot (o qualunque dispositivo di $\mathrm{AI}=$ intelligenza artificiale) la conoscenza consiste nel riconoscimento da un insieme finito di stadi o istruzioni precedenti.

Nell'uomo, il linguaggio «fa un uso infinito di un insieme finito di risorse» (W. von Humboldt); vedremo che ciò implica un salto di algoritmo. Il confronto fra i vari possibili significati di un testo richiede la consapevolezza che le varie presentazioni sono giudicate dallo stesso giudice (Fig. 6).

\footnotetext{
Procedura linguistica (solo in umani) $=$ testo codificato viene confrontato con testo precedente, richiamato da memoria a breve termine (fra 1 e $3 \mathrm{sec}$ ), dopo essere stato interpretato da 'emozioni'
}

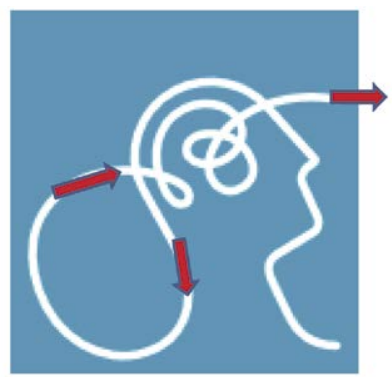

Figura 6 - Il confronto con figura 3 visualizza la differenza fra percezione e procedura linguistica.

Chiamiamo coscienza questa consapevolezza; essa è distinta dalla consapevolezza percettiva (il realizzare che vediamo questo e non quest'altro) che 
è stata esplorata con indicatori convenienti che hanno introdotto il concetto di NCC (neural correlates of consciousness = correlati neuronali della consapevolezza percettiva, impropriamente promossa a coscienza) (Francis Crick e Christophe Koch). Come vedremo, il giudizio ha luogo per 'Bayes inverso'.

Riassumiamo le due scale temporali dei processi cognitivi. Dettagli più accurati sono forniti nelle voci nel glossario della Parte C.

a) Apprensione (durata circa 1sec): è una percezione coerente in grado di indurre reazione motoria(procedura a repertorio finito, comune agli animali).

b) Giudizio (tempo caratteristico attorno ai $3 \mathrm{sec}$ ): è il confronto linguistico fra il brano presente e la memoria del brano precedente (procedura libera, creativa, solo umana).

In b) i due eventi sono codificati nello stesso linguaggio e sottoposti allo stesso giudice (coscienza di sé). L’operazione implica Bayes inverso.

Se sottoponiamo un soggetto all'ascolto di un testo poetico o musicale e gli chiediamo di classificare i tempi che intercorrono fra due pause, vediamo che la durata più frequente è attorno ai $3 \mathrm{sec}$ (Fig. 7). Lo stesso accade per 'pause' oculari (cioè durata della fissazione di un dettaglio) di fronte a un'opera figurativa (vedi Parte C).
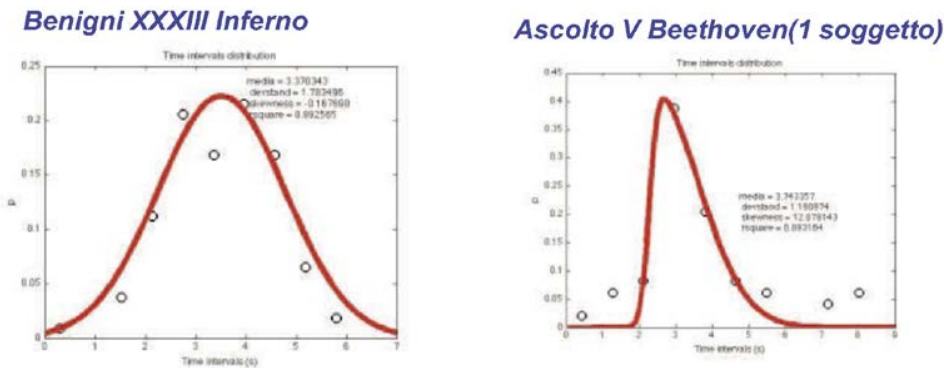

Figura 7 - Tempi di pausa mediati su molti soggetti (brani poetici o brani musicali). Facendo ascoltare un Canto di Dante o il primo movimento della V Sinfonia di Beethoven, si richiede al soggetto di classificare i tempi che intercorrono fra le pause; in entrambi i casi si hanno distribuzioni statistiche con massimo attorno ai 3 secondi.

\section{Dal singolo colle di probabilità alla complessità semantica}

Dato un algoritmo, chiameremo complessità algoritmica la pesantezza del calcolo da effettuare per applicare l'algoritmo; cioè misuriamo la lunghezza del programma di calcolo che rappresenta l'algoritmo in cifre binarie (i cosiddetti bit). Essa si riferisce a un singolo algoritmo (o a varianti di esso, tipo 'algoritmi genetici' di Holland; vedi Parte C). Per contro, diremo complessità semantica l'evidenza di molti colli di probabilità corrispondenti a diversi algoritmi. 
Nelle figure che seguono rappresentiamo rispettivamente:

- il paesaggio di probabilità con colli multipli (Fig. 8);

- la visualizzazione del teorema di Goedel (Fig. 9);

- le procedure che Kuhn ha chiamato scienza normale e salto di paradigma (Fig. 10).

Il salto da un colle all'altro in figura 8 (linee tratteggiate orizzontali) è non algoritmico. Esso corrisponde a una creatività propria del linguaggio, quando si fuoriesce dal sentiero della singola percezione: il matematico che scopre 'verità' non deducibili dagli assiomi (Fig. 9) sta operando con salti non algoritmici.

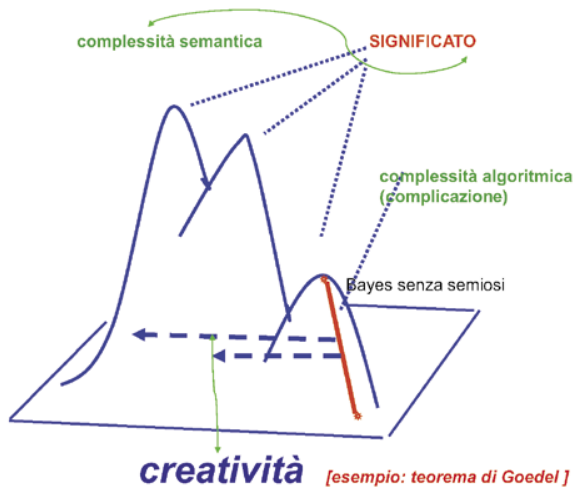

Figura 8 - Confronto fra complessità algoritmica (costo della scalata bayesiana di un singolo colle come in figura 5) e complessità semantica (esistenza di altri colli, con differenti algoritmi).

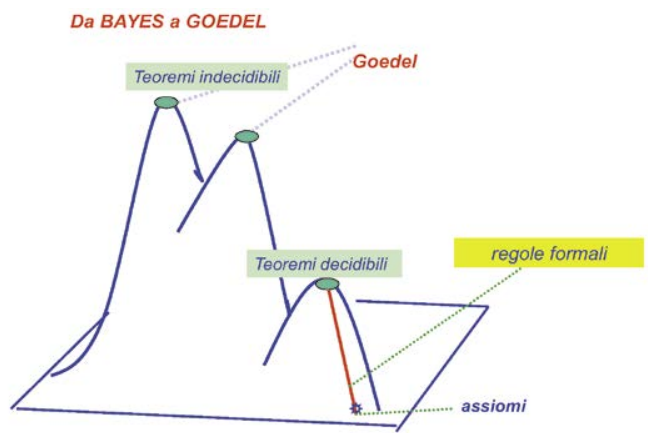

Figura 9 - Un teorema di Goedel del 1931 pone fine al sogno di Hilbert di costruire tutti i teoremi di una teoria matematica partendo dagli assiomi e applicando uno specifico algoritmo (cioè, le regole della logica formale). Invece Goedel ha mostrato che esistono 'verità, cioè teoremi, compatibili con gli assiomi, ma non 'decidibili' usando il primo algoritmo. La matematica è dunque affetta da complessità semantica. 


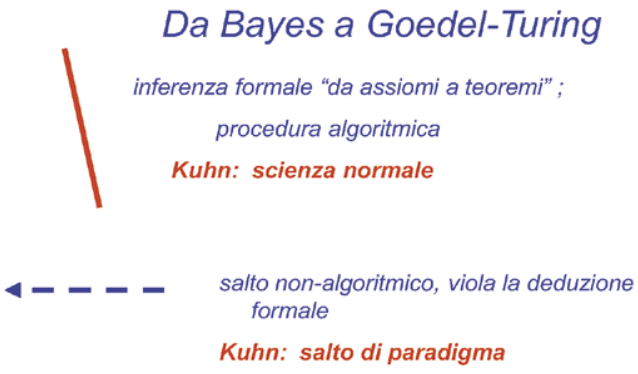

Figura 10 - Usando la terminologia introdotta da Kuhn, chiameremo 'scienza normale' quella che procede in modo bayesiano entro uno specificato algoritmo; mentre chiameremo 'salto di paradigma' il passaggio a un altro colle di probabilità con variazione di algoritmo. Un esempio: dopo le equazioni di Maxwell dell'elettromagnetismo (1863), radio e televisione sono applicazioni di scienza normale; invece il laser implica un salto di paradigma (il principio quantistico di emissione stimolata di Einstein, 1915).

Abbiamo riassunto in figura 10 la differenza fra procedura algoritmica e salto non algoritmico. Nella Struttura delle rivoluzioni scientifiche, Thomas Kuhn ha identificato le due procedure rispettivamente con la normale catena deduttiva all'interno di una scienza (scienza normale) e con l'introduzione di nuove leggi (salto di paradigma).

\section{Come nasce il linguaggio: Bayes inverso e salti non algoritmici}

Come abbiamo accennato, l'introduzione di codici linguistici (narrazione verbale, musica, pittura) consiste in questo: due brani successivi sono codificati nello stesso linguaggio e sottoposti allo stesso giudice (coscien$z a$ di sé) che esprime un parere di congruenza. Si tratta di una procedura libera, creativa; noi non attribuiamo un senso alla singola parola, ma al contesto, cioè al confronto fra una parola e le vicine.

Ciascuno può fare la verifica su se stesso, leggendo il testo di figura 11, in cui la singola parola sembra priva di senso in quanto le lettere che la compongono sono state rimescolate. Connettendo i brani del testo, si finisce con l'estrarne il senso, malgrado la distorsione delle singole parole.

La figura 12 corregge il pessimismo del mito platonico: il rivisitare un quid - che nella singola percezione non potevamo ancora chiamare 'realtà' - permette di estrarne molteplici punti di vista, eliminando le incertezze soggettive della singola percezione e dandoci assicurazione sulla sua 'realtà', cioè sulla sua consistenza non dipendente dal versante da cui era avvenuta la singola percezione.

Il confronto linguistico fra due elementi $d 2$ e $d_{1}$ di un testo, confrontati a distanza di circa $3 \mathrm{sec}$, fa emergere la probabilità condizionata $P\left(d_{2} \mid d_{1}\right)$, 
mediante una semplice operazione che consiste nello scambiare di posizione gli elementi delle identità di Bayes e che chiameremo Bayes inverso.

Il ruolo dei $3 \mathrm{sec}$ nei processi linguistici, come intervallo temporale diverso da quello percettivo (circa $1 \mathrm{sec}$ ) è stato esplorato dallo psicologo Ernst Pöppel a partire dal 1997.

Il Bayes inverso è stato da me introdotto nel 2010 in Dynamics of consciousness: complexity and creativity.

\section{Metodi di osservazione}

- Sneocdo uno sdtiuo dlel'Untisverià di Cadmbrige, non irmptoa cmoe snoo sctrite le plaroe, tutte le letetre posnsoo esesre al pstoo sbgalaito, è ipmtortane sloo che la prmia e l'umltia letrtea saino al ptoso gtsiuo, il rteso non ctona. II cerlvelo è comquune semrpe in gdrao di decraifre ttuo qtueso coas, pcheré non Igege ongi silngoa Itetrea, ma lgege la palroa nel suo insmiee.

Figura 11 - Testo in cui la singola parola appare non familiare, priva di senso, ma in cui il confronto con le altre parole permette correzioni che finiscono con il darle un senso.

\section{Mito della caverna (visioni multiple) ed elaborazione linguistica}

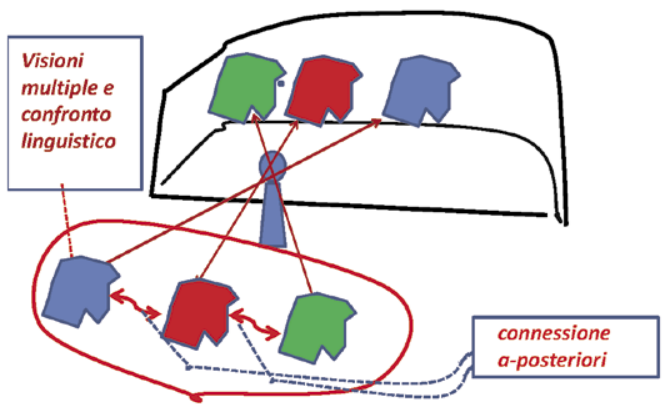

Figura 12 - Il mito platonico di figura 1 corretto dal programma linguistico. Visioni multiple, raccolte a tempi diversi, codificate in un linguaggio, vengono confrontate; dal confronto emerge un nuovo algoritmo, non pre-esistente nella memoria a lungo termine, e che giustifica la congruenza fra le visioni successive, che perciò danno assicurazione di indicare aspetti diversi della stessa realtà. 
Vediamo come opera in un testo poetico che consiste di una sequenza di versi: [...v5-v6-v7...].

Dal confronto fra v5 e v6 scaturisce la probabilità di v6 condizionata da v5, cioè:

$$
P(v 6 \mid v 5)=P(v 5, v 6) / P(v 5)
$$

L'operazione è visualizzata nelle figure 13 e 14 .

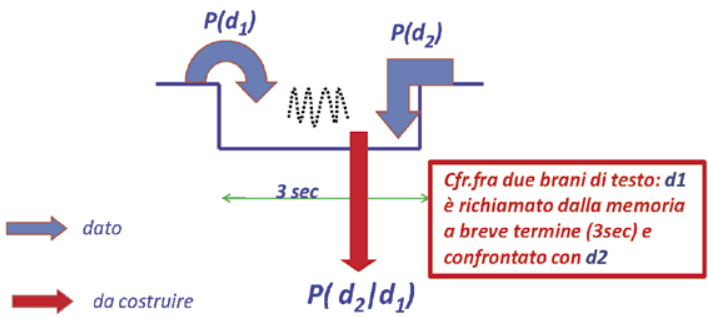

Figura 13 - Il giudizio come Bayes inverso. Confronto di $d 2$ e $d 1$ da cui emerge il legame più adeguato (decisione) (Arecchi 2010).

Bayes diretto
L'algoritmo deve essere noto
$P\left(h^{*}\right)=P(h)[P(d \mid h) / P(d)]$
scienza = inferenza di Bayes; algoritmo = teoria (Newton Einstein)
***
Bayes inverso
$P(d \mid h)=P(d) \times P\left(h^{*}\right) / P(h)$

Figura 14 - Confronto fra Bayes diretto (presuppone un algoritmo) e Bayes inverso (crea un algoritmo nuovo).

Se $d$ e $h^{*}$ non sono singoli termini lessicali, ma brani strutturati in un testo (letterario, musicale...) allora il $P(d \mid h)$ emergente rappresenta la migliore interpretazione. Il Bayes inverso riguarda non solo il confronto fra due brani successivi di un discorso, ma prima ancora la formulazione dei 
concetti. In tal caso, si ha un discorso interno in cui la definizione nominale $h^{*}$ viene applicata a esperienze successive $d$, 'nominate', cioè espresse con parole.

Il $P(d \mid h)$ risultante rappresenta la più adeguata lettura di $d$ in termini di $h^{*}$, cioè la scoperta di un concetto; per dirla con Tommaso d'Aquino, stiamo arrivando ad una verità come «adaequatio intellectus $\left(h^{*}\right)$ et rei $(d)$ ».

Per esseri umani, c'è una novità legata al linguaggio, per cui la coppia

\section{stimolo-memoria}

della percezione diventa la coppia

\section{$1^{\circ}$ brano- $2^{\circ}$ brano}

di un testo linguistico.

Invece di risposta motoria, abbiamo ora un:

'giudizio' = decisione sui significati del $2^{\circ}$ brano in base ai significati del $1^{\circ}$.

Questa sembra essere la novità che caratterizza il comportamento umano rispetto a quello di un animale privo di capacità linguistiche.

Dal punto di vista delle dinamiche neuronali (vedi Parti B e C) l'esplorazione dei significati del $1^{\circ}$ brano, per scegliere quelli che più si conformino al $2^{\circ}$, corrisponde a prospettarsi diverse sequenze di impulsi neuronali fino a scegliere la sequenza che assicura la migliore sincronizzazione.

Qui interviene un elemento nuovo, cioè la coscienza di se stessi: colui che sceglie il significato del $1^{\circ}$ brano deve essere consapevole di essere lo stesso cui si presenta il $2^{\circ}$.

In base a ciò si parla di Rivoluzione umana: la ricerca dei significati va oltre la lotta per la vita (base della percezione in animali con cervello).

Nell'introdurre il Bayes inverso, abbiamo invocato la coscienza di sé come condizione necessaria per confrontare i due brani testuali successivi. Sull'intervallo temporale della transazione linguistica non esistono misure di laboratorio che connettano i $3 \mathrm{sec}$. È perciò che consideriamo la coscienza di sé come distinta da NCC.

È stato dibattuto il ruolo ai fini della coscienza dei neuroni specchio scoperti da Giacomo Rizzolatti. In effetti, i neuroni specchio non sono esclusivi degli umani, ma sono presenti nei primati e sembrano rappresentare lo strumento di un legame sociale. In ogni caso, rientrano entro NCC e il loro ruolo riguarda l'ambito temporale dei processi percettivi (attorno a $1 \mathrm{sec}$ ).

In figura 15 distinguiamo fra:

- operazioni mentali (apprensioni) viste come Bayes diretto e misurabili con NCC, e

- operazioni composte dal confronto di un'apprensione (un verso di una poesia) con la precedente richiamata dalla memoria; in queste opera- 
zioni, che richiedono il linguaggio, si estrae il confronto per Bayes inverso. A tali operazioni composte non è associabile un NCC.

a)-APPRENSIONE ( Bayes diretto)

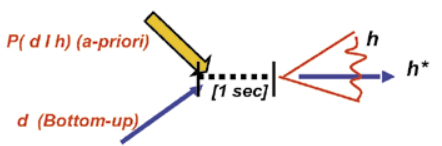

(NCC!)

b) GIUDIZIO (Bayes inverso)

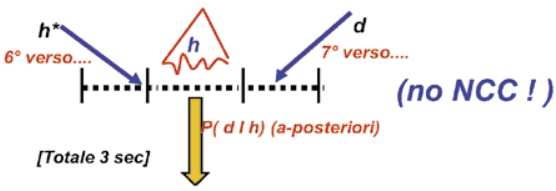

Figura 15 - a) Apprensione $=$ percezione con reazione motoria; dura circa 1sec; NCC $=$ neural correlates of consciousness, esplorati da Crick e Koch. Lapprensione corrisponde a selezionare $h^{\star}$ da un ventaglio di ipotesi $h$. Lo NCC si estrae da misure di FMRi (imaging [visualizzazione] mediante Risonanza Magnetica Funzionale) su soggetti durante la fase di Apprensione. b) Giudizio = attribuzione di un legame $P(d \mid h)$ al confronto fra due brani $d$ e $h^{\star}$ di un testo linguistico; il processo richiede 3 sec e su questa scala non esistono misure di NCC.

In figura 16 si elencano i cosiddetti neuro-derivati (neuro-etica, neuro-estetica, neuro-economia ecc.) consistenti nella presunzione di dedurre le decisioni in quel campo specifico da processi neuronali misurabili e traducibili in NCC. Si sta erroneamente attribuendo all'attività neuronale misurata nelle percezioni un ruolo decisionale che invece riguarda gli aspetti linguistici e che pertanto richiede il Bayes inverso.

Contro il punto di vista riduzionista (F. Crick, C. Koch) legato ai NCC (Neural Correlates of Consciousness); equivalente a 'c'è una regione del cervello per ...' = localizzazione per risonanza magnetica (fMRI), per cui:

$$
\text { 'neuro'\{ }\left\{\begin{array}{c}
\text { etica } \\
\text { estetica } \\
\text { economia } \\
\text { teologia } \\
\text { matematica } \\
\text { ecc. }
\end{array}\right.
$$

Invece per la 'fenomenologia' (da Husserl a Varela) non ha senso studiare il cervello isolato, ma tutto l'essere cognitivo in dialogo con il mondo.

Il Bayes inverso risulta da un confronto di significati, cioè da un dialogo e non da una singola emozione (quale evidenziata da fMRI)

Figura 16 - Oggi si parla di neuro-etica, neuro-estetica, neuro-economia ecc., attribuendo agli atti linguistici su cui si costruisce una decisione, base di ciascuna delle discipline elencate, una radice percettiva e ignorando il ruolo essenziale del Bayes inverso. Pertanto le neuro-xxxxx vanno considerate come errori scientifici. 
Abbiamo visto la peculiarità del linguaggio umano rispetto alle limitate percezioni degli altri animali con cervello. Ci domandiamo: da quanto tempo si è sviluppato questo potere?

Se esploriamo il passato preistorico, non abbiamo evidenze di testi letterari o musicali, ma i dipinti rupestri (Figg. 17 e 18) ci portano a contatto con nostri simili che 40.000 anni orsono leggevano il mondo come noi, con un linguaggio che è attuale anche oggi.

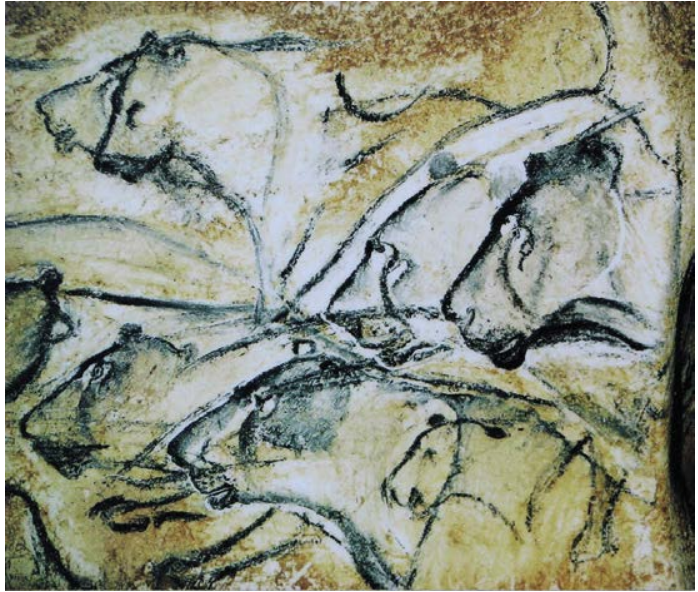

Figura 17 - Dipinto rupestre di 40.000 anni di un corteo di leoni nella Caverna Chauvet.

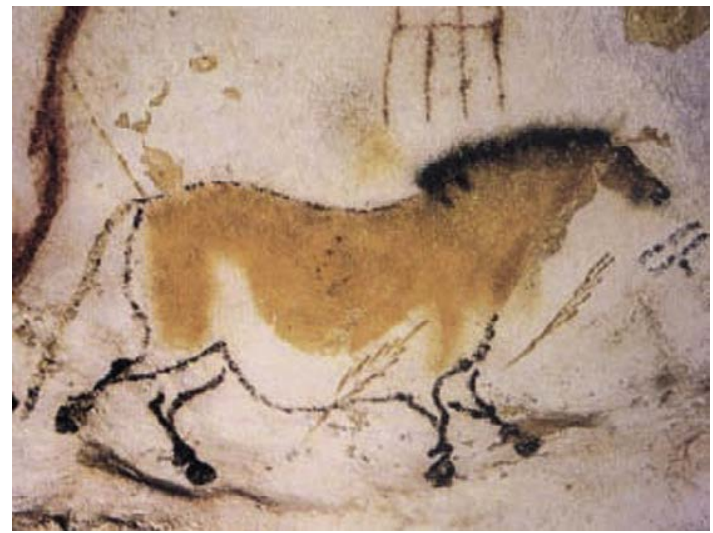

Figura 18 - Dipinto rupestre di un cavallo nella Caverna di Lascaux: come la mente umana ha astratto i significati. 
Nell'ambito della procedura linguistica, il punto di vista introdotto da Galileo (Fig. 19) rappresenta una rivoluzione linguistica che segna la nascita della scienza moderna. Si opera sempre per Bayes inverso, ma la prima configurazione viene confrontata non con una successiva lettura del mondo, bensì con una semplificazione mentale che abbia isolato alcuni aspetti traducibili in numeri.

Precisamente, la rivoluzione si fonda su due pilastri.

1. Sensate esperienze (osservazioni della natura, resoconti di esperimen-

ti) compiute con i sensi potenziati da strumenti, come il telescopio e l'orologio.

2. Necessarie dimostrazioni: in un teorema, se concordiamo con le premesse e capiamo la dimostrazione, dobbiamo per forza accettare la conclusione.

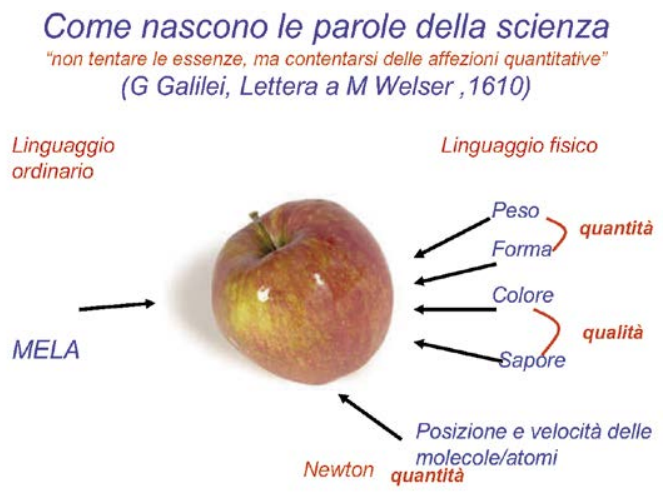

Figura 19 - In una lettera del 1610, Galileo enuncia il programma scientifico: «Non tentare le essenze, ma contentarsi delle affezioni quantitative».La mela diventa una collezione di dati misurati (come oggi il codice a barre di un supermercato). Le ambiguità delle parole con cui si designano le cose sono superate dall'insieme dei numeri ottenuti come valori delle misure.

Anche Francis Bacon propone sensate esperienze, registrate in tavole, e necessarie dimostrazioni utilizzando la logica, attraverso l'esclusione di alcune ipotesi. La differenza sta nell'uso della matematica: se l'ipotesi è espressa da una formula matematica, le conseguenze risultano semplicemente usando il formalismo (algoritmo).

La rivoluzione della scienza moderna supera le incertezze del prigioniero di Platone (Figg. 20 e 21); sembra estrarre dall'esperienza le sole cose che contano, le quantità da connettere con vincoli matematici, ben noti alla cultura dell'epoca perché elaborati da millenni. 
Visioni multiple ed elaborazione linguisticaRivoluzione di Galileo

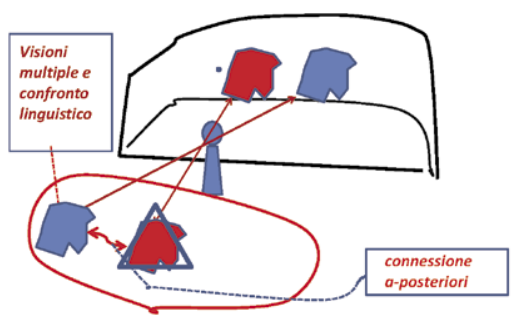

Rivoluzione Galileo=

scelta di un punto di vista che isola un nucleo matematico ("non tentare le essenze ma contentarsi delle affezioni quantitative...")

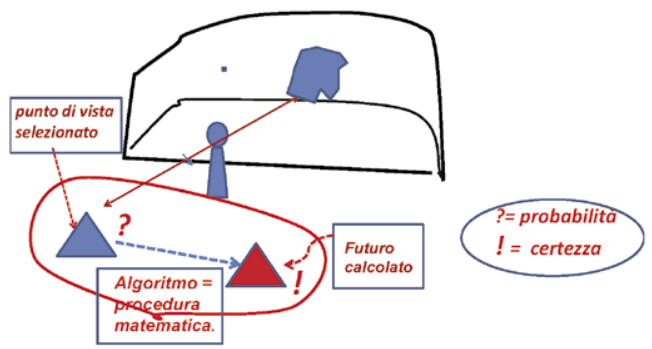

Figura 20 - a) Galileo opera una selezione linguistica che isola di ogni situazione aspetti quantitativi - corrispondenti a misure precise. b) Di conseguenza, l'algoritmo che rappresenta il Bayes inverso è ora una procedura matematica che assicura non probabilità ma certezze (vedi figura seguente).

\section{Da probabilità a certezza matematica}

Futuro in Bayes: dipende da $\mathrm{P}(\mathrm{d} \mid \mathrm{h})$

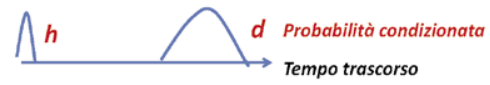

Futuro in Galileo-Newton:

$\mathrm{d}=$ funzione di h e del tempo $\mathrm{t}$ intercorso

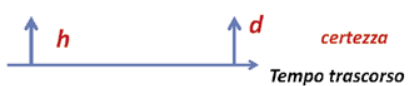

Figura 21 - Differenza fra probabilità e certezza; nel primo caso si ha un intervallo di valori pesati dalla funzione a campana; nel secondo caso risulta un solo valore. Una funzione 'peso' infinitamente stretta è detta delta di Dirac (vedi Parte C). 
Ogni cosa può essere vista come una particella dotata di proprietà espresse da numeri. A partire dal 1687, Newton introdusse le leggi che regolano il moto di una particella cioè la sua posizione nello spazio (visto come un continuo a 3 dimensioni) ad ogni istante (il tempo è visto come una quarta dimensione, orientata dal passato verso il futuro). Qualunque oggetto macroscopico è descritto dalle particelle che lo compongono.

Si credeva di avere con le equazioni di Newton una teoria universale, una 'teoria del tutto' - come si dice oggi - che cattura tutti gli aspetti rilevanti della realtà; da qui la presunzione deterministica di Laplace: «ad una intelligenza che conosca la posizione delle particelle a un certo istante, il futuro non riserva alcun mistero (determinismo)». In effetti, dopo Laplace si sono trovati dei limiti, alcuni elencati in figura 22.

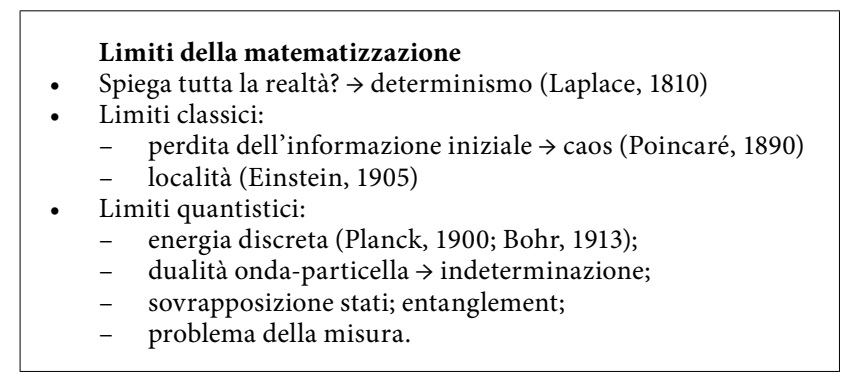

Figura 22 - La presunzione di Laplace e i limiti della matematizzazione. Limiti classici: i) il caos limita la predicibilità; ii) la relatività speciale impone la velocità della luce come velocità limite a cui trasmettere l'informazione. I limiti quantistici saranno esaminati nella Parte B.

Illustro di seguito il primo limite classico, data la sua rilevanza per le procedure cognitive che stiamo discutendo.

Schematizziamo (Fig. 23) con una retta (linea spessa) la traiettoria calcolata dalle leggi di Newton per una particella che all'istante iniziale sia nella posizione indicata dalla stella. Investighiamo la stabilità trasversale di ogni punto della traiettoria. Se il punto è stabile, sarà come sul fondo di una ciotola: una perturbazione viene corretta e il punto ricade sulla linea spessa, che pertanto è affidabile ad ogni futuro, come affermato da Laplace. Se invece la traiettoria è trasversalmente instabile, ogni punto è come sulla cima di un colle. Una minima incertezza iniziale viene amplificata nel corso del tempo e l'informazione relativa persa. Per il sistema solare, il tempo $\tau=1 / K$ entro cui si perde l'informazione iniziale è dell'ordine del milione di anni (perciò i moti planetari ci sembrano stabili), ma per un neurone della corteccia cerebrale è di pochi millisecondi, il che renderebbe precaria una percezione, per la cui esecuzione occorre una frazione di secondo (ad esempio, $200 \mathrm{msec}$ ). 


\section{Dinamica non lineare a 3 o più corpi}

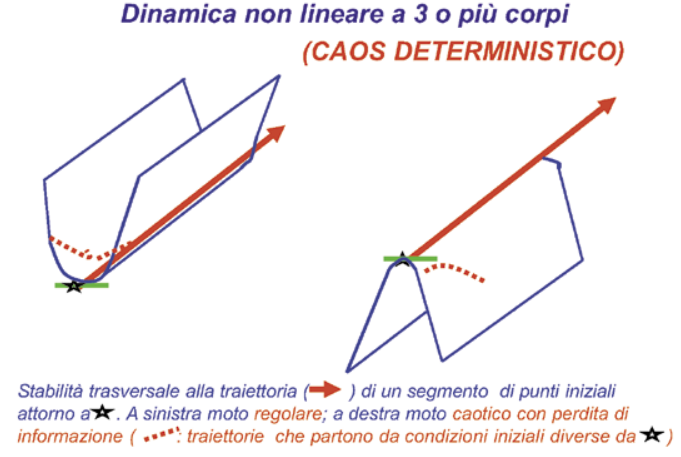

Figura 23 - Confronto fra traiettoria trasversalmente stabile e instabile. Nel primo caso ogni punto della linea rossa va visto come immerso in una ciotola (vedi Parte B) ed una incertezza nella condizione iniziale (segmento attorno alla stella)viene corretta al passare del tempo. Nel secondo caso, ogni punto della traiettoria è dal punto di vista trasversale sulla cima di un colle ed una minima incertezza iniziale viene amplificata al passare del tempo. L'informazione contenuta nel punto iniziale viene persa entro un tempo $\tau$ caratteristico, il cui reciproco $K=1 / \tau$ è chiamato entropia di Kolmogorov.

La figura 24 mostra come si controlla il caos aggiungendo variabili ulteriori al modello. Si ottiene così la riduzione o addirittura l'inversione della pendenza instabile. Nei processi percettivi, le interazioni collettive fra i neuroni della corteccia cerebrale correggono il caos del singolo neurone; ma se si esagera, si arriva a una percezione stabile, che rimane per sempre e impedisce la manipolazione di ulteriori stimoli esterni.

\section{Dinamica caotica: controllo(1990)}

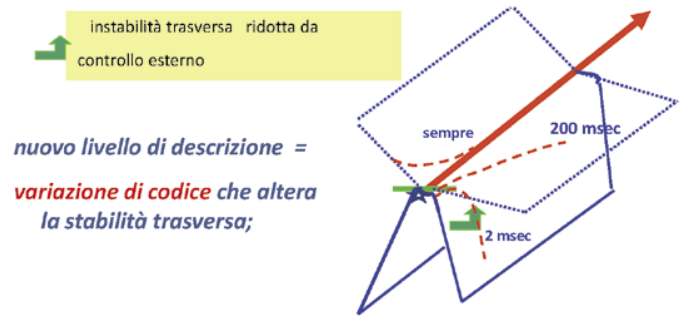

Percezione: affidabile se ha vita lunga ma non infinita!

Figura 24 - Controllo del caos: per aumentare il tempo $1 / \mathrm{K}$, occorre mitigare la pendenza della instabilità trasversale o addirittura invertirne il senso, immergendo la traiettoria in un potenziale stabile. Il controllo consiste nell'includere nel sistema dinamico ulteriori gradi di libertà che alterino la stabilità. Con riferimento ai tempi 
dei processi neuronali che generano la percezione, in figura si presenta l'originale potenziale instabile con due lembi (a destra e sinistra) che diano una perdita di informazione entro $2 \mathrm{msec}$; e due controlli che portino il tempo di perdita rispettivamente a $200 \mathrm{msec}$ (caratteristico della percezione) e all'infinito (situazione patologica che blocca ulteriori percezioni, perché ha un tempo di memoria $1 / \mathrm{K}$ infinito). Di questi due casi, si presenta in figura per chiarezza solo un lembo (a destra per 200 msec e a sinistra nel secondo caso).

Poincaré nel 1890 mostrò che quello che oggi chiamiamo 'caos deterministico' non è così esotico; il passaggio da stabilità a instabilità trasversale corrisponde al passaggio da un problema dinamico a due corpi (ad es. Terra-Sole) a quello a tre corpi (ad es. Luna-Terra-Sole). Dunque, fermarsi a Newton porta a situazioni patologiche.

Il progresso della scienza è legato a nuove osservazioni che non sono spiegate dalle teorie correnti e richiedono nuovi algoritmi interpretativi. In figura 25 si mostrano quattro esempi cospicui, descritti in dettaglio nella Parte B.

1. Nel Settecento, elettricità, magnetismo e ottica erano considerati come classi di fenomeni diversi, retti pertanto da leggi diverse. A metà Ottocento, Maxwell formulò una teoria unificata di questi fenomeni.

2. A fine Ottocento, Mendeleev raccoglieva tutti gli elementi chimici noti in una Tavola, che evidenziava le somiglianze e differenze. La teoria quantistica di Bohr (1913) e il principio di esclusione di Pauli (1925) permettevano di unificare in un unico schema tutta la sequenza di elementi.

3. Negli anni' 60 del secolo scorso, la fisica sub-nucleare aveva individuato un centinaio di particelle diverse, le cui masse non sembravano obbedire a particolari regolarità. L'ipotesi dei quarks di Gell-Mann (1964) permetteva di 'costruire' tutte le particelle; il campo di Higgs (ipotizzato da Higgs e altri a fine Novecento e osservato nel 2012) spiega i valori delle masse;

4. Le transizioni di fase (per esempio, la transizione da gas a liquido) passano per punti critici, al variare della pressione e temperatura a cui è soggetto il campione; in generale, punti critici sono osservati ogni volta che si passa da particelle indipendenti (come i sassi in un mucchio) ad un oggetto unificato la cui descrizione non richiede la conoscenza dei componenti. Attorno ai punti critici, valgono delle 'leggi di scala', nel senso che variando parametri esterni si può prevedere il nuovo comportamento. Le transizioni di fase riguardano qualunque sistema composto da ingredienti elementari (ad esempio, i singoli magnetini atomici di una calamita) che passa ad un comportamento globale (nell'esempio, tutti i magnetini paralleli) descrivibile senza far intervenire i componenti. La teoria delle transizioni di fase ha trovato una spiegazione universale nel cosiddetto 'gruppo di ri-normalizzazione' di Wilson (attorno al 1970). 


\begin{tabular}{|ll|}
\hline \multicolumn{2}{|c|}{ Comprensione della realtà: quattro esempi } \\
\hline 1. Elettricità-magnetismo-ottica & Equazioni elettromagnetismo (Maxwell) \\
\hline 2. Tavola di Mendeleev & Atomo quantistico (Bohr, Pauli) \\
\hline $\begin{array}{l}\text { 3. Zoo di 100 particelle elementari con } \\
\text { masse differenti }\end{array}$ & $\begin{array}{l}\text { Quarks (M Gell Mann) e campo di Higgs } \\
\text { che fissa le masse }\end{array}$ \\
\hline 4. Leggi di scala in transizioni di fase & Gruppo di ri-normalizzazione (K. Wilson \\
\hline
\end{tabular}

Figura 25 - Quattro esempi di 'salto di paradigma', cioè di fuoriuscita dalla scienza normale (bayesiana) per introdurre nuovi formalismi, corrispondenti a 'salti di algoritmo'.

\section{Il ruolo dei processi linguistici nei programmi scientifici}

Il programma galileiano è consistito nel 'non tentare le essenze' (cioè non attribuire un valore di realtà a quanto osservato) ma ridurre l'osservato a una collezione di numeri (estratti dai vari dispositivi di misura): un po' come il codice a barre di un oggetto al supermercato. La fisica credeva di potersi limitare a connettere questi numeri mediante algoritmi (teorie scientifiche), escludendo qualunque ontologia, cioè conoscenza della 'cosa in sé' per dirla con Kant.

Le considerazioni linguistiche sopra presentate ridanno un ruolo all'ontologia come recupero delle 'essenze'.

In Bayes diretto, l'algoritmo $P(d \mid h)$ è pre-assegnato come l'istruzione a un computer (replicatore fisso: gene nella biologia, meme nell'evoluzione culturale) modificabile solo per mutazione casuale. Invece, in Bayes inverso, il programma esecutivo $P(d \mid h)$ nasce dal confronto fra $d$ e i brani $h^{*}$ del testo che hanno preceduto $d$.

Questa costruzione a posteriori indica una adaequatio intellectus et $r e i$, cioè la selezione della 'risposta' $P(d \mid h)$ più opportuna con cui la 'cosa' si presenta da un certo versante $h^{\star}$. Il programma linguistico della scienza descrive dunque la cosa e non più l'oggetto misurato (cioè il corrispondente codice a barre) del programma riduzionistico; ritorna l'ontologia che sembrava esclusa dal programma scientifico.

In base a quanto detto si può fare la seguente obiezione alla Critica della ragion pura di Kant: i trascendentali di Kant sono programmi $P(d \mid h)$ che scelgono l' $h^{\star}$ per ogni input $d$; precedono il confronto $h^{*}-d$, invece di esserne la conseguenza.

La figura 26 mostra come si passa da una teoria ingenua, che procede appplicando lo stesso algoritmo a situazioni semanticamente più ricche e pertanto implicando una complessità algoritmica $C$ (lunghezza del programma) e una entropia di Kolmogorov $K$ (perdita di informazione per 
caos deterministico) crescenti ad una teoria efficace che, utilizzando elaborazioni linguistiche, porti a bassi $\mathrm{C} \mathrm{e} \mathrm{K}$.

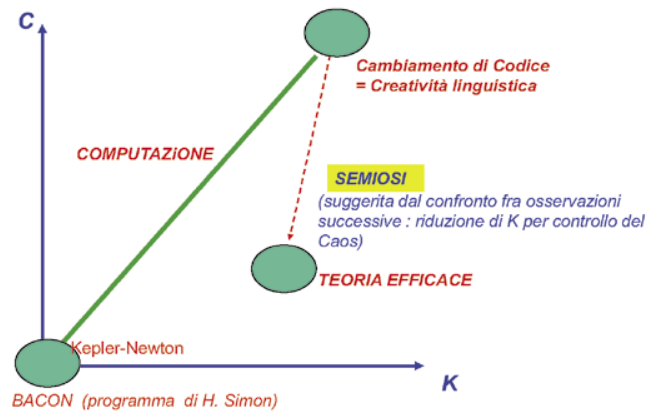

Figura 26 - Diagramma C (complessità algoritmica = lunghezza in bit del programma di calcolo) su asse verticale e K (entropia di Kolmogorov = inverso del tempo di perdita dell'informazione per Caos deterministico) su asse orizzontale. In una teoria scientifica con algoritmi prestabiliti, $\mathrm{C}$ e $\mathrm{K}$ crescono da valori piccoli (come nel programma BACON di $\mathrm{H}$. Simon che valuta le orbite newtoniane, come esempio del potere dell'intelligenza artificiale) a valori sempre più alti per situazioni non così semplici come le orbite planetarie. In tal caso, una atto di semiosi (riconoscimento linguistico di altre variabili cruciali) porta a ridurre $\mathrm{C}$ e $\mathrm{K}$ e dà una teoria 'efficace,' con bassi $\mathrm{C}$ e $\mathrm{K}$.

Prendiamo ad esempio la biologia: l'epigenetica dice che l'attività di un gene non è fissata una volta per tutte come l'algoritmo in Bayes diretto, ma è regolata dal confronto fra il pacchetto $h^{\star}$ delle competenze acquisite e l'ultimo invasore $d$ (virus o qualunque altra perturbazione ambientale); dunque emerge un $P(d \mid h)$ come nel Bayes inverso.

Oggi si parla di Emergenza o Anti-riduzionismo. In fisica, il dibattito fu aperto nel 1972 dal lavoro More is different (un articolo di P.W. Anderson). Riassumiamo le considerazioni sul ruolo del linguaggio nel programma scientifico, ribadendo che la fisica non è chiusa dal punto di vista informazionale. Precisamente:

1. caos: implica perdita di informazione;

2. controllo del caos: implica ri-codifica = cambio della descrizione forma$l e=$ scambio di informazione. Come nella traduzione di un testo, vedi al riguardo Frye (1981), in cui si dibatte il problema della traduzione della Bibbia dall'Ebraico (5000 vocaboli) a una lingua moderna (100 volte più vocaboli).

3. ri-codifica: si basa su un dialogo con l'ambiente = semiosi;

4. la ri-codifica non è algoritmica; l'intelligenza non è problem solving come ritenuto da Herbert Simon, padre dell'intelligenza artificiale e autore del programma BACON citato in figura 26. 
Il ruolo del linguaggio nel programma scientifico è racchiuso in tre parole chiave, illustrate in un piccolo libro (Arecchi 2007):

- coerenza = ordine su scale grandi (a lungo raggio): nello spazio (visione) o nel tempo (musica);

- complessità (semantica $)=$ coesistenza di differenti domini di coerenza (i colli di figura 16): da non confondere con complessità algoritmica $=$ lunghezza in bit di un algoritmo;

- creatività = salto da un dominio di coerenza a un altro.

Concludiamo queste considerazioni presentando il limite dei salti nonalgoritmici, cioè delle nuove teorie senza adeguato supporto sperimentale (si veda al riguardo lo schema di Fig. 27).

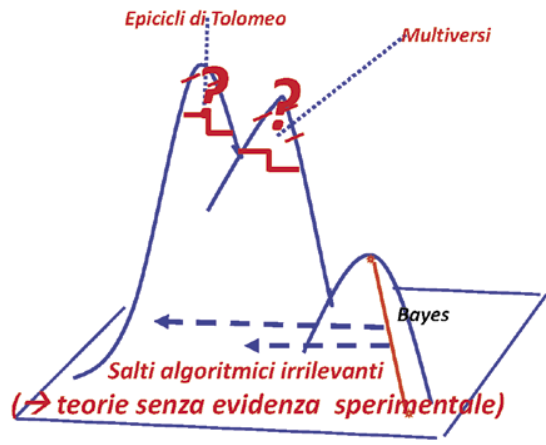

Figura 27 -Attenti alle trappole linguistiche, che 'inventano' teorie senza un adeguato supporto sperimentale. Tali sono la teoria degli epicicli di Tolomeo e l'attuale congettura dei multiversi (pluralità di universi, ma senza possibilità di mutua comunicazione). Il pericolo è discusso da Ellis e Silk (2014).

\section{Le due ermeneutiche: circolo e spira}

L'aspetto creativo dell'attività linguistica ne rappresenta anche il pericolo. Se a partire da un ente immaginato se ne estraggono tutte le potenzialità, si corre il rischio di creare delle Chimere, nelle quali coesistono aspetti in mutuo contrasto. Tale è lo status di tutti i prodotti della creazione poetica (Fig. 28). È come se il Poeta, una volta attribuite certe capacità al suo personaggio, si lasciasse coinvolgere da trappole dinamiche quali il caos, che distrugge alcuni aspetti e li rimpiazza con altri.

Supponiamo che $A$ sia un osservatore e $B$ un osservato. La differenza fra una scienza normale - che continua ad applicare lo stesso algoritmo - e la scienza dei confronti linguistici, che opera con salti di algoritmo, 
corrisponde allo scontro fra due ermeneutiche, cioè di due letture interpretative del mondo, rappresentate rispettivamente con un circolo e una spira (Fig. 29).

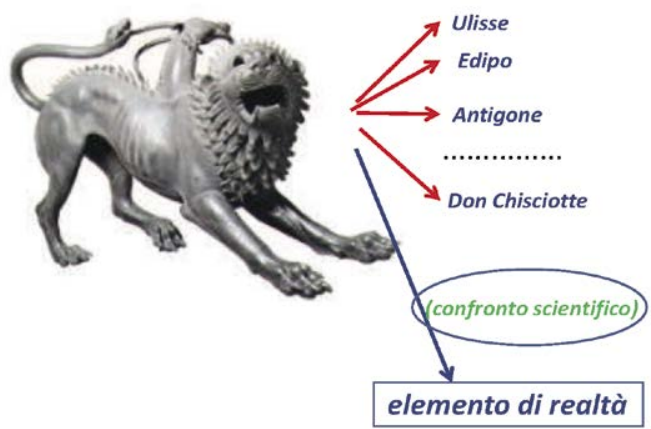

Figura 28 - Due aspetti della creatività linguistica. I protagonisti di una creazione artistica (da Ulisse a Don Chisciotte), una volta caratterizzati da certi comportamenti, possono sviluppare situazioni contrastanti, presentando pertanto aspetti ambigui, come la Chimera di Arezzo. Se invece l'attività linguistica sta confrontando sequenze di osservazioni, emergono elementi di realtà, che risolvono le ambiguità.

Nel circolo, $A$ applica un algoritmo e crede di aver estratto gli aspetti rilevanti di $B$; ogni volta che torna ad osservare $B$, ne estrae gli stessi aspetti e conferma se stesso come $A$ (fondamentalismo). Nella spira, $A=$ $A 1$ è consapevole che una singola osservazione gli presenti una prima approssimazione $B 1$ di $B$; ripetendo l'osservazione ma sotto un altro versante (l'attività linguistica permette questa pluralità di prospettive), $A 1$ coglie un aspetto più ricco $B 2$ ed evolve in $A 2$, e così via in una sequenza di scambi $A-B$ che abbiamo figurato come una spira (dialogo senza fine). Si corregge in tal modo il limite del prigioniero di Platone, in quanto abbiamo la possibilità di variare il punto di vista. Dunque, non si cattura la realtà una volta per tutte, ma si instaura un dialogo senza fine.

Il solipsimo idealistico aveva ipotizzato una crescita del soggetto linguistico in assenza di un riferimento esterno $B$ (Fig. 30). Tale ipotesi può spiegare l'insorgere di Chimere nella creazione estetica, ma è priva di consistenza scientifica.

Si noti che la spira spiega il rapporto fra persone, che un'ermeneutica ripetitiva credeva di congelare in una singola osservazione; quando $A$ e $B$ sono entrambi persone, allora la spira è simmetrica: come $A$ si modifica $(A 1,2,3 \ldots)$ cogliendo dettagli sempre più precisi di $B$, così la sequenza $B 1,2,3 \ldots$ non denota solo gli incrementi di conoscenza in $A$, ma l'attività propria di $B$ che 'cresce' nel rapporto con $\mathrm{A}$. Dunque, definiamo 'persone' due soggetti linguistici in mutua interazione, cioè in dialogo senza fine. 


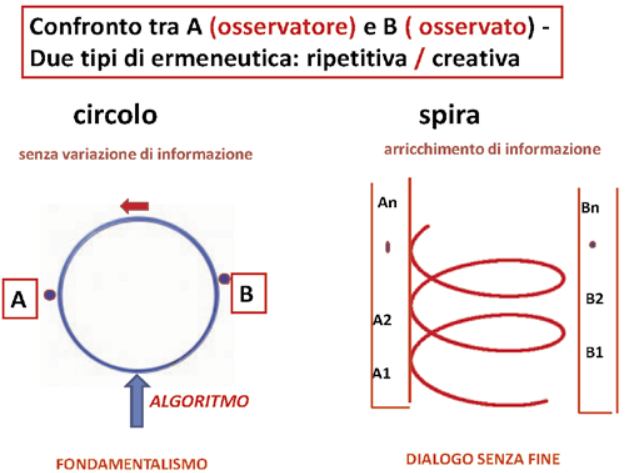

Figura 29 - L'ermeneutica ripetitiva del Bayes diretto che sfrutta lo stesso algoritmo (circolo) e l'ermeneutica creativa del confronto linguistico fra osservazioni successive, con la scoperta di nuovi aspetti codificati da Bayes inverso (spira).

Nell'ermeneutica creativa, c'è il confronto tra $A$ e $B$, dove $B$ è la realtà esterna che limita e guida le scelte successive di $A$. Invece, nella dialettica di Hegele, lo Spirito evolve ma senza un referente esterno; lo stesso nel 'pensiero debole'. Limitandoci all'attività estetica, l'assenza di referente esterno è la base delle Chimere.

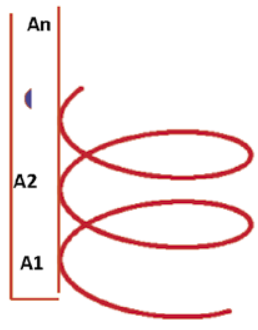

Manca il feedback, cioè il dialogo con un altro. Si passa da una ontologia con referente esterno (realistica) ad una ontologia solipsistica

Figura 30 - Il punto di vista del solipsismo idealistico: $A$ cresce per propria attività senza avere un referente esterno.

La stessa procedura creativa (= generatrice di novità) e senza fine della Spira può essere ritrovata in:

i) apprezzamento estetico (dialogo con un testo linguistico);

ii) investigazione scientifica (dialogo con un ente di natura);

iii) vita d'amore (dialogo fra persone);

iv) vita oltre la morte (vita mutatur, non tollitur). 
Come in i) dietro l'opera d'arte con cui si instaura un dialogo senza fine c'è un creatore, così in ii) dietro ogni evento c'è il Creatore che è Logos. Come in iii) la persona instaura un dialogo con un'altra persona, così in iv) si può credere senza cadere in contraddizioni in un dialogo eterno con il Logos. L'ermeneutica a spira offre dunque una base per un approccio alla teologia cristiana del Verbo.

Vediamo come la semiosi implicata dall'ermeneutica a spira offra una base per giustificare la libertà umana.

Per quanto detto, siamo a rigori dentro un programma deterministico, ma a partire da un sistema che non è quello che percepiamo (come il prigioniero platonico) ma quello che ri-codifichiamo, aggiungendo le variabili che controllano il caos; abbiamo introdotto una stabilizzazione omeo-dinamica in misura maggiore o minore (cooptando variabili aggiuntive dalla memoria) a seconda che si stabilizzi il caos per un secondo o un giorno. Questa ambiguità nel risultato è compatibile con le leggi fisiche: essa è la base della creatività scientifica ma anche della libertà etica.

$\grave{E}$ per questo che ci sono sfumature varie fra genio e cretino, fra santo e peccatore incallito: tutte categorie che non valgono per la macchina di Turing. É perciò che - a seguire Newton ma non Poincaré - si introduce (vedi Kant) una barriera fra ragion pura (regno del determinismo) e ragion pratica (regno della libertà).

In conclusione, il Bayes inverso ci permette di affrontare rigorosamente questioni che per i fondamentalisti (cultori dell'ermeneutica a circolo) fanno parte delle credenze private, senza validità scientifica. Ricordo al riguardo una lettura liceale di Benedetto Croce, in cui il filosofo criticava l'idea di vita eterna in base alla seguente considerazione: in una comunità di persone, dopo un po' si sa tutto uno dell'altro, e si passerebbe l'eternità ad annoiarsi. Due risposte, sulla base di quanto visto finora:

1. nel dialogo senza fine, l'informazione continua a crescere, perché $A$ e $B$, nel dialogo d'amore, continuano a modificarsi reciprocamente;

2. in che cosa consiste lo scorrere del tempo? Se siamo calati nelle cose di tutti i giorni, il loro cambiare misura il tempo che passa e la scienza può legittimamente porsi domande sulla fine di tutto; ma se siamo impegnati in un dialogo tra persone, il tempo è scandito dal progressiva mutua trasformazione (A1-B1-A2-B2-ecc.) e la procedura può continuare senza fine.

Senza la pretesa di approfondire temi filosofici, ritengo corretto dire che quanto detto finora fornisce una base per una ontologia come recupero delle 'essenze'.

Riassumiamo, infine, cose già dette.

Nella percezione, $P(d \mid h)$ è pre-assegnato come l'istruzione a un computer. Invece, nel giudizio, il programma esecutivo $P(d \mid h)$ nasce dal confronto fra $d$ e i brani $h^{*}$ del testo che hanno preceduto $d$. Questa costruzione indica una conformità dell'intelletto alla cosa (adaequatio intellectus et $r e i)$, cioè la selezione della 'risposta' $P(d \mid h)$ più opportuna con cui la 'cosa' 
va letta da un certo versante. È la cosa e non più l'oggetto (= codice a barre) del programma riduzionistico.

Al riguardo, come già detto, i trascendentali di Kant sono programmi $P(d \mid h)$ che scelgono l' $h^{*}$ per ogni input $d$. Critica: precedono il confronto $h^{*}$ $d$, invece di esserne conseguenza, come invece ha luogo nel Bayes inverso.

\section{Cognizione e Realtà: domande e risposte}

\section{DomANDA 1 - Poteri e LIMITI DEL LINGUAGgio SCIENTIFICO.}

\section{Risposta 1 -Sistemi aperti, Complessità}

L'emergenza di nuovi stati implica che stiamo osservando sistemi aperti, che scambiano energia ed informazione con l'ambiente. Tali sono i sistemi viventi, i sistemi sociali. Li chiamiamo complessi. Con ciò intendiamo che la loro descrizione non è racchiudibile in un algoritmo. Nei sistemi chiusi, possiamo specificare tutte le caratteristiche, e la complessità è legata alle difficoltà di risoluzione del problema, è complessità epistemica. Per un sistema aperto, ogni modello è una proiezione da uno spazio a molte dimensioni a un sotto-spazio a numero accessibile di pochi gradi di libertà, quelli che siamo riusciti a catturare nel modello (in figura 20 chiamato teoria efficace).

Euristicamente, è come catturare su una telecamera gli aspetti salienti di un paesaggio: se c'è un riferimento vistoso, esso caratterizzerà il paesaggio, ma in genere dobbiamo collezionare diversi punti di vista: ognuno è vero ma di una verità parziale. Chiamiamo allora complessità di un sistema aperto la molteplicità di modelli rilevanti che riusciamo a formulare, essa è legata all'ontologia del problema (vedi Risposta 2); la designeremo come complessità ontica (cfr. il paesaggio a molti colli di Fig. 8).

DOMANDA 2 - DESCRIZIONE SCIENTIFICA = MAPPA UNO-A-UNO DELLA REALTÀ?

o piuttosto

Domanda 3 - STRUMENTO CONVENZIONALE, E PERTANTO LA REALTÀ SFUGGE ALLA SCIENZA?

\section{Risposta 2 -Ontologia dei livelli scientifici, Analogia}

La dinamica non lineare introduce differenze qualitative che caratterizzano livelli di realtà $=$ scienze diverse. Non è un espediente linguistico, per cui in definitiva si possa ridurre tutto a componenti fondamentali. Dobbiamo prendere atto di una consistenza ontologica delle entità che compaiono a un certo livello. L'emergenza di queste entità è caratterizzata da biforcazioni (le catastrofi di R. Thom).

Esempi di emergenza sono il campo coerente laser, la rigidità di un oggetto metallico, l'insorgere della vita e del linguaggio simbolico. Poiché ogni descrizione scientifica è proiezione basso-dimensionale di una situa- 
zione alto-dimensionale, descrizioni diverse, o linguaggi, sono appropriate per lo stesso stato di cose. In questi linguaggi la stessa parola può essere utilizzata, ma con connotazioni diverse che rappresentano diversi punti di vista. Tuttavia le diverse connotazioni non sono del tutto prive di correlazioni in quanto si riferiscono allo stesso pezzo di realtà.

Questa correlazione semantica è stata chiamata analogia.

\section{Risposta 3 -Contingenza}

Ogni livello scientifico rappresenta una verità parziale, ma esso deve essere aperto ad altri livelli di conoscenza.

Verità parziale significa che il livello considerato si riferisce a qualcosa che esiste, ma questa esistenza è contingente. La cosa potrebbe anche non esistere. La fisica moderna è stata tentata di assegnare un valore di necessità ad enti fondamentali, ma tale necessità è solo logica.

Ogni particella può trasformarsi in altre, cioè cessare di esistere in quanto tale. Nasce pertanto la domanda: chi è il necessario supporto per gli esseri contingenti? In effetti la critica dell'argomento della contingenza era basata sulla presunta incapacità delle descrizioni del mondo a entrare nell'ontologia (Hume e Kant).

L'analisi delle procedure scientifiche nelle Risposte 1 e 2 ha mostrato come invece sia legittimo che il discorso scientifico affronti l'ontologia.

DOMANDA 4 - LE DESCRIZIONI SCIENTIFICHE CATTURANO ASPETTI SALIENTI DELLA REALTÀ (CONTRO DOMANDA 3), MA NON LI ESAURISCONO (CONTRO DOMANDA 2), LASCIANDO SPAZIO PER ALTRE DESCRIZIONI RILEVANTI?

\section{Risposta 4 - I limiti di una apologetica settoriale}

Non presentare una apologetica basata su 'dimostrazioni scientifiche di Dio' e invece sottolineare:

- l'incapacità di ogni linguaggio scientifico a esaurire la realtà e

- tuttavia il fatto che la validità di descrizioni scientifiche settoriali implica che esse debbano avere una base ontologica.

Argomenti pro e contro sono basati su considerazioni:

- cosmologiche (J. Barrow, S. Hawking, S. Weinberg, S. Carroll);

- biologiche (J. Monod, R. Dawkins);

- di scienze cognitive (P. Churchland, D. Dennett).

Limite degli approcci settoriali: trascurano una analisi critica degli strumenti linguistici a disposizione della scienza, nello spirito di L. Wittgenstein.

Mio punto di vista: il cuore di un'apologia è rappresentato da una analisi del linguaggio scientifico, che mostri come esso debba essere considerato un sistema aperto (vedi Risposta 1). Questa apertura (ogni linguaggio è intenzionale, punta sempre a qualcosa) supera la chiusura di una analisi circolare del linguaggio considerato soltanto come un lessico più una grammatica, cioè un insieme finito di parole e di regole che connettono le parole. 
DOMANDA 5 - LIBERO ARBITRIO, LIBET: PIANIFICARE UN'AZIONE = DECISIONE ETICA?

Risposta 5 - Esperimenti di Libet; decisioni etiche implicano linguaggio, dunque la coscienza

Gli esperimenti (dal 1985) di Benjamin Libet indicano che l'esperienza soggettiva della volontà è successiva a una scelta già compiuta a livello pre-cosciente. Prima che in noi nasca consapevole il desiderio di compiere liberamente un movimento qualsiasi, il nostro cervello ha già attivato i processi necessari al compimento del movimento.

Nell'esperimento, il soggetto riceve l'indicazione di compiere un atto motorio, segnando l'istante in cui decide di farlo. Durante l'esperimento vengono registrati i potenziali corticali indicatori della prontezza all'azione motoria; tali indicatori precedono di circa $0,5 \mathrm{sec}$ l'esperienza cosciente.

Sembra dunque che l'inizio neurale inconscio dell'azione si mantiene tale per circa $0,35-0,4 \mathrm{sec}$, mentre il soggetto diviene consapevole della sua intenzione di agire solo 0,15-0,2 sec prima che l'atto motorio abbia luogo.

Conclusioni dell'esperimento di Libet:

1. anche i movimenti volontari che riteniamo più spontanei hanno inizio da un'attività neurale inconscia;

2. la consapevolezza dell'intenzione di agire è successiva a questo inizio neurale inconscio di $0,34-0,4 \mathrm{sec}$;

3. il libero arbitrio va ripensato nel ruolo di semplice supervisore dell'azione.

Se ora invece - con Lonergan - consideriamo che una decisione sia frutto di un confronto linguistico fra due opzioni, presentate come due brani distinti a distanza di $3 \mathrm{sec}$, vediamo che la decisione etica implica un linguaggio, e non è il mero risultato di automatismi percettivi. Siccome un NCC ha luogo attorno a $1 \mathrm{sec}$, sulla scala dei $3 \mathrm{sec}$ non opera NCC, ma quella consapevolezza di se stessi che abbiamo chiamato coscienza.

\section{DOMANDA 6 - AI (INTELLIGENZA ARTIFICIALE) E MACHINE LEARNING: POTERI E LIMITI?}

Risposta 6 - AI (intelligenza artificiale) e machine learning: poterie limiti

La AI studia i fondamenti teorici, le metodologie e le tecniche che consentono la progettazione di sistemi hardware e software capaci di fornire all'elaboratore elettronico prestazioni che, a un osservatore comune, sembrerebbero essere di pertinenza esclusiva dell'intelligenza umana.

Inizialmente i ricercatori si concentrarono sullo sviluppo di algoritmi che imitassero fedelmente i ragionamenti degli esseri umani per realizzare deduzioni logiche in modo da poterli integrare all'interno dei sistemi intelligenti. Tali algoritmi si basano su una rappresentazione simbolica dello stato del mondo e cercano sequenze di azioni che raggiungano uno stato desiderato. 
La rappresentazione della conoscenza e l'ingegneria della conoscenza costituiscono contributi centrali per la ricerca nell'ambito dell'intelligenza artificiale. Fra le cose che un sistema intelligente ha la necessità di rappresentare troviamo frequentemente: oggetti, proprietà, categorie e relazioni fra oggetti, situazioni, eventi, stati, tempo, cause ed effetti, conoscenza posseduta da altri. La conoscenza e la sua rappresentazione sono cruciali soprattutto per quella categoria di sistemi intelligenti che basano il loro comportamento su una estensiva rappresentazione esplicita della conoscenza dell'ambiente in cui operano.

\section{Elaborazione del linguaggio naturale}

La capacità di elaborare il linguaggio naturale fornisce ai sistemi intelligenti la possibilità di leggere e capire il linguaggio utilizzato dagli esseri umani. Questa capacità si dimostra essenziale in tutte le applicazioni dell'AI che richiedano la ricerca di informazioni, la risposta a domande, la traduzione o l'analisi di testi. La difficoltà principale è l'intrinseca ambiguità che caratterizza i linguaggi naturali; per questo motivo le soluzioni richiedono un'estesa conoscenza del mondo.

\section{Movimento e manipolazione}

La robotica è correlata all'AI: i robot sono sistemi intelligenti per quei compiti che richiedono capacità di livello cognitivo per la manipolazione $\mathrm{o}$ lo spostamento di oggetti, con i sotto-problemi della localizzazione (determinare la posizione), della costruzione di mappe (apprendere le caratteristiche dello spazio circostante) e della pianificazione ed esecuzione dei movimenti.

Un agente è un'entità in grado di percepire l'ambiente attraverso sensori e di agire sull'ambiente attraverso attuatori. Ogni agente è quindi associato ad una sequenza di percezioni e ad una funzione agente, che specifica il comportamento associando ad ogni sequenza di percezioni un'azione da compiere.

Machine learning (apprendimento automatico) è un sottoinsieme dell'AI in cui il software analizza i dati, ne riconosce le caratteristiche e 'impara' dal loro esame. La macchina può così stabilire legami fra i dati e fare delle predizioni senza essere espressamente programmata per compiere queste attività. Il machine learning viene usato in quegli ambiti e in quelle applicazioni pratiche in cui progettare e programmare algoritmi espliciti è impraticabile: la macchina riesce quindi a costruire in modo induttivo un modello basato su dei campioni riuscendo così anche a prendere delle 'decisioni'.

Riguardo all'AI esistono due modi diversi in cui essa può essere intesa.

1. Intelligenza artificiale debole (weak $A I$ ): una macchina può essere programmata con delle regole ben definite, in modo da comportarsi in modo intelligente.

2. Intelligenza artificiale forte (strong AI): una macchina che agisce in modo intelligente implica che essa sia anche cosciente di come realmente si comporta. 
Nel 1950 Alan Turing (Turing 1950) porta il dibattito filosofico ad un livello pragmatico, partendo dal 'gioco dell'imitazione', a tre partecipanti: un uomo A, una donna $\mathrm{B}$, e una terza persona $\mathrm{C}$. Quest'ultimo è tenuto separato dagli altri due e mediante una serie di domande deve stabilire qual è l'uomo e quale la donna. Dal canto loro anche A e B hanno dei compiti: A deve ingannare $C$ e portarlo a fare un'identificazione errata, mentre $B$ deve aiutarlo. Affinché $\mathrm{C}$ non possa disporre di alcun indizio (come l'analisi della grafia o della voce), le risposte alle domande di $\mathrm{C}$ devono essere tele-trasmesse.

Il test di Turing si basa sul presupposto che una macchina si sostituisca ad A. Se la percentuale di volte in cui C indovina chi sia l'uomo e chi la donna è simile prima e dopo la sostituzione di A con la macchina, allora la macchina stessa dovrebbe essere considerata intelligente, dal momento che - in questa situazione - sarebbe indistinguibile da un essere umano.

Il test di Turing è dunque un gioco dell'imitazione nel quale un computer, adeguatamente programmato, prende il posto del giocatore A. La domanda, allora, non sarà più se le macchine sono in grado di pensare, ma: «Che accade se una macchina prende il posto di A?»; «Il giocatore $\mathrm{C}$ si sbaglierà altrettanto spesso nello stabilire il sesso di $\mathrm{X} \mathrm{e} \mathrm{Y} \mathrm{come} \mathrm{quando} \mathrm{al}$ gioco dell'imitazione prendono parte un uomo e una donna?».Il risultato di questa riformulazione del problema sta nel fatto che «viene tirata una linea di separazione abbastanza netta tra le capacità fisiche e quelle intellettuali di un uomo». Tale idea contribuisce a dare una connotazione del concetto di 'intelligenza' fortemente distaccata dalle caratteristiche fisiche, quasi che queste siano affatto trascurabili. Secondo Turing, se una macchina fosse in grado di ingannare sistematicamente gli interroganti, essa mostrerebbe un comportamento totalmente indistinguibile da quello di un essere umano.

Il filosofo John Searle ha proposto una modifica al test di Turing, che ha preso il nome di stanza cinese, sostenendo l'inattendibilità del test di Turing. L'argomentazione della stanza cinese è la seguente. Si supponga che un computer si comporti come se capisse il cinese. Il computer prenderebbe dei simboli cinesi in ingresso, eseguirebbe un programma e produrrebbe altri simboli cinesi in uscita. Il comportamento superi il test di Turing. In altre parole, il computer possa convincere un cinese di parlare con un altro uomo che parla correttamente cinese, mentre in realtà sta parlando con un calcolatore. A tutte le domande dell'umano il computer risponderebbe appropriatamente. I sostenitori dell'AI forte concludono che il computer capisce la lingua cinese, come farebbe una persona, in quanto non c'è nessuna differenza tra il comportamento della macchina e di un uomo che conosce il cinese.

Ora, Searle si immagina in una stanza (la stanza cinese) con un libro contenente la versione in inglese del programma utilizzato dal computer e carta e penna. Searle potrebbe ricevere scritte in cinese, elaborarle seguendo le istruzioni del programma, e produrre altri simboli cinesi in uscita, come faceva il calcolatore. Egli non capisce i simboli cinesi. Quindi la sua mancanza di comprensione dimostra che il calcolatore non può comprendere il cinese, poiché esso è nella sua stessa situazione. Il calcolatore è un 
semplice manipolatore di simboli, esattamente come lo è lui nella stanza cinese - e quindi i calcolatori non capiscono quello che stanno dicendo tanto quanto lui. L'uomo crea l'apparenza della comprensione del cinese seguendo le istruzioni di manipolazione dei simboli, ma non giunge per questo a capire il cinese. Poiché un computer fa lo stesso - manipolare simboli in base alla sola sintassi - nessun computer, semplicemente eseguendo un programma, giunge a comprendere realmente il cinese.

Il punto di Searle è la distinzione tra sintassi e semantica. La stanza è in grado di combinare i caratteri secondo le regole, ma essa non conosce il significato di ciò che ha fatto, cioè non ha contenuto semantico. I caratteri non rappresentano neppure simboli perché non sono interpretati in nessuna fase del processo.

Riprendendo e ampliando la Risposta: in base a quanto abbiamo discusso sul linguaggio, il confronto umano fra due brani di un testo, per elaborare il Bayes inverso, implica (vedi ermeneutica a spira) quello che W. von Humboldt ha chiamato "uso infinito di risorse finite»; fra due successive ri-presentazioni, l'uomo 'ha vissuto', cioè - essendo immerso nel mondo - si è riconfigurato negli apprezzamenti e valutazioni, e di questo porta traccia in quella presenza di sé a se stesso che abbiamo chiamato coscienza. Come ha detto G. Bateson, il nostro conoscere è ecologico.

Niente di tutto questo è implicato nelle operazioni di una macchina di calcolo; pertanto l'AI non può arrivare alla creatività dell'ermeneutica a spira, anche se è un aiuto potentissimo per velocizzare e ottimizzare l'ermeneutica a circolo.

\section{Teoria matematica del linguaggio}

Il linguaggio visto come corpo lessicale e insieme di regole grammaticali (natural language) è stato formulato da N. Chomsky. Nella misura in cui è risolubile da un corpus di algoritmi, non implica il confronto che porta al Bayes inverso. Riferendoci a figura 9, siamo nell'ambito delle teorie decidibili da un algoritmo, più o meno complesso. Qui l'AI può introdurre vantaggi enormi, ma non è questo il linguaggio creativo introdotto e discusso a partire da figura 8 e che porta alla risoluzione del mito platonico di figura 12 e all'ermeneutica a spira (vedi Fig. 29).

Analoghe considerazioni valgono per qualunque decisione frutto di un giudizio.

Prendiamo ad esempio il tema della bellezza. È stata associata alle spinte riproduttive (è bello quello che stimola sessualmente) e su questa linea, che sembra spiegare l'arte classica, sono state sviluppate considerazioni darwiniane. Se fosse così, si parlerebbe di bellezza per Prassitele ma non per Picasso (si veda ad esempio Lesdemoiselles d'Avignon). Ma se invece la bellezza emerge dal confronto armonico fra due brani testuali successivi, come discusso nel Bayes inverso, allora vien meno l'equivoco 'naturalistico' e si cercheranno evidenze di congruenza interna a una opera. Lo stesso valga in musica nel confronto fra Mozart e A. Schoenberg. 
Domanda 7 - Oltre Al Linguaggio SCIENTIFico, Ci SONO ALTRI LINGUAGGI, CHE DICONO COSE SENSATE SULLA REALTÀ?

Risposta 7 - Il programma scientifico non può essere demandato ad AI

La nascita del programma scientifico è squisitamente linguistica: in figura 20 a), l'estrazione di quantità suscettibili di trattazione matematica (si veda la mela di figura 19) è un atto di creatività linguistica. Un AI può svolgere con successo e meglio di un operatore umano tutte le successive operazioni matematiche, ma non può attivare questo primo passo.

Il cuore del Bayes inverso su cui si fonda il linguaggio è stato illustrato in figura 13 come confronto fra due brani successivi di un testo. Cruciali sono le connotazioni che attribuiamo ai due brani; queste connotazioni sono legate al nostro vissuto umano, sono (per dirla con G. Bateman) 'ecologiche'.

È per questo che il ritorno dopo anni a un testo (letterario, musicale, figurativo) porta a punti di vista inediti perché nell'attore linguistico si è accumulata tanta esperienza ulteriore. Invece, l'AI non ha questa virtù ecologica, gli manca il passo linguistico cruciale di figura 20 a): non sa quali entità matematiche astrarre per immetterle in un programma.

In figura 26 si menziona il programma BACON di H. Simon, che esegue il calcolo delle orbite newtoniane; ma il trasformare i corpi celesti in numeri è un atto linguistico che precede l'intervento di AI.

Per rispondere alla Domanda 5 sui linguaggi non scientifici ritengo che la speculazione metafisica aristotelica, con la distinzione fra potenza e atto e con l'elencazione della quattro cause (materiale, efficiente, formale e finale), sia un programma linguistico che estrae aspetti sensati del mondo, anche se non è fatta per costruire previsioni. Il programma linguistico della scienza descrive dunque la cosa e non più l'oggetto misurato (cioè il corrispondente codice a barre) del programma riduzionistico; ritorna l'ontologia che sembrava esclusa dal programma scientifico.

In base a queste considerazioni si può fare la seguente critica (piuttosto: obiezione) alla Critica della ragion pura di Kant: i trascendentali di Kant sono programmi $P(d \mid h)$ che scelgono l' $h^{*}$ per ogni input $d$; precedono il confronto $h^{*}-d$, invece di esserne la conseguenza.

La figura 26 mostra come si passa da una teoria ingenua - che procede applicando lo stesso algoritmo a situazioni semanticamente più ricche e pertanto implicando una complessità algoritmica $C$ (lunghezza del programma) e una entropia di Kolmogorov $K$ (perdita di informazione per caos deterministico) crescenti - ad una teoria efficace che, utilizzando elaborazioni linguistiche, porti a bassi $\mathrm{C}$ e $\mathrm{K}$.

\section{DOMANDA 8 - IN CHE COSA CONSISTE IL SENSO COMUNE?}

\section{Risposta 8 - Il senso comune di Antonio Livi}

Lapproccio linguistico all'indagine scientifica, delineato in questa Parte A, offre una base concettuale per accettare i punti di vista del filosofo Antonio Livi, punti di vista che qui di seguito presentiamo. 
Senso comune è il termine utilizzato da Livi per individuare le certezze naturali e incontrovertibili possedute da ogni uomo. Si tratta non di una facoltà o di strutture cognitive a priori ma di un sistema organico di certezze universali e necessarie che derivano dall'esperienza immediata e sono la condizione di possibilità di ogni ulteriore certezza. Livi ha precisato quali siano queste certezze e ha provato che esse sono in effetti il fondamento della conoscenza umana. Il senso comune comprende:

1. l'evidenza dell'esistenza del mondo come insieme di enti in movimento;

2. l'evidenza dell'io, come soggetto che si coglie nell'atto di conoscere il mondo;

3. l'evidenza di altri come propri simili;

4. l'evidenza di una legge morale che regola i rapporti di libertà e responsabilità tra i soggetti;

5. l'evidenza di Dio come fondamento razionale della realtà, prima causa e ultimo fine, conosciuto nella sua esistenza indubitabile grazie a una inferenza immediata e spontanea, la quale lascia però inattingibile il mistero della sua essenza, che è la Trascendenza in senso proprio. Queste certezze sono a fondamento di un sistema di logica aletica su base olistica. 



\section{PARTE B}

\section{LA FISICA}

\section{Il programma della fisica}

Per evitare la notazione specialistica delle formule matematiche, si presenteranno i fatti della fisica in maniera colloquiale; ad esempio,

\section{lavoro $=$ forza $\mathrm{x}$ spostamento}

Con questo approccio discorsivo, il lettore può familiarizzarsi con fatti della cultura scientifica - quali ad esempio l'interferenza delle onde, o lo spettro del Sole -, e quindi acquisire una informazione in modo sufficientemente critico da non ingenerare equivoci sul ruolo che la scienza può avere come strumento interpretativo del reale.

Nel 1991 ho scritto con mia moglie il libro I simboli e la realtà (Jaca Book; d'ora in poi citato come $S R$ ), sui poteri e limiti dei nostri simboli concettuali nel rappresentare la realtà. Ho qui ripreso largamente la parte centrale di $S R$.

Per chi ritenesse sufficiente fermarsi all'euristica, devo far presente che l'ordine in cui si consolidano le nostre visioni del mondo è spesso invertito: prima cioè formuliamo il modello matematico di un fenomeno, e solo dopo - una volta che, giocando con varianti dei parametri a disposizione, abbiamo esplorato teoricamente diverse situazioni possibili e verificato se nella realtà si realizzano - siamo in grado di distillare una spiegazione euristica. Il tentare con parametri diversi corrisponde, nell'indagine scientifica, a quanto facciamo per catturare gli aspetti di un oggetto nuovo: gli giriamo attorno per apprezzarne punti di vista diversi. In effetti ogni nostra percezione visiva è il risultato di una proiezione 2D (bidimensionale) sulla retina. Solo il confronto tra le varie percezioni $2 \mathrm{D}$ memorizzate ci permette di ricostruire l'oggetto in $3 \mathrm{D}$, cioè nello spazio tridimensionale in cui sia noi sia l'oggetto siamo immersi.

La sequenza dei processi scientifici creativi dunque non è prima la comprensione euristica e poi, con funzione decorativa, il formalismo, ma semmai il contrario: prima ci assicuriamo che le nostre protesi mentali (gli strumenti matematici) ci permettano di controllare una situazione, e solo dopo cerchiamo il perché. Demandare ad altri la parte formale ed accontentarsi dell'euristica vuol dire non partecipare al processo creativo ma guardare come altri lo fanno, da spettatori, come se fosse una divulgazione televisiva. 
La fisica (dal greco physis, tradotto in latino con natura) indica la scienza delle cose osservate.

La fisica moderna nasce col programma di Galileo, enunciato nella lettera a M. Welser del 1610: «non tentare le essenze [cioè non cercare di descrivere le nature delle cose, con le conseguenti ambiguità legate a punti di vista soggettivi]ma contentarsi delle affezioni quantitative». Il programma è stato esemplificato con la mela della figura 19 nella Parte A.

Ogni quantità misurata (sapore, colore...) è codificata da un numero; una collezione di numeri (come le barre del codice dei prezzi al mercato) individua un oggetto. Discuteremo in seguito se questa frammentazione sia esauriente, cioè assicuri una descrizione completa dell'oggetto. Dopo Newton, si tenta di descrivere l'oggetto solo con posizioni e velocità delle particelle costituenti.

Le correlazioni osservate fra quantità diverse suggeriscono relazioni matematiche fra i numeri corrispondenti (induzione) o fra il valore $n(t) \mathrm{di}$ una quantità a un certo tempo $t$ e il valore $n\left(t^{\prime}\right)$ a un tempo successivo $t^{\prime}$ (predizione).Il modello è l'insieme delle quantità misurate e codificate in numeri $n, m, \ldots$ (concetti) più le relazioni matematiche (leggi) che collegano questi numeri, e le cui soluzioni anticipano lo stato futuro del pezzo di mondo che stiamo investigando.

Si pone allora la domanda: la fisica è modello o metafora del mondo?

Modello è una collezione di termini univoci, dal cui gioco mutuo emergono predizioni affidabili. Invece metafora è un trasferimento di significato da un ambito cognitivo a un altro, e pertanto adattabile a circostanze diverse.

Prendiamo ad esempio l'apprendimento di una lingua; può realizzarsi:

- per modello: con grammatica e vocabolario;

- per metafora: direttamente nel vissuto, come fanno i bambini.

La fisica non può ridursi a modello; è questo il cuore della complessità.

Intanto elenchiamo le modalità di interrogazione del mondo, per estrarne affezioni quantitative. Sono veicoli di informazione per percezioni sensoriali:

- molecole (olfatto, gusto);

- contatto (tatto);

- onde (udito, vista).

Nel caso di apparati di misura, possiamo in più usare fasci di particelle (elettroni, neutroni ecc.).

Limitiamoci alla vista: non possiamo risolvere oggetti di taglia inferiore alle lunghezze d'onda a cui il nostro occhio è sensibile. Dobbiamo perciò distinguere fra visione diretta (anche se mediata da strumenti che magnificano l'oggetto, quali i microscopi e telescopi) e visione indiretta. Nella visione diretta ad esempio della mela, catturiamo una immagine bi-dimensionale (2D) sulla retina dell'occhio o sulla superficie sensibile della macchina fotografica; confrontando diverse immagini da prospettive diverse, ci co- 
struiamo l'aspetto tri-dimensionale (3D): in effetti, 'girando' attorno alla mela, le varie immagini sono compatibili e si raccordano fra loro (Fig. 31).

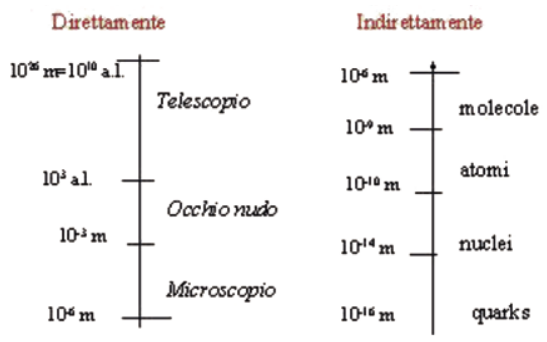

Figura 31 - Come vediamo: l'Universo osservabile, direttamente o indirettamente.

Invece, nella visione indiretta dobbiamo bombardare l'oggetto non visibile con proiettili sonda e osservare il ventaglio di direzioni su cui questi proiettili sono sparpagliati dopo avere interagito con l'oggetto. La stessa sonda può dare luogo a risposte diverse; quindi la singola risposta non è significativa, ma bisogna accumularne una gran quantità e associare l'informazione alla distribuzione dell' insieme delle risposte. E qui nasce una limitazione di principio a qualunque nostra osservazione. Semplifichiamo l'oggetto ignoto come una pallina senza struttura interna; vogliamo allora saperne posizione e velocità per utilizzarle in una ricostruzione newtoniana del mondo.

Ma per un'accurata localizzazione della posizione, dobbiamo usare proiettili energetici, che disturbano la velocità; e, viceversa, per misurare la velocità perdiamo di accuratezza nella posizione. Si ha cioè la relazione di indeterminazione di Heisenberg: il prodotto delle incertezze in posizione e velocità non può scendere sotto un valore minimo. Se costruiamo un piano posizione-velocità e cerchiamo di individuare una coppia di valori $(x, v)$, non possiamo localizzare il punto corrispondente ma abbiamo una curva di probabilità a campana che si appoggia sull'area di Heisenberg. Se cerchiamo di migliorare la risoluzione in una direzione, la peggioriamo nell'altra (Fig. 32).

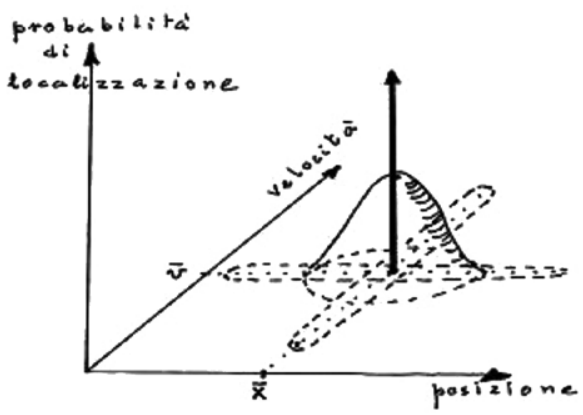

Figura 32 - Incertezza posizione-velocità nelle misure indirette. 
Il limite vale per qualunque misura; solo che l'area di Heisenberg è così piccola che nelle misure dirette non ce ne accorgiamo e reputiamo di aver misurato la coppia senza che una misura disturbi l'altra.

Conclusione: il lessico della fisica è accurato solo a 'grana grossa'; se scendiamo a grana fina, ci sono sovrapposizioni fra le campane di incertezza legate a misure distinte (che dovrebbero codificare concetti diversificati, cioè termini distinti del lessico della fisica). Il formalismo in tal caso non è più sufficiente: occorre introdurre anche una informazione con-testuale, così come avviene nel linguaggio ordinario.

Nel discorso scientifico rimane una componente metaforica ineliminabile, che peraltro è il segreto della creatività, in quanto permette di esplorare scenari non inclusi nella formulazione di partenza. È per questo che il programma della fisica appare come aperto e non ha senso porsi la domanda: dove finisce l'indagine fisica?

\section{Complessità formale, algoritmica e semantica}

La complessità nasce dal tentativo di una costruzione logica del mondo a partire dalle «affezioni quantitative» in cui frantumiamo un oggetto di esperienza, piuttosto che «tentarne l'essenza» (Galileo, 1610). Il tentativo è motivato dalla convinzione che si possa raggiungere una descrizione completa di un oggetto di esperienza attraverso i suoi atomi di significato (esempio della mela: odore, colore, sapore, forma, oppure, più radicalmente, gli atomi di cui è composta). Ogni oggetto diventa così un elemento definito di un insieme di Cantor (Carnap, 1931).

Tre limiti a questo programma riduzionistico corrispondono a tre possibili caratterizzazioni della complessità: formale, algoritmica e semantica.

A) Formale

1931, Kurt Goedel, teorema di indecidibilità: estraendo tutte le possibili conseguenze da un corpodi assiomi, prima o poi si arriva a una proposizione indecidibile all'interno della teoria (cioè elaborando gli assiomi con un algoritmo unico, quale la logica formale: v. Fig. 9); l'equivalente 'macchinistico' fu enunciato da Alan Turing 1936,come impossibilità di una macchina universale di calcolo (cioè un computer) di fermarsi completando l'esecuzione per un generico input.

Dunque, non possiamo costruire una teoria chiusa del mondo, cioè dedurre tutti gli aspetti della nostra esperienza a partire da un numero finito di assiomi.

в) ALGoritmicA( 1970, Kolmogorov e Chaitin)

La complessità è il costo di un calcolo; essa ha pertanto due aspetti:

- spaziale: quantità di risorse di macchina da impegnare;

- temporale: tempo necessario all'esecuzione. 
Esploriamo questo secondo aspetto. Sia $t_{1}$ il tempo necessario per descrivere un singolo elemento e si abbiano $N$ elementi. Se le loro proprietà si sommano (come i fagioli in un sacco), cioè se l'oggetto composto di $\mathrm{N}$ elementi è cresciuto per giustapposizione, allora il tempo totale necessario $T$ è:

$$
T=N t_{1}
$$

Se invece la crescita è per intussuscezione (uso un vecchio termine della filosofia della natura che vuol dire che gli elementi non si limitano a giustapporsi, ma si accoppiano fra di loro con modalità non prevedibili a partire da una ispezione del singolo elemento; esempio: la collezione di vocaboli di un lessico, disposti convenzionalmente in ordine alfabetico nel dizionario, e come invece si organizzano nel verso di un poeta) allora il tempo totale non è la semplice somma dei tempi individuali, e addirittura l'estensione ("size") $N$ del sistema composto può figurare ad esponente di una certa base, ad esempio 10:

$$
T^{\prime}=\left(t_{1}\right) 10^{N}
$$

Per esempio, sia $t_{1}=1$ microsecondo $=10^{-6} \mathrm{sec}, N=10$, allora:

$$
T=10 t_{1}=10^{-5} \mathrm{sec} ; \text { invece } T^{\prime}=t_{1} 10^{10}=10^{4} \mathrm{sec}
$$

che è ancora praticabile (si tratta di meno di tre ore); ma se $N=100$, allora:

$$
T=100 t_{1}=10^{-4} \mathrm{sec} \text {, invece } T^{\prime}=t_{1} 10^{100}=10^{94} \mathrm{sec}
$$

molto più lungo dell'età dell'Universo (che è circa $10^{7} \mathrm{sec} / \mathrm{anno} \times 10^{10} \mathrm{an}$ $\mathrm{ni}=10^{17} \mathrm{sec}$ ).

c) Semantica

La complessità semantica è la più fondamentale: rappresenta il limite della presunzione ideologica della fisica dei componenti elementari di essere l'unica descrizione rilevante del reale, un esempio paradigmatico di riduzionismo. È stata già discussa con riferimento alla figura 8.

Supponiamo di scomporre una mela nelle sue affezioni quantitative, cioè nelle proprietà distinte che possiamo misurare: sapore, odore, colore, forma ecc. Ognuno di questi punti di vista corrisponde ad un apparato di misura che fornisce un numero. Se abbiamo scelto un numero adeguato di indicatori, la collezione ordinata dei numeri corrispondenti a ciascuna misura dovrebbe caratterizzare una mela. Ciò può bastare per il rifornimento del magazzino di un mercato, però non esaurisce la realtà della mela. In effetti l'intero (la mela) precede le misure separate, che forniscono quantità derivate, non primitive: si appoggiano alla mela ma una volta enucleate (tecnicamente: astratte) non permettono la ricostruzione logica della mela. Non siamo mai sicuri che nel futuro non compaia un 
ulteriore punto di vista inedito che sia misurabile e che arricchisca la descrizione della mela.

Gli esempi più convincenti vengono dalla linguistica. Sappiamo bene che prima nasce il linguaggio articolato, la poesia, le favole, e poi da queste si estrae un lessico. L'operazione inversa, partire dal vocabolario e programmare al computer un testo letterario, è fallimentare. In questo ordine di complessità rientra la traduzione da una lingua all'altra. Esistono macchinette per cavarsela alla stazione o al ristorante in Cina; ma per un testo elaborato, per esempio Omero, il traduttore non è automatico: piuttosto è un bilingue che vive le situazioni di Ulisse in greco e le racconta in italiano. Questo ruolo del traduttore è discusso in un bel libro, Il grande codice (la Bibbia e la letteratura) di Northrop Frye, in cui l'autore si pone il problema: come mai la Bibbia scritta in ebraico (lessico di 5.000 vocaboli) può essere tradotta in inglese (il cui lessico comprende 500.000 vocaboli)? La risposta è che una procedura complessa come la traduzione letteraria non può essere traslata in un elenco di operazioni di macchina, e perciò non può essere automatizzata.

\section{Caos deterministico: esempi di applicazione a processi percettivi e ad orologi biologici}

Il caos deterministico è legato alla mancanza di stabilità trasversale delle traiettorie (come visto in Fig. 23). Rappresentiamo una traiettoria, di quelle che abbiamo imparato a calcolare con le regole nate con Newton e sviluppate nei secoli successivi, con un tratto di retta. In direzione ortogonale alla traiettoria possiamo avere un paesaggio dell'energia che sembra una valle o un colle. È un modo figurato per rispondere alla domanda: quanto costa in energia (che rappresentiamo in questo schema come altezze) turbare la traiettoria a partire da un certo punto? Nel paesaggio a valle, un eventuale errore nella condizione iniziale viene riparato al passare del tempo e ricade sulla retta; nel paesaggio a colle, viene amplificato e rende incerta la previsione sul resto della traiettoria. Da qui l'apparente ossimoro: caos perché non si può prevedere il futuro al di là di un tempo limitato (corrispondente alla caduta dal colle), deterministico perché non è l'effetto statistico di una folla di perturbazioni incontrollate, ma insorge in sistemi semplici, descritti da un numero piccolo di equazioni dinamiche.

Una traiettoria dinamica (schematizzata in Fig. 23 dalla linea spessa con freccia) per un sistema dinamico descritto da due equazioni - diremo: con due gradi di libertà (2D) - è stabile trasversalmente, nel senso che, se esploriamo punti vicini alla traiettoria, i moti che partono da questi tendono a convergere verso la traiettoria originale; rappresentiamo ciò come il confinamento dei punti sulla linea di fondo-valle. Poincaré (1890) (vedi Arecchi, 2007) mostrò che basta passare da 2 a 3 equazioni (diremo: da 2 a 3 dimensioni dinamiche, abbrevieremo con 2D e 3D) per- 
ché possa insorgere il passaggio da valle a colle. Da tre gradi di libertà (3D) in su, è dunque possibile una instabilità trasversale, in cui la traiettoria è arroccata sulla cima di un colle; qualunque incertezza ad un certo istante si trova amplificata nei tempi successivi dalla caduta a destra o a sinistra del colle: questa incertezza che cresce nel tempo è detta caos deterministico. Vedremo che è essenziale avere forze non lineari (il termine gergale sarà chiarito nella Parte $\mathrm{C}$ ) altrimenti qualunque sistema, per ricco che sia di componenti, può dinamicamente scomporsi in sistemi regolari giustapposti.

Descriviamo una applicazione del caos al sistema solare. Finché consideriamo il problema a 2 corpi Terra-Sole siamo nel caso stabile; ma se teniamo conto di altri corpi, la dinamica di un qualunque pianeta è instabile, con una fuga dall'orbita ideale su tempi dell'ordine di 500.000 anni: è perciò che non ce ne siamo accorti nei 3.000 anni di osservazioni astronomiche! Una possibile conseguenza è che 65 milioni di anni fa un asteroide, che fino ad allora aveva orbitato fra Marte e Giove, intraprendendo un'orbita allungata, sia caduto sulla Terra causando una catastrofe ecologica che ha determinato l'estinzione di massa dei dinosauri.

Il caos è oggi applicato a molti fenomeni e ne spiega l'insorgenza di apparenti anomalie, quali un tornado in meteorologia, o una crisi nei mercati finanziari, o un black-out nella distribuzione di energia elettrica in una regione.

Una volta scoperto il caos nel singolo sistema dinamico, cosa succede se ne mettiamo insieme un numero grande, con accoppiamenti mutui, con reti di feedback (chiamiamo feedback il fatto che un sito A, ricevendo un segnale da $B$, reagisca mandando indietro un segnale risposta che a sua volta modifica B)? La grossa sfida è il controllo del caos (vedi Fig. 24), che permette di trasformare un oggetto imprevedibile in uno con comportamento regolare.

Consideriamo il funzionamento di un neurone. Un neurone (cellula cerebrale) è un sistema dinamico non lineare e pertanto genericamente affetto da caos; esso si accoppia in rete agli altri di una certa zona cerebrale, ad esempio la corteccia visiva o auditiva, contribuendo ad una percezione definita.

Presentiamo il codice temporale che sta alla base del feature binding (collegamento fra le attività di neuroni esposti a stimoli localmente diversi, ma che devono contribuire a formare una percezione significativa) (Fig. 33). Per collegarsi con gli altri, un neurone codifica l'informazione che a sua volta ha ricevuto in un potenziale d'azione che consiste di brevi impulsi di durata $1 \mathrm{msec}$ (spikes) separati in media di $25 \mathrm{msec}$. Gli intervalli temporali fra spikes successive (ISI = Inter Spike Interval) dipendono dallo stimolo, cioè codificano uno specifico segnale: un po'come il codice a barre di un prodotto.

Come si può vedere in figura 33, tutti quei neuroni nei cui campi recettivi entrino parti del gatto (i cerchietti neri della figura) emettono treni di spikes sincronizzati. Lo stesso accade per la donna (i cerchietti bianchi). 

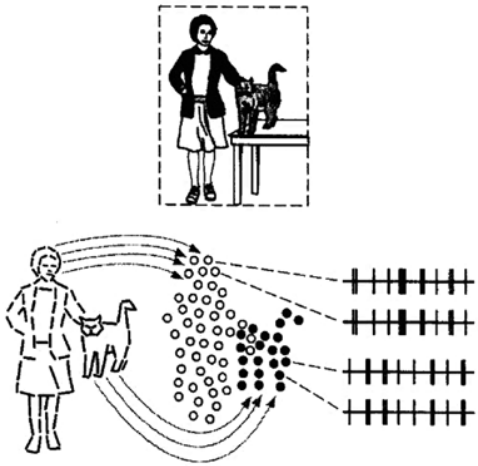

Figura 33 -Feature binding: la donna e il gatto sono rappresentati ognuno da un insieme di neuroni, indicati rispettivamente dai cerchi vuoti e da quelli pieni. Questi insiemi racchiudono i neuroni che rivelano i dettagli specifici degli oggetti visuali (come, ad esempio, lorientazione dei segmenti di contorno). Le relazioni tra i dettagli possono essere codificate grazie alle correlazioni temporali: neuroni che fanno parte dello stesso insieme scaricano impulsi elettrici in sincronia, mentre non ci sono relazioni temporali tra neuroni che si riferiscono ad oggetti diversi.

Si noti che così stiamo introducendo un codice temporale (CT) ben più sofisticato della semplice posizione dell'ago (PA) di uno strumento di misura (oppure display digitale). CT permette una maggiore ricchezza di informazione. Supponiamo ad esempio che la minima distanza fra due spikes successive sia $3 \mathrm{msec}$ e che il tempo di lettura per avere una percezione sia $180 \mathrm{msec}$ (sono dati abbastanza vicini a quelli fisiologici); avremo pertanto $180 / 3=60$ finestre temporali $0 / 1$ (c'è/non c'è impulso) sull'intervallo totale di misura. Questo permette di costruire $2^{60}$, cioè circa $10^{20}$, possibilità diverse (messaggi), che differiscono uno dall'altra per le posizioni e i numeri di 0 e di 1 ; di volta in volta, l'informazione è codificata dalla sequenza binaria, ad esempio $001011100 \ldots$ e così via per 60 volte.

Invece, in PA l'ago dello strumento (o il display digitale) darebbe solo 60 posizioni diverse, cioè 60 possibili messaggi, che differiscono uno dall'altro per la posizione (ogni volta unica!) del cursore.

Prima ancora che fossero comparsi gli animali con cervello, la natura aveva già sfruttato l'esplosione combinatoria per arricchire la panoplia dell'informazione; con solo 92 atomi (anzi con molto meno) ha combinato centinaia di migliaia di molecole.

Tuttavia, come fa un neurone ad adattare l'ISI al variare dello stimolo? Il segreto sembra consistere in una dinamica detta omoclinica $(\mathrm{HC}=\mathrm{Ho}-$ moclinic Chaos), cioè nel continuo ritorno a una sella, come mostrato in figura 34: la traiettoria si avvicina alla sella da una direzione a e ne scappa dalla direzione $\gamma$; in 3D (altrimenti non c'è caos!) il sistema rallenta più 0 meno attorno alla sella, pertanto il periodo del ciclo omoclinico è varia- 
bile. Inoltre, nella zona della sella il sistema è molto sensibile alle influenze esterne (in gergo diciamo suscettibile) per cui il tempo di permanenza può essere alterato per effetto di uno stimolo esterno. Ciò permette a un gruppo di neuroni accoppiati di influenzare mutuamente i propri ISI e di sincronizzarsi, oscillando tutti con lo stesso ritmo.

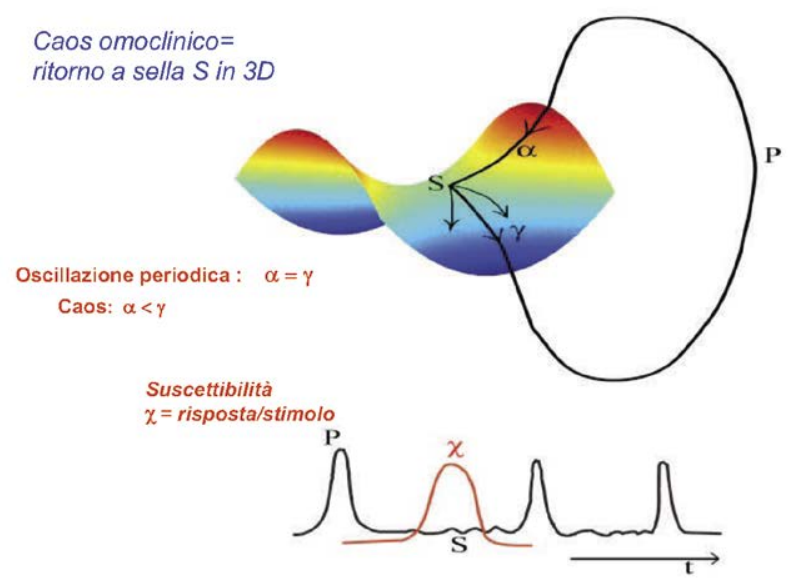

Figura 34 - Un sistema dinamico abbia traiettorie chiuse, con il ritorno ad ogni giro ad un punto di sella $S$, a cui si avvicina più rapidamente di quanto ne scappi $(\alpha<\gamma)$. Se ora rappresentiamo la posizione lungo la traiettoria con un segnale verticale in funzione del tempo, i larghi percorsi P lontano dalla sella daranno impulsi P tutti uguali, separati però da un intervallo più o meno lungo che rappresenta il tempo di permanenza nella zona di sella. Conviene visualizzare il moto lungo lorbita (detta in gergo omoclinica per indicare che ritorna sempre alla stessa sella) come il moto di una pallina pesante: è intuitivo che dopo l'avvicinamento a $S$ e prima della fuga da $S$, la pallina rallenta e diventa perciò suscettibile a variare il suo tempo di permanenza attorno a $\mathrm{S}$ in base ad uno stimolo esterno. Il sistema può perciò essere sincronizzato da un segnale.

I neuroni dei primi stadi corticali rivelano aspetti elementari del loro campo recettivo, quali ad esempio barre orientate; quindi ci aspetteremmo che i vari neuroni di un gruppo abbiano ISI diversi, a seconda dello stimolo che ciascuno riceve. Per combinare gli ISI in una sola sequenza sincronizzata su tutti i neuroni esposti al gatto occorre riaggiustare la soglia di ciascun neurone, in modo che l'emissione della singola spike sia un compromesso fra lo stimolo che arriva dalla retina e la propensione del neurone a sparare la spike, propensione stabilita appunto dalla soglia. Il riaggiustamento avviene con segnali top-down corrispondenti ad aspettazioni della memoria semantica, la quale fa riferimento ad apprendimenti pregressi in cui ha immagazzinato la percezione del gatto. E questa l'ipotesi più plausibile, formulata da $\mathrm{S}$. Grossberg nel 1976 e detta ART (Adaptive Resonance Theory). 
Il meccanismo di sincronizzazione descritto si basa sul ruolo cruciale della sella: quando un sistema dinamico si comporta così diciamo che ha una grande propensione a formare una rete sincronizzata. Tutti gli orologi biologici devono essere adattabili a situazioni ambientali diverse, perciò è plausibile che abbiano un comportamento dinamico simile a quello appena delineato.

In Tabellal presentiamo i più importanti orologi biologici. I periodi indicati per ciascuno sono medi, non vanno ritenuti dei valori fissi come sarebbe per un orologio a quarzo, in modo che possano modificarsi per rispondere ad esigenze diverse. Ad esempio, il pace-maker cardiaco ha un periodo medio di $1 \mathrm{~s}$, ma se durante uno sforzo fisico (una corsa) c'è richiesta di sangue ossigenato allora il periodo può ridursi a $0,5 \mathrm{~s}$ o meno, e durante il riposo notturno può aumentare a $1,5 \mathrm{~s}$ o più.

Tabella 1 - I principali ritmi biologici e i periodi corrispondenti.

\begin{tabular}{ll}
\hline Ritmi neuronali & $0,01-10 \mathrm{~s}$ \\
\hline Ritmo cardiaco & $1 \mathrm{~s}$ \\
\hline Oscillazioni del calcio & $1 \mathrm{~s}$ fino a molti minuti \\
\hline Oscillazioni biochimiche & $1-20$ minuti \\
\hline Ciclo mitotico & 10 min fino a $24 \mathrm{~h}$ \\
\hline Ritmi ormonali & $10 \mathrm{~min}$ fino ad alcune ore \\
\hline Ritmi circadiani & $24 \mathrm{~h}$ \\
\hline
\end{tabular}

Ladattabilità (fitness) è una delle tre caratteristiche essenziali per la vita nella visione di Darwin.

\section{Fisica e fisiologia}

L'interesse del fisico ai processi fisiologici è di vecchia data, da Aristotele a Helmholtz. In effetti, la distinzione tra fisica e fisiologia è recente, quando la fisica - invece di essere la scienza di tutti i fenomeni naturali - si è organizzata attorno ad un programma riduzionistico, limitandosi a studiare quei fenomeni che siano riconducibili a interazioni tra componenti elementari.

La crisi del programma riduzionistico è legata allo studio dei fenomeni complessi, in cui la dinamica non lineare induce comportamenti di sistema non prevedibili a partire dalla descrizione dei componenti.

Il riduzionismo credeva di poter spiegare i comportamenti globali come somma di comportamenti individuali; questa tendenza era appoggiata da successi sperimentali nei campi più disparati, dalla fisica dei processi atomici e subatomici alla descrizione dei processi di rivelazione della luce sulla retina, e così via. Per contro gli approcci olisti- 
$c i$, di tipo psicologia della Gestalt, che insistevano sull'unitarietà della percezione globale, il cui significato non può emergere dalla semplice somma dei contributi particolari, sembravano arbitrarie petizioni di principio in quanto non in grado di stabilire correlazioni logiche con i processi elementari.

Se l'approccio riduzionistico avesse avuto una validità universale, allora combinando elementi di percezione elementari si sarebbe dovuto riuscire a modellare la percezione globale, $\mathrm{o}$ in un computer o come protesi di un robot. I tentativi in questa direzione non hanno avuto molto successo. La ragione è legata alla dinamica non lineare: per una interazione non lineare non vale la regola della somma, ma possono emergere comportamenti globali nuovi, non prevedibili dallo studio dei singoli componenti.

Sulla scia del successo nell'identificare procedure di riconoscimento elementari, associate a singoli neuroni, si pensò di avere risolto il problema della conoscenza. Ma in un mondo siffatto in cui ogni neurone deve codificare una specifica informazione ed essere deputato solo a quella (la teoria del 'neurone della nonna') l'aumento di conoscenza implicherebbe una crescita spropositata del numero di neuroni. Invece, come visto nel paragrafo precedente, la congettura vincente è che i neuroni abbiano un'ulteriore libertà, cioè la possibilità di una codifica temporale. Al di sopra di una soglia, lanciano treni di impulsi nervosi la cui frequenza (numero di impulsi per unità di tempo) è proporzionale alla distanza dalla soglia. Questo suggerisce come spiegare una percezione unitaria di una figura con differenti livelli di luminosità, colore, forma ecc.: basta che i neuroni elementari deputati a rivelare ciascuna di queste caratteristiche sincronizzino le loro frequenze di scarica in modo da costituire un collettivo che in quel momento porta a individuare quella configurazione (si veda il gatto di Fig. 33), lasciando però i vari neuroni liberi di riorganizzarsi successivamente in altri collettivi (feature binding).

Ma chi riaggiusta le soglie? Non certo gli stimoli che vengono dalla retina, cioè dal 'basso' (bottom-up), in quanto questi differiscono da un neurone all'altro a seconda che i rispettivi campi ricettivi siano fonte di più o meno segnale. Occorre che ci sia un ritorno, un feedback, dal bagaglio di memorie pregresse, dove sono registrati riconoscimenti precedenti di figure significative. Ciò può avvenire con un ri-aggiustamento delle soglie dovuto a segnali da aree corticali superiori, cioè dall"alto' (top-down). Secondo questa congettura le categorie già memorizzate suggeriscono un'ipotesi interpretativa (aspettativa). Se combinando le due correnti di stimoli (top-down e bottom-up) si finisce con il sincronizzare stabilmente un collettivo di neuroni, che così fornisce un'immagine significativa, allora si crea una risonanza, cioè i valori di stimolo vengono confermati e si arriva a una percezione coerente. Questo è il succo della ART.

Feature binding e ART sembrano non appartenere all'universo del fisico, il quale un tempo si limitava ai processi elementari. Invece la fisica della complessità offre un quadro entro cui collocare convenientemente anche queste congetture, a patto che quella parola misteriosa (perlome- 
no: per la fisica) 'significativo', evidenziata due volte nella frase precedente, acquisti un senso.

\section{Dinamica ed energia}

I greci avevano sviluppato la geometria (specie con Euclide) e la statica, cioè i legami di equilibrio fra corpi fermi (con Archimede), ma non avevano capito come un corpo si muove. Ciò era fra l'altro dovuto alla mancanza di buoni orologi. L'osservazione di Galileo (1581) dell'isocronismo delle oscillazioni del pendolo fornì un orologio affidabile, e cinquant'anni più tardi, nei Discorsi (1638) Galileo creava la dinamica come scienza del moto.

Compito specifico della fisica, da Galileo e Newton in poi, è stato - come si è detto - quello di isolare, dalle osservazioni, pochi elementi quantitativi traducibili in numeri. Rispetto alla ricchezza del reale, la scelta non poteva non essere difficile. Per questioni di raccordo culturale, Galileo, Newton e i loro contemporanei selezionarono fra i molti concetti correnti nei dibattiti scolastici sulla fisica quelli che apparivano più sensati $\mathrm{e}$ tentarono di associare a ciascuno una specifica operazione di misura. Ad esempio, l'idea intuitiva di 'forza' come causa del moto porta subito ad enunciare due risultati.

a) Come si indica che si è creato un moto? Gli aristotelici dicevano: attraverso la velocità. Galileo obiettò che la velocità si conserva anche in assenza di forze; è pertanto la variazione di velocità, o accelerazione, che va presa come proporzionale alle forze.

b) Se ci sono due forze opposte, queste si possono compensare (equilibrio) in modo tale che non ci sia moto. Allora, per misurare una forza, basta misurare una forza compensatrice più accessibile alla misura.

Ricordiamo due termini, entrati nell'uso corrente:

- cinematica $=$ studio puramente geometrico del moto, indipendentemente dalle cause che lo generano;

- dinamica = studio del moto correlato alle cause (le forze).

La fisica del Seicento e del Settecento è stata prevalentemente dinamica. Anzi, il successo dei primi modelli di Galileo e Newton generò la credenza che tutta la fisica si potesse ridurre al moto reciproco delle particelle che compongono un corpo per effetto delle forze mutue. Questa credenza (meccanicismo) ebbe un supporto filosofico nel parallelo tentativo di riduzione delle 'sostanze' (in senso aristotelico) ad un'unica sostanza materiale: la res extensa (introdotta da Cartesio, il quale aveva sviluppato un dualismo opponendo alla res extensa una res cogitans propria solo dell'uomo).

E bene chiarire perché si è parlato dei 'modelli' di Galileo (piano inclinato, pendolo) e Newton (moto Terra-Sole): il modello rappresenta un possibile modo di operare della realtà. 'Forse' è il modo vero, ma di ciò saremmo sicuri solo se la nostra traduzione della realtà nello spa- 
zio dei simboli fosse univoca. Questa era la profonda convinzione di Galileo e Newton, ma la fisica moderna ha esplorato modi alternativi di tradurre in simboli lo stesso pezzo di mondo, e questi modi alternativi dimostrano spesso una fecondità notevole. Diremo allora che per una stessa realtà esistono più modelli plausibili, e che la scelta dipende dalla fecondità.

Ma torniamo alla dinamica, e consideriamo un moto molto semplice: quello di una pallina che cade su un piano inclinato (Fig. 35). Rappresentiamo la forza con vettori, cioè con segmenti orientati secondo la direzione delle forze e di lunghezza proporzionale alle forze stesse.
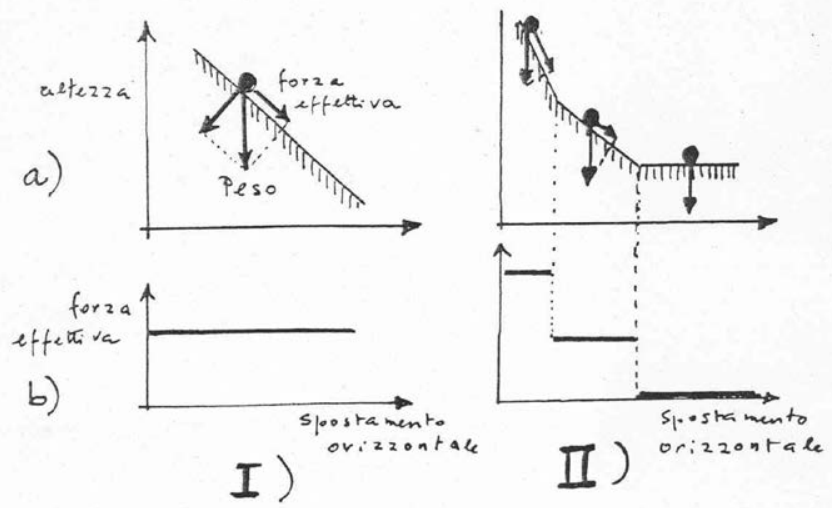

Figura 35 - a) Pallina pesante che cade su uno (I) o tre (II) piani inclinati privi di attrito. b) Forze corrispondenti.

Nel caso della forza peso, avremo un vettore verticale che ha lo stesso valore in qualunque posizione, perché il peso non dipende dall'altezza. Ora, sappiamo fin da Archimede che se due forze tirano in direzione diversa, la forza risultante si ottiene sommando con la regola del parallelogramma (cioè è la diagonale del parallelogramma che ha per lati le due forze componenti). Facciamo l'operazione opposta: decomponiamo il peso, verticale, in una componente perpendicolare al piano (che non avrà nessun effetto, perché il piano è rigido, cioè non deformabile) e una parallela, che sarà l'unica parte effettiva. Questa parte varia con l'inclinazione, come mostrato nella parte II della figura.

Invece, nel caso in cui la pallina si muova sul fondo di una tazza, la forza cambierà da punto a punto (Fig. 36). Disegniamo due assi graduati, uno orizzontale su cui misuriamo gli spostamenti rispetto al fondo tazza (+ a destra, - a sinistra) e uno verticale su cui misuriamo le altezze della pallina. Se la forma della tazza è parabolica, si dimostra facilmente che la forza varia in modo proporzionale allo spostamento, pertanto il suo grafico è una retta. 


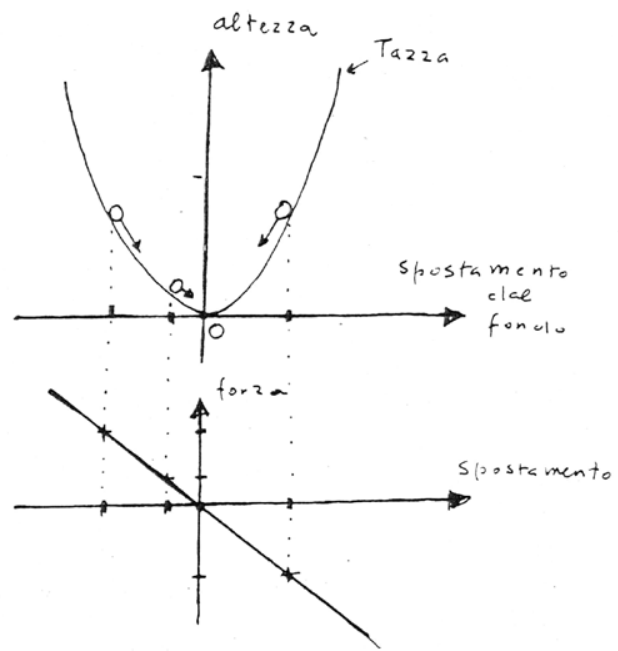

Figura36 - a) Pallina pesante che si muove senza attrito in una tazza di forma parabolica. A diverse posizioni corrispondono forze con diverse direzioni (+ se verso destra, - se verso sinistra) e intensità (la lunghezza della freccia denota l'intensità). b) Diagramma delle forze al variare dello spostamento del fondo della tazza.

Quando un moto è indotto da una forza che è proporzionale allo spostamento, diremo che la dinamica è lineare. Per le dinamiche lineari vale la sovrapposizione degli effetti: possiamo cioè valutare tutte le proprietà dinamiche per una forza unitaria e, se la forza aumenta o diminuisce di intensità, limitarci ad alterare le proprietà di fattori di scala convenienti senza dover rifare lo studio per ogni valore di forza.

Torniamo alla figura 36. Il fondo tazza è il punto di equilibrio dove la forza si annulla. Se consideriamo anche l'attrito, dopo un po' il moto si spegne e la pallina rimane sul fondo. Se invece idealizziamo un mondo senza attriti, allora il moto continua per sempre. Ma fra dove e dove? Appoggiamo inizialmente la pallina sul lato sinistro della figura. Essa cade e raggiunge il fondo. Qui ha sufficiente velocità per risalire fino a una posizione simmetrica a quella da cui era partita. Qui si ferma e torna indietro, eseguendo oscillazioni per sempre. Nella realtà, l'attrito smorza le oscillazioni, cosicché la pallina risale sempre meno fino a fermarsi sul fondo.

Introduciamo ora un altro concetto: il lavoro. Nel cadere verso il fondo, la pallina 'può' fare lavoro: infatti se la facessimo cadere nelle pale di un mulinello, questo si metterebbe a girare e potrebbe fare qualcosa di utile (macinare farina o dare elettricità mediante una dinamo del tipo di quelle delle bici). Misuriamo il lavoro come prodotto della forza per l'altezza di caduta, o spostamento della pallina:

$$
\text { lavoro }=\text { forza } \mathrm{x} \text { spostamento }
$$


Dove per spostamento si intende la componente nella direzione della forza.

Ad esempio, se devo spostare un libro in uno scaffale più alto in libreria, devo fare un lavoro:

$$
\text { lavoro }=\text { peso } \mathrm{x} \text { altezza }
$$

Viceversa se il libro cade, dallo scaffale alto, il lavoro conseguente alla caduta si trasforma in danneggiamento del libro quando questo arriva sul pavimento. Se un libro va spostato sullo stesso scaffale, in orizzontale, il lavoro è nullo: si pensi al caso ideale di un libro che possa scivolare senza attrito sul piano dello scaffale.

Definiamo energia la potenzialità di lavoro, cioè il lavoro in potenza. Se la forza non è costante, come andranno le cose? Ebbene - e qui vi è l'enorme vantaggio dell'usare il concetto di energia! - l'energia non dipende dalla forma del declivio ma solo dallo stato iniziale e finale. Data qualunque ciotola, la forma della ciotola dà il profilo dell'energia: infatti in qualunque posizione, basta valutare quanto si dista dal fondo per sapere quanto lavoro si può fare.

Anche alla velocità possiamo associare energia. Infatti possiamo fare lo stesso danno al libro lanciandolo orizzontalmente (senza perdita di altezza) contro il muro: chiameremo questa energia cinetica. C'è però una differenza: mentre l'energia di caduta cambia segno in risalita (in discesa la pallina fa lavoro, in risalita deve ricevere lavoro) l'energia cinetica è sempre positiva quale che sia il segno della velocità (verso destra + o verso sinistra -). Ciò è possibile se questa energia è proporzionale al quadrato della velocità: infatti $(+2)^{2}=(-2)^{2}=+4$. Inoltre è proporzionale alla massa: a pari velocità, un camion fa più danno di una bicicletta sbattendo contro un ostacolo.

Se chiamiamo energia potenziale quella che si attualizza nella caduta, possiamo allora leggere il moto della pallina nella ciotola come segue (Fig. 37).

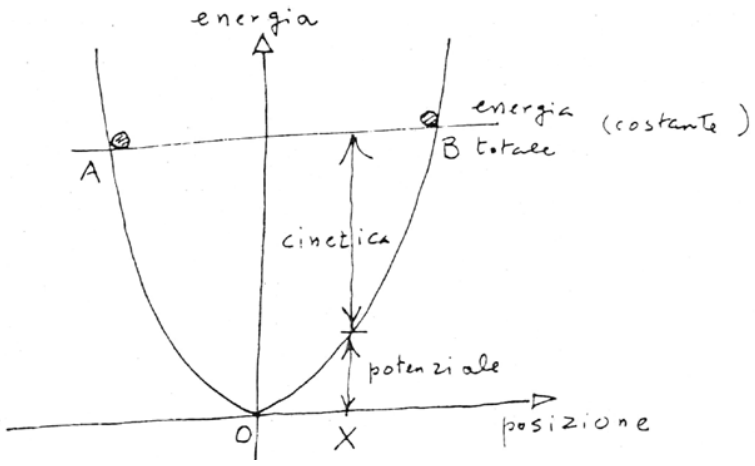

Figura 37 - Suddivisione dell'energia totale tra cinetica e potenziale per ogni posizione $\mathrm{x}$. In posizione 0 l'energia è tutta cinetica. In A e B è tutta potenziale, perciò ivi la velocità è nulla e la pallina torna indietro. 
In A e B l'energia è tutta potenziale. Quando si cade da A ad O, l'energia si trasforma tutta in energia cinetica e l'energia potenziale si annulla. In una posizione generica $\mathrm{X}$ avremo parte cinetica e parte potenziale. $\mathrm{Ri}$ salendo a B la cinetica si trasforma tutta in potenziale e la pallina si ferma. Essa non può superare $\mathrm{B}$ perché l'energia totale non si può creare. Dunque avremo il seguente principio di conservazione dell'energia:

$$
\text { energia totale }=\text { en. potenziale }+ \text { en. } \text { cinetica }=\text { costante }
$$

Se ora capovolgiamo la ciotola e depositiamo la pallina sulla cima (in O), sia che cada verso sinistra (S) sia verso destra (D), la pallina guadagna energia cinetica allontanandosi da $\mathrm{O}$ (situazione di equilibrio instabile). Il corrispondente diagramma di forza è una retta di inclinazione invertita rispetto alla figura 37.

Questa situazione instabile non può andare avanti per sempre. A un certo punto occorre interrompere la curva di potenziale e quindi azzerare in corrispondenza la forza. Ma allora la forza non è più una retta continua.

Quando la forza è rigorosamente rettilinea, la dinamica si dice lineare, quando non lo è dappertutto, la dinamica si dice non lineare.

In seguito descriveremo qualunque dinamica mediante l'energia, cioè diremo di poter fare previsioni sul futuro di un sistema (simbolizzato come una pallina) quando lo avremo immesso in un opportuno paesaggio dell'energia, dove i punti di equilibrio sono tutti i punti S (stabili) e I (instabili), in cui si ha un minimo o un massimo, cioè in cui si annulla la forza; invece punti come X o Y non sono di equilibrio perché si cade (nella direzione della freccia) (Fig. 38).

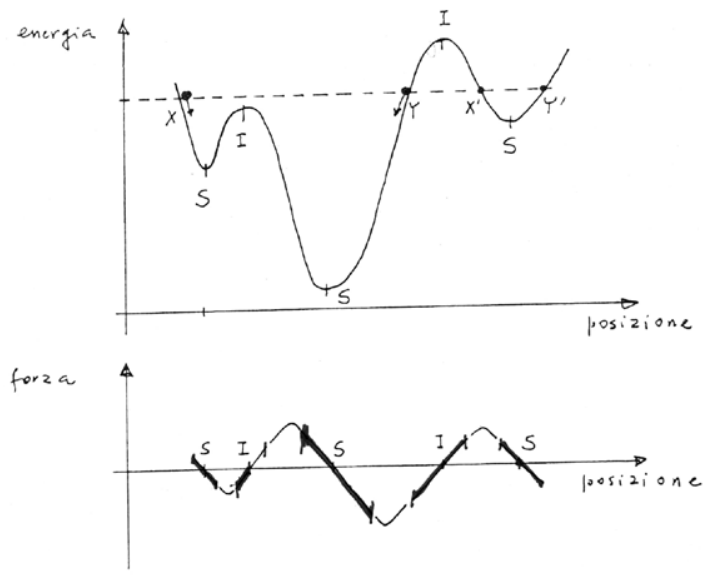

Figura 38 - Paesaggio dell'energia con cinque punti di equilibrio: tre minimi S e due massimi I. La pallina rimane confinata in uno dei due bacini XY o X'Y' dove è stata collocata al tempo iniziale. La forza è approssimabile con un tratto rettilineo solo attorno ai punti di equilibrio. 
Si noti che localmente, cioè attorno ad ogni punto di equilibrio, la dinamica è lineare, ma globalmente la legge di forza non è rettilinea. Supponiamo che l'energia totale di cui dotiamo il nostro sistema corrisponda alla linea orizzontale tratteggiata. Se al tempo iniziale il sistema ha una posizione fra $\mathrm{X}$ e $\mathrm{Y}$, oscillerà per sempre fra $\mathrm{X}$ e $\mathrm{Y}$ scavalcando l'ostacolo intermedio perché, anche quando arriva ad I, ha ancora un residuo di energia cinetica. Se invece è posta fra X' e Y', rimarrà confinata in quel bacino. In ambo i casi, sia che arrivi a $Y$ da destra o a $X$ ' da sinistra, deve invertire il moto perché non ha energia sufficiente per superare la barriera.

Introduciamo delle opportune unità di misura per i processi microscopici. Per sollevare un libro dalla tavola ad uno scaffale, dobbiamo fare lavoro contro la forza di gravità; analogamente per 'sollevare' la carica $e$ di un elettrone della differenza di potenziale elettrico di 1 volt, occorre fornire un lavoro che è dato dal prodotto della carica per 1 volt. Consideriamo questa come l'unità di energia negli scambi microscopici e la abbreviamo con eV (elettrone-volt). Se per esempio un elettrone cade di 10 volt o 10 elettroni cadono di 1 volt, l'energia scambiata è $10 \mathrm{eV}$ (Fig. 39).

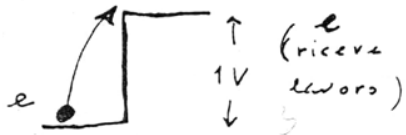

a) carica di barriera

(l'elettrone riceve 1 Volt)

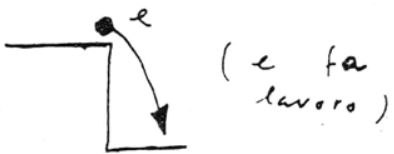

b) scarica

(l'elettrone cade di 1 Volt)

Figura 39 - Processo di carica e scarica di una pila da 1 volt. Nella carica ogni elettrone viene 'promosso' a un livello più alto. Nella scarica, l'elettrone ricade cedendo l'energia accumulata.

L'energia descritta per la pallina, il libro e l'elettrone è dovuta a cambiamento di posizione e può essere restituita con la ricaduta alla posizione iniziale. Ė quello che accade all'acqua del mare elevata dalle nubi sulla cima delle montagne, che di là ricade lungo il fiume facendo lavorare il mulino.

Luniversalità del concetto dì energia ci suggerisce delle considerazioni di scala. Nella vita di tutti i giorni, prendiamo come unità di lavoro o energia una quantità che ci è familiare: come spostare un libro che ha una massa di 100 grammi (e perciò una forza-peso attorno a 1 newton) da uno scaffale alto 1 metro. Definiamo l'unità di energia joule (J) (dal nome dell'inglese Joule) come:

1 joule $=1$ newton $\mathrm{x} 1$ metro, cioè: $1 \mathrm{~J}=1 \mathrm{Nm}$

Invece a livello microscopico abbiamo introdotto l'eV. Orbene, il legame fra le due unità è circa: 


$$
1 \mathrm{~J} \sim 10^{19} \mathrm{eV}
$$

Cioè occorre spostare $10^{19}$ elettroni da un polo all'altro di una pila da 1 volt per fare andare un motorino che sollevi il libro di un metro. Tenuto conto che un liquido o solido ha $10^{22}$ atomi per $\mathrm{cm}^{3}$ (il volume contenuto in un cucchiaio) e che nella soluzione elettrochimica di una pila ogni molecola sciolta può fornire un elettrone, si vede che ce ne sono più che a sufficienza per fare il lavoro di 1 joule.

\subsection{Nota sulle unità di misura}

Conveniamo di prendere come unità di massa il kilogrammo $(\mathrm{kg})$, come unità di lunghezza il metro $(\mathrm{m})$ e come unità di tempo il secondo(s). La velocità sarà misurata in metri per secondo $\left(\mathrm{m} / \mathrm{s} \mathrm{o} \mathrm{ms}^{-1}\right)$; l'accelerazione, che è la velocita della velocità, in $\mathrm{m} / \mathrm{s}^{2} \mathrm{o} \mathrm{ms}^{-2}$; ne consegue che in base alla legge di Newton:

$$
\text { forza }=\text { massa } \mathrm{x} \text { accelerazione }
$$

l'unità di forza è quella che imprime l'unità di accelerazione all'unità di massa.

Essa è chiamata newton $(\mathrm{N})$ e vale:

$$
1 \mathrm{~N}=1 \mathrm{~kg} \cdot \mathrm{m} \cdot \mathrm{s}^{-2}
$$

In elettricità, si conviene di chiamare potenziale elettrico il lavoro per unità di carica e di misurarlo in volt $(\mathrm{V})$. Si conviene inoltre di chiamare campo elettrico la forza per unità di carica. Siccome la carica si misura in coulomb (C) ne segue:

$$
1 \mathrm{~V}=1 \mathrm{~J} / \mathrm{C}=1 \mathrm{JC}^{-1}
$$

Il campo elettrico è definito come una forza elettrica $(\mathrm{N})$ per unità di carica (cioè N/C), ma siccome abbiamo visto che $\mathrm{V}=\mathrm{J} / \mathrm{C}$ possiamo anche misurare il campo come potenziale per unità di spostamento, cioè indicando con [.] la misura:

$$
\text { [campo] }=\mathrm{N} / \mathrm{C}=(\mathrm{J} / \mathrm{m}) / \mathrm{C}=\mathrm{V} / \mathrm{m}=1 \mathrm{Vm}^{-1}
$$

Le grandezze per unità di lunghezza, o grandezze specifiche, dipendono ovviamente dallo spessore su cui vengono valutate. Una pila da $1 \mathrm{~V}$, fra i due elettrodi posti a $10 \mathrm{~cm}$, dà un campo di $10 \mathrm{~V} / \mathrm{m}$; lo stesso potenziale di $1 \mathrm{~V}$ applicato a una membrana biologica di spessore $1 \mathrm{~nm}=10^{-9} \mathrm{~m}$ dà un campo di $10^{9} \mathrm{~V} / \mathrm{m}$. 


\section{Particelle e forze}

Il successo della dinamica di Galileo-Newton fece nascere la speranza di poter descrivere tutta la realtà osservabile allo stesso modo: cioè come il moto di un punto geometrico (a cui attribuiamo una massa) in un paesaggio dell'energia. Il programma di riduzione al punto-massa ha funzionato per il libro, per il sistema Terra-Sole, per l'elettrone. Può funzionare sempre?

Il successo del modello newtoniano ne fece il modo universale di fare la fisica. Ciò implica una fede quasi religiosa in due concetti: determinismo e riduzionismo.

La dinamica di una particella è deterministica perché legge di forza e condizioni iniziali ne determinano il futuro. Il grafico della velocità di crescita di una variabile permette di stabilire come si comporta la variabile. Ora, data la forza, questa è proporzionale all'accelerazione che è la velocità della velocità: quindi possiamo conoscere come varia la velocità nel tempo a partire da una velocità iniziale. Nota la velocità, possiamo stabilire gli spazi percorsi a partire da una certa posizione iniziale. In conclusione, note forze, più velocità e posizione iniziale, sappiamo tutto.

Nella tradizione aristotelica, per cui scienza è "conoscenza delle cose attraverso le cause», si distinguevano quattro cause: materiale e formale (nel caso di un vaso di creta, rispettivamente la creta e la forma del vaso), efficiente (o motrice) e finale (rispettivamente, l'opera del vasaio che costruisce il vaso e lo scopo per cui il vaso è fatto). Orbene, vedremo che la ricerca delle particelle corrisponde alla ricerca della causa materiale, mentre la causa formale è stata sottovalutata dal riduzionismo, per cui la forma emerge automaticamente 'ammucchiando' i costituenti. Circa la causa efficiente, questa fu identificata con le forze e le condizioni iniziali che determinano il futuro. La causa finale fu considerata come un equivoco antropomorfico privo di rilevanza nell'indagine della natura.

Torniamo alla causa materiale. Se ora possiamo decomporre (ridurre) un corpo nei suoi costituenti, e ogni pezzo si comporta come la particella di Newton, sapremo tutto sul corpo. Se abbiamo una palla da biliardo, questa ci dà già l'idea del punto-massa di Newton; ma se abbiamo un fluido (gas o liquido) o un corpo 'molle', come un essere vivente, è meglio prima spezzare questo in porzioni più piccole per cui possa valere il programma di Newton. Questo programma di riduzione già presente $\mathrm{a}$ Newton (che faceva anche l'alchimista) diventò il programma della chimica, con Lavoisier.

$\mathrm{Si}$ arrivò a classificare i componenti elementari o molecole. In base ai rapporti degli ingredienti che entrano nelle reazioni chimiche (rapporti stechiometrici) si arrivò a stabilire che le molecole sono fatte di atomi (Dalton). Se prendiamo un numero di grammi pari al peso molecolare di una sostan$\mathrm{za}$, cioè - come si dice - una grammo-molecola o mole (per es. 2 grammi di idrogeno, o 18 grammi di acqua), vi è contenuto un numero fisso di molecole (numero di Avogadro), pari a circa $6 \cdot 10^{23}$, indipendentemente dalla sostanza.

Nel frattempo, si stabilivano le regole di attrazione e repulsione fra cariche elettriche. Dunque non c'era più solo la forza gravitazionale di Newton, ma an- 
che una forza che lega le particelle dotate di carica elettrica. Più tardi si stabilì che i fenomeni magnetici e ottici sono varianti delle forze elettriche, osservate in presenza di grandi velocità relative fra le cariche; si parla allora di fenomeni elettro-magnetici, che includono l'ottica, e di azioni elettrodinamiche.

Lo scambio di ingredienti nelle reazioni chimiche mostrava come le molecole fossero particelle composte. Si chiamarono atomi (cioè 'non-tagliabili') le particelle elementari di cui erano composte le molecole. Ovviamente il peso di una molecola è la somma dei pesi atomici dei componenti. Fatto uguale a 1 il peso dell'elemento più leggero (idrogeno), Mendeleev classificò tutti gli elementi noti in una tabella a due entrate. Si stabilì che dovevano esserci 92 elementi: non tutte le caselle corrispondevano ad elementi noti, ma quelli mancanti furono trovati successivamente.

La scoperta dell'elettrone, o particella elementare di carica negativa contenuta nell'atomo, fece supporre che un atomo neutro avesse un ugual numero di cariche positive per compensare gli elettroni (i quali si pensavano immersi nell'atomo come i canditi nel panettone). Quando un atomo perde uno (o più) elettroni diventa uno ione positivo. Tutte le proprietà elettriche delle soluzioni sono spiegate in termini di ioni ed elettroni. Lo ione più elementare è costituito dall'atomo di idrogeno, quando perde il suo elettrone ed è chiamato protone.

La scoperta della radioattività (Becquerel e Curie) mostrò che gli atomi trasmutano, cioè non sono oggetti elementari ma possono acquistare o perdere dei pezzi, cambiando posto nella tabella di Mendeleev.

Il bombardamento di atomi con micro-proiettili portò Rutherford a scoprire che l'atomo non è un panettone, ma un sistema solare in miniatura con gli elettroni distribuiti come pianeti, un enorme spazio vuoto, e un nucleo, dove sono concentrate le cariche positive, che è $10^{4}$ volte più piccolo del raggio dell'atomo. Si scoprirono poi particelle neutre con il peso del protone: i neutroni. Si può capire così perché il peso atomico, rispetto all'idrogeno come unità, cresca più rapidamente del numero atomico (cioè del numero di cariche elettriche positive): ciò è dovuto all'aggiunta dei neutroni.

Ma come sta assieme tutto ciò? Conosciamo la forza di gravitazione, che tiene assieme i corpi celesti. Conosciamo le forze elettriche, che attraggono cariche di segno contrario. Dobbiamo postulare che esistono forze nucleari che tengono vicini, ad esempio, i due protoni di un nucleo di elio in modo più forte della repulsione elettrica fra le due cariche positive. Queste forze sono scambiate anche tra protoni e neutroni, cioè tra tutti i componenti del nucleo o nucleoni.

Finché l'energia di legame cresce (cioè dall'idrogeno al ferro) sono favoriti i processi di fusione; per nuclei pesanti invece sono favoriti i processi di scissione (o fissione). Le energie di legame sono sui $5 \mathrm{MeV}$ (mega eV, cioè $10^{6} \mathrm{eV}$ ) per nucleone, quindi dato un nucleo con qualche decina di nucleoni, se lo si bombarda con proiettili di energia di alcune centinaia di $\mathrm{MeV}$, si dovrebbero liberare protoni e neutroni non più legati. Invece con lo sviluppo delle tecniche di accelerazione, andando a molte centinaia di MeV, si evidenziarono particelle esotiche e non semplicemente protoni o neutroni a grande energia cinetica. Ciò sembrava riportare la situazione alla chimica 
del Settecento. L'evidenza sperimentale ha mostrato che protoni, neutroni e le altre particelle esotiche sono in effetti particelle composte di quarks.

Oltre alle tre forze suddette, nei processi nucleari di fusione fra nuclei leggeri nelle stelle gioca un ruolo fondamentale la forza debole.

Le forze sono mediatori fra particelle. Per Newton ciò avveniva mediante azioni a distanza che istantaneamente giungono dal Sole alla Terra. Invece la fisica dell'Ottocento mostrò che le azioni sono contigue, fra posizioni spaziali vicine. Nessun oggetto può spostarsi a velocità maggiore della luce, perciò le azioni contigue implicano una velocità limitata di propagazione. Se per ipotesi il Sole scomparisse istantaneamente, solo dopo otto minuti l'effetto si sentirebbe sulla Terra. Parleremo di campi di forza (Faraday) per indicare che la forza esiste in ogni parte dello spazio, come si può vedere con la limatura di ferro per i campi magnetici. Se fra Terra e Sole non c'è un'azione istantanea, ma un campo che si propaga a velocità finita e che riempie tutti i punti dello spazio, quali sono le modalità di propagazione? Lo vedremo nella sezione successiva.

Intanto, è importante notare che le forze gravitazionali ed elettriche producono i loro effetti anche a grandi distanze, mentre le forze nucleari e deboli agiscono solo a distanze sui $10^{-12} \mathrm{~cm}$ : è perciò che non erano note prima che la sperimentazione risolvesse queste distanze sub-atomiche.

\section{Onde}

La mente umana, nel fare schemi del mondo, si dibatte fra discreto e continuo. Così la matematica, fin dagli inizi, si differenziò in aritmetica (sistema numerico discreto) e geometria (linee continue). Questo dilemma è sempre vivo, e accanto alla ricerca di particelle c'è sempre stato il tentativo di dissolverle in un continuo: da Leibniz che criticava il meccanicismo cartesiano, ai modelli che cercano di spiegare le particelle come singolarità dei campi.

Ora, tutti abbiamo l'idea delle onde (ad esempio sulla superficie dell'acqua). Possiamo, con una foto istantanea, rappresentare l'onda nello spazio (Fig. 40a), oppure, ponendoci in una posizione fissa, misurare come l'onda sale e scende nel tempo (Fig. 40b). In corrispondenza, si possono definire le grandezze ivi indicate. Si noti che l'onda è positiva o negativa rispetto alla posizione di quiete. La velocità a cui la cresta dell'onda si vede muoversi è data da spazio diviso tempo, cioè lunghezza d'onda diviso periodo, o moltiplicato frequenza:

\section{velocità d'onda = lunghezza d'onda $\mathrm{x}$ frequenza}

Si noti che l'onda corrisponde al passaggio di una perturbazione, non al moto fisico di particelle. Se in un porto abbiamo molti tappi galleggianti in fila, e arriva un'onda, i tappi vanno su e giù, ma rimangono nella stessa posizione. Se osserviamo come si muove la cresta, questa 'salta' da tappo a tappo con la velocità d'onda. 
Le onde sono longitudinali se la perturbazione è nella stessa direzione in cui si propaga l'onda (come l'aria mossa da un tamburo) o trasversali se la perturbazione è perpendicolare alla propagazione, come nel caso dei tappi. A differenza delle particelle che si sommano sempre se ammucchiate nello stesso posto, due onde possono sommarsi ma anche distruggersi. Si dice che interferiscono: il risultato dipende dalla posizione mutua delle creste che si chiama fase relativa (Fig. 41).

a)

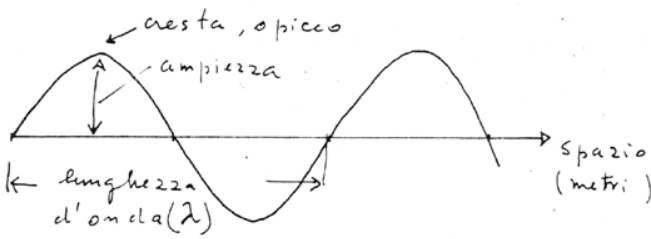

b)

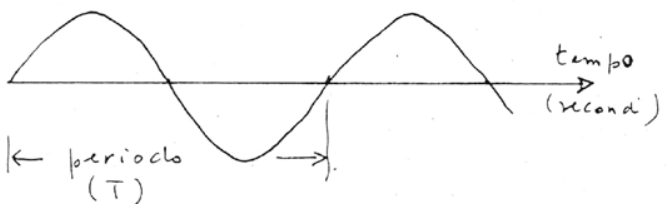

Figura 40 -a) Onda nello spazio. Si ripete periodicamente dopo una lunghezza d'onda (che si indica di solito con $\lambda$ ). b) Onda nel tempo. Il periodo (o ciclo) si indica con $\mathrm{T}$; l'inverso $\mathrm{v}=1 / \mathrm{T}$ corrisponde a quanti periodi ci sono in un secondo: si chiama frequenza. L'unità di frequenza è 1 /secondi ed è detta hertz.

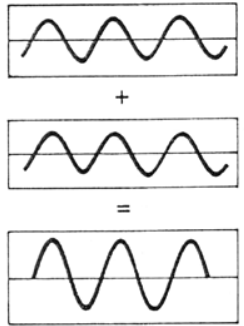

(a)
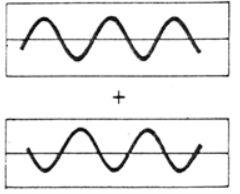

$=$

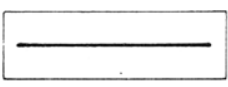

(b)

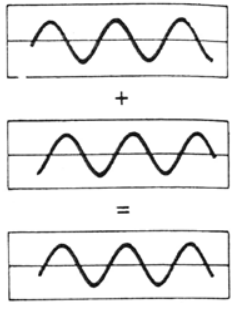

(c)

Figura 41 - Interferenza fra due onde: a) fase relativa nulla: si ha interferenza costruttiva; b) fase pari a mezza lunghezza donda: interferenza distruttiva; c) fase qualunque: interferenza parziale.

Fu appunto per l'evidenza di interferenza che Huygens sviluppò l'idea che i fenomeni luminosi siano dovuti a propagazioni di onde, e non a flusso di particelle come credeva Newton.

Un altro fenomeno importante delle onde è la diffrazione (cioè lo spezzarsi dei cammini rettilinei di propagazione), descritto da Grimaldi nel 
Seicento, e che consiste nel vedere luce dove si aspetta ombra, dietro un forellino illuminato dal Sole. L'effetto di diffrazione si spiega meglio con riferimento alle onde dell'acqua che incontrano ostacoli (Fig. 42).
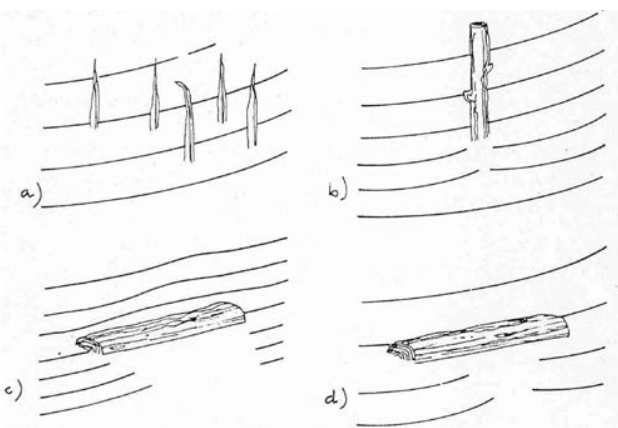

Figura 42 -Onde in acqua che passano fra oggetti di dimensioni differenti: a) fili d'erba; b) bastone; c) tronco di lunghezza grande rispetto alla lunghezza donda; d) tronco di lunghezza piccola rispetto alla lunghezza d'onda. Si noti che la regione d'ombra appare definita solo per lunghezze d'onda piccole rispetto alloggetto. Ciò spiega i limiti risolutivi di un'ispezione ottica sugli oggetti piccoli. Quando l'oggetto diventa confrontabile con la lunghezza d’onda della luce con cui lo si illumina, la sua ombra non è più ben definita. Possiamo vedere 'bene' solo dettagli grandi rispetto alla lunghezza d'onda.

Fra l'altro il fenomeno di diffrazione suggerisce che noi perdiamo i dettagli degli oggetti quando li osserviamo mandandoci contro un'onda di lunghezza pari o maggiore alle dimensioni dell'oggetto. Per riassumere lo stato di quanto sappiamo sui fenomeni luminosi, in figura 43 si riporta lo spettro delle onde elettromagnetiche, con i vari nomi che le regioni prendono. Si sono segnate sia le lunghezze d'onda sia le frequenze. Si noti quanto è limitata la regione visibile, in cui i nostri occhi vedono colori, rispetto alle regioni a grande lunghezza d'onda (infrarosso e oltre) e a piccole lunghezze d'onda (ultravioletto e oltre).

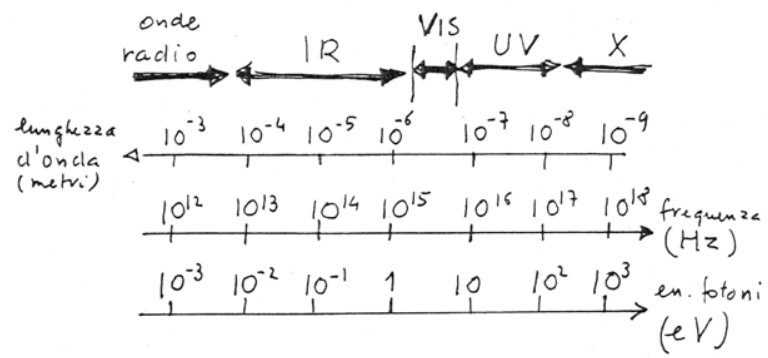

Figura 43 - Lo spettro elettromagnetico. Riportiamo tre scale: lunghezze d'onda (in metri), frequenze (in periodi al secondo o hertz) ed energie dei fotoni (in eV). Si noti la proporzionalità inversa: la prima scala cresce verso sinistra, la seconda e terza crescono verso destra. IR, Vis e UV indicano rispettivamente infrarosso, visibile e ultravioletto. 


\section{Bilanci energetici: chimica e vita}

La fisica moderna ha scoperto che la luce non è solo onda, ma la sua energia è concentrata in corpuscoli o granelli (quanti ofotoni) la cui energia dipende dal colore, cioè dalla frequenza. Se disponiamo i colori secondo l'arcobaleno - rosso-giallo-verde-blu-violetto -, spostandoci verso destra cresce la frequenza e cresce anche l'energia del singolo quanto, da $2 \mathrm{eV}$ a $4 \mathrm{eV}$, secondo la proporzionalità:

\section{energia del fotone $=h \times$ frequenza}

dove, attraverso la costante universale $h$ (detta di Planck), la frequenza dell'onda è tradotta in energia di ciascuno dei granelli (o fotoni)che costituiscono l'onda.

Un fotone può interagire con un singolo atomo o molecola cedendo la sua energia in un processo di assorbimento; viceversa un atomo in uno stato eccitato (cioè che abbia ricevuto energia in più rispetto al suo stato di riposo) può cedere questa energia al campo elettromagnetico emettendo un fotone.

La molecola fotosensibile come la rodopsina (che è alla base dei processi fotochimici nella retina dell'occhio che danno luogo alla visione) ha due valli di energia A e B (Fig. 44). Normalmente essa è situata in A. Nella figura, IR (infrarosso) indica un fotone con energia al disotto del visibile, UV invece ne indica uno al disopra.

Per spostarsi da $A$ in $B$ occorre fornire un fotone con energia superiore alla barriera da superare. Se le valli $A$ e $B$ fossero allo stesso livello, nel passare da $A$ a $B$ non si guadagnerebbe energia; ma $B$ è più alto di $A$. Se la molecola viene colpita da un fotone intermedio fra $2 \mathrm{eV}$ (rosso) e $4 \mathrm{eV}$ (violetto), essa può passare da $A$ a $B$ e l'eccesso di energia viene utilizzato per mandare un impulso elettrico sul nervo ottico. Se invece mandiamo fotoni IR, cioè più piccoli di $2 \mathrm{eV}$, non si riesce a superare la barriera tra $A$ e $B$.

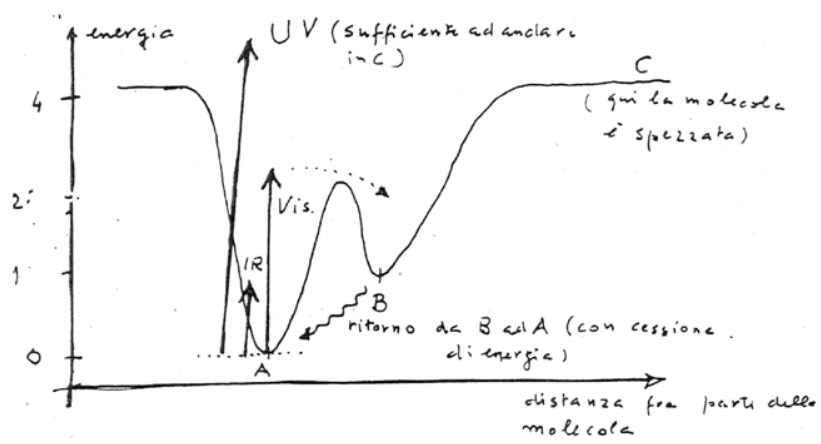

Figura 44 - Paesaggio dell'energia per molecola fotosensibile. La condizione di riposo è A. Lenergia dei fotoni (IR, Vis, UV) è indicata da frecce crescenti. Il fotone Vis permette il salto da $\mathrm{A} \mathrm{aB}$. Da $\mathrm{B}$ si torna ad $\mathrm{A}$ cedendo energia come attività elettrica della retina. 
Domanda: accumulando 10 fotoni piccoli da $0,3 \mathrm{eV}$, è possibile ottenere l'effetto che si otterrebbe con un fotone grande da $3 \mathrm{eV}$ ?

Risposta: No! Di solito solo un fotone per volta può interagire con una molecola. I fotoni IR si limitano ad agitare la molecola intorno ad $A$, cioè a scaldarla. Anche scaldare-agitare le molecole attorno ad $A$ vuol dire cedere energia, c'è perciò una 'traduzione' da energia a temperatura, precisamente una energia di l e V scalda una molecola di circa 10.000 gradi Kelvin, cioè:

$$
1 \mathrm{eV} \approx 10^{4} \mathrm{~K}
$$

Al contrario, un fotone UV - per esempio da $10 \mathrm{eV}$ - permette alla molecola non solo di superare la barriera ma di andare oltre $C$ : ciò vuol dire che la distanza fra parti della molecola diventa grandissima, cioè la molecola si spezza in due tronconi (si dissocia). L'UV è dannoso per le molecole. Orbene, in base a quanto visto, i fotoni Vis sono i migliori per la chimica delle molecole: né troppo caldi (UV), né freddi (IR).

In figura 44 si è mostrata una reazione chimica endotermica in cui $A$ (stato iniziale) è più basso di $B$ : per spostarsi in $B, A$ richiede energia dalla luce. Quanto avviene nei fotosensori (rodopsina) ha luogo anche nei fotoutilizzatori delle piante (clorofilla). L'energia è fornita da un fotone che permette alla molecola di essere promossa da A in B. Il salto di energia viene poi utilizzato nella sintesi clorofilliana.

La figura 45 mostra perché una stella come il Sole può assicurare la vita. Dare poca energia non basterebbe alla chimica della vita, perché non si supera la barriera fra due stati; troppa energia nuoce alla molecola(la dissocia). La vita sulla Terra può svilupparsi perché il nostro Sole è nel giusto mezzo.

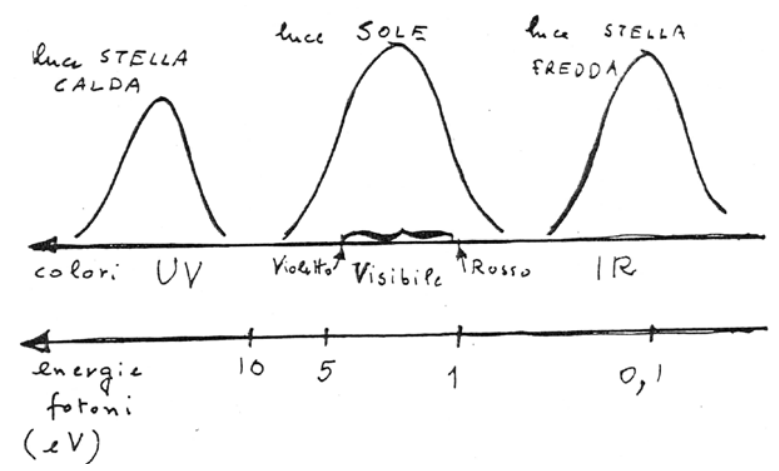

Figura 45 - Spettri di emissione del Sole e di altre due stelle (calda e fredda) per mostrare come solo stelle come il Sole permettono reazioni chimiche, e perciò la vita. 


\subsection{Chimica del vivente e chimica industriale}

Chiamiamo reazioni esotermiche quelle in cui invece lo stato finale ha energia più bassa dello stato iniziale (Fig. 46)e la differenza di energia $E_{A B}$ può essere utilizzata (combustione). Nei processi di metabolismo (chimica del vivente) le molecole cadendo da $A$ a $B$ cedono energia. Prima però devono superare la barriera $C$ con l'aiuto dell'enzima (come descritto nella didascalia della figura).

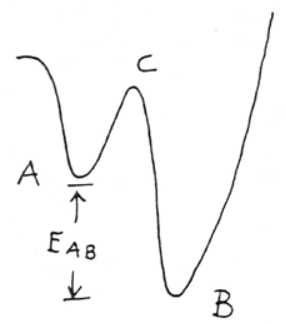

$\mathrm{E}_{\mathrm{AB}}=$ salto di energia

utilizzabile per fare

lavoro

Figura 46 - Reazione esotermica: la molecola passando da A a B può fare lavoro. Ma prima deve vincere la barriera $\mathrm{AC}$ che può essere più alta dei fotoni IR disponibili a temperatura ambiente. Si passerà da $\mathrm{A}$ a $\mathrm{B}$ o scaldando a una temperatura che favorisca il salto a $\mathrm{C}$ (reazioni chimiche industriali) o deprimendo la barriera $\mathrm{C}$ con l'aiuto di una molecola ausiliaria che non si consuma nel processo (catalizzatore, detto enzima nelle reazioni biologiche).

Nella chimica biologica, gli enzimi permettono di superare la barriera tra le due valli 'a freddo', cioè alla temperatura corporea $\left(37^{\circ} \mathrm{C}=300 \mathrm{~K}\right)$. Nella chimica industriale, invece, la molecola deve essere scaldata fino a migliaia di gradi per scavalcare la barriera (ricordiamo che $1 \mathrm{eV}=10^{4} \mathrm{~K}$, e per attivare una combustione occorre circa $0,1 \mathrm{eV}$ cioè un migliaio di gradi $\mathrm{K}$ (pari a circa $700{ }^{\circ} \mathrm{C}$ ).

\section{I quanti}

\subsection{Il corpo nero e l'effetto fotoelettrico}

L'unificazione fra fenomeni elettrici, magnetici e ottici operata da Maxwell (1866) fa sì che si possa spiegare la luce emessa da un atomo come un'onda elettromagnetica causata dall'avvicinarsi e allontanarsi delle cariche positive e negative nell'atomo; la radiazione è cioè dovuta all'accelerazione delle cariche. In ciò un atomo non differisce dall'antenna di una radio (Fig. 47). L'antenna emette un'onda di frequenza pari alla frequenza con cui vibrano le sue cariche elettriche. Se nell'antenna coesistono frequenze diverse, l'antenna emetterà i vari 'colori' corrispondenti. 


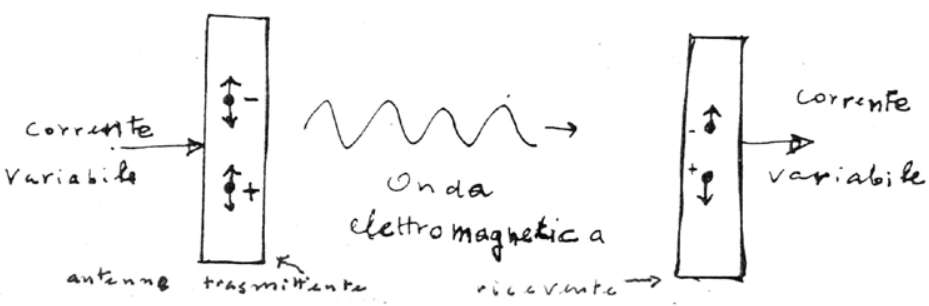

Figura 47 - Come avviene la conversione da corrente elettrica (provocata per esempio da un microfono) a onda elettromagnetica, e la riconversione da onda a corrente (che, nell'esempio, farà vibrare l'altoparlante). Si noti che in trasmissione la vibrazione fra cariche è provocata dalla corrente e genera l'onda; in ricezione la vibrazione è provocata dallonda e genera la corrente.

Se ora pensiamo che i moti delle cariche differiscono da atomo ad atomo, ci aspettiamo che ogni atomo emetta (in trasmissione) o assorba (in ricezione) una particolare lunghezza d'onda, cioè uno particolare colore. In effetti gli spettri di emissione e assorbimento sono specifici di ciascuna specie atomica, come le impronte digitali per gli individui.

Tutto ciò vale purché, per restare confinati alla fisica dell'ordine, gli atomi siano 'diluiti' (gas tenue) in modo da potersi considerare come individui isolati. Se invece li impacchettiamo in modo che si influenzino a vicenda con urti, allora passiamo alla fisica del disordine. I moti disordinati non daranno più colori precisi: è come se esistessero antenne in grado di emettere onde disparate su molte frequenze. Si avrà allora uno spettro che non dipende più dagli individui, ma solo dalla temperatura a cui gli individui sono stati portati. Questo spettro è universale, e si chiama spettro di corpo nero (per analogia con l'interno di un forno di cui si scaldino le pareti finché queste si arroventino emettendo luce; se il buco di osservazione è piccolo, il forno perde poco verso l'esterno e perciò se inviamo luce sull' ingresso, praticamente nulla torna fuori, per cui il nome nero).

La figura 48 mostra la 'densità' di energia irraggiata da un corpo nero a una certa temperatura. Chiamiamo densità il quoziente fra energia e intervallo di frequenze su cui è stata emessa. Per avere l'energia totale, dobbiamo sommare tutti i contributi di densità, moltiplicandoli per il corrispondente intervallo di frequenze. Chiariamo questa idea:

$$
\text { densità }=(\text { energia emessa }) / \text { frequenza }
$$

Perciò: 
Uno di questi addendi della somma è tratteggiato in figura e come si vede è un'area. Per avere tutta l'energia dobbiamo perciò valutare tutta l'area compresa fra la curva e l'asse orizzontale.

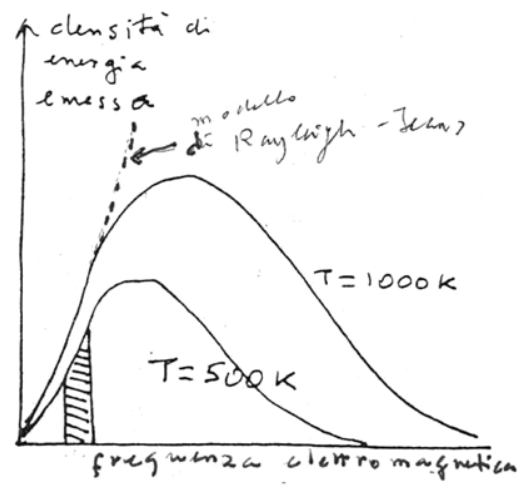

Figura 48 - Spettro di emissione del corpo nero, cioè densità di energia irraggiata da un corpo portato ad una temperatura T. Due esempi a temperatura diversa; curva tratteggiata $=$ teoria di Rayleigh e Jeans.

Ora la fisica classica dice che quando un oggetto è a temperatura T, qualunque sua vibrazione riceve lo stesso rifornimento di energia, un 'boccone' proporzionale a T indipendentemente dalla natura degli atomi che compongono l'oggetto. Ma il numero di vibrazioni diverse cresce con la frequenza e perciò, se ciascuna contribuisce con lo stesso boccone di energia proporzionale a $\mathrm{T}$, si avrebbe una densità come quella tratteggiata in figura, con un'area sottostante (energia totale irraggiata) infinita! Per giustificare la curva sperimentale occorre rinunciare all'ipotesi che ogni vibrazione possa avere la stessa energia, dipendente solo da $\mathrm{T}$ e indipendente dalla sua frequenza.

Max Planck introdusse nel 1900 l'ipotesi che, pur essendo le onde elettromagnetiche un continuo, l'energia che esse scambiano con le cariche, cioè con gli atomi della parete del forno, sia 'granulare', sia fatta cioè di zollette distinte ognuna delle quali (quanto di energia) è proporzionale alla frequenza dell'onda con una costante di proporzionalità chiamata $h$.

Dunque l'ipotesi di Planck è:

\section{quanto di energia $=h \times$ frequenza}

Questa ipotesi porta a una legge di emissione che riproduce quella sperimentale, eliminando il paradosso dell'energia totale infinita.

Ma cosa significa che l'energia elettromagnetica si propaga a quanti? Lo vediamo subito considerando l'effetto fotoelettrico (Fig. 49). La corrente di elettroni emessi da un metallo, quando questo è investito dalla luce, è nulla per certi colori, cioè a grande lunghezza d'onda (ad es. il rosso) an- 
che se illuminiamo con grandi intensità di luce; la corrente è non nulla se spostiamo il colore verso il blu. La spiegazione di Einstein (1905) è la seguente: i quanti di energia luminosa non si possono sommare, ma agiscono individualmente: uno per ogni elettrone. Per superare l'energia attrattiva che trattiene gli elettroni nel metallo, occorre che ogni elettrone riceva un quanto di energia sufficiente.

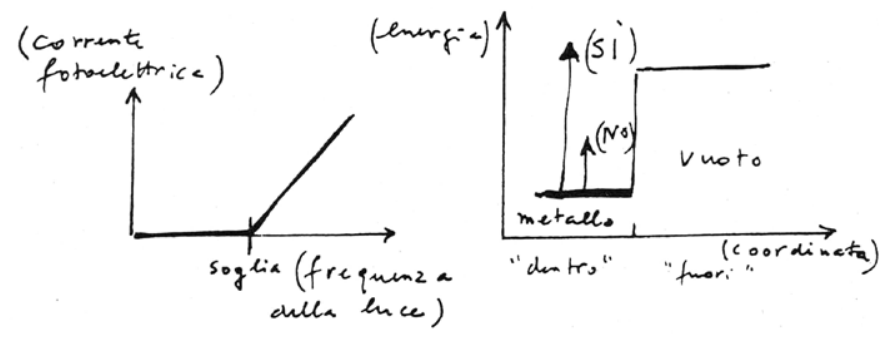

Figura 49 - Effetto fotoelettrico: la corrente degli elettroni emessi da un metallo illuminato da luce è nulla finché la luce non ha una frequenza superiore ad un valore di soglia. Spiegazione di Einstein: emettere elettroni vuoi dire superare uno scalino di energia fra dentro e fuori del metallo. Il quanto luminoso, o fotone, deve avere unenergia pari almeno allo scalino.

Dunque, Planck ed Einstein hanno introdotto una dualità onda-corpuscolo: la luce, che negli esperimenti di diffrazione e interferenza si comporta come onda, va considerata come un insieme di granelli (i fotoni) per riuscire a spiegare il corpo nero e l'effetto fotoelettrico.

\subsection{L'atomo di Bohr}

Abbiamo visto che l'atomo è come un sistema solare. Ma allora l'elettrone legato a un nucleo atomico dovrebbe emettere un'onda, in quanto è accelerato nel suo moto orbitale; pertanto, deve perdere energia, e perciò cadere a spirale sul nucleo. Invece esistono gli atomi stabili! Per spiegare ciò, Niels Bohr postulò nel 1913 che l'elettrone non può assumere un continuo di valori di energia, come la pallina sul piano inclinato, ma solo alcuni valori permessi, come in una scala a pioli. I livelli permessi di energia furono calcolati come segue: l'elettrone che si muove con velocità $v$ ha un impulso $m v$ (prodotto di massa per velocità).

L'impulso è la quantità scambiata negli urti. Ad esempio si può ricavare un impulso dannoso o urtando un oggetto pesante a bassa velocità (investimento da un camion) o urtando un oggetto leggero ma ad alta velocità (un proiettile di pistola). Orbene, Bohr collegò l'impulso dell'elettrone con il raggio dell'orbita permessa (e conseguentemente, con l'energia dell'orbita) mediante una relazione di proporzionalità inversa che contiene la costante $h$ di Planck: 


\section{circonferenza orbita $=2 \pi \cdot$ raggio $=n$ volte $\cdot h /$ impulso}

Sono permesse solo quelle orbite $n=1,2,3 \ldots$ con raggi e impulsi tali da soddisfare la relazione di Bohr. Ad esse corrispondono le energie discrete $E_{1}, E_{2} \ldots$ Le altre energie sono proibite. Dunque l'atomo è stabile solo a questi livelli di energia, $E_{1}, E_{2} \ldots$ e quando scende (o sale) da un livello ad un altro allora emette (o assorbe) un fotone pari al salto di energia (Fig. 50).
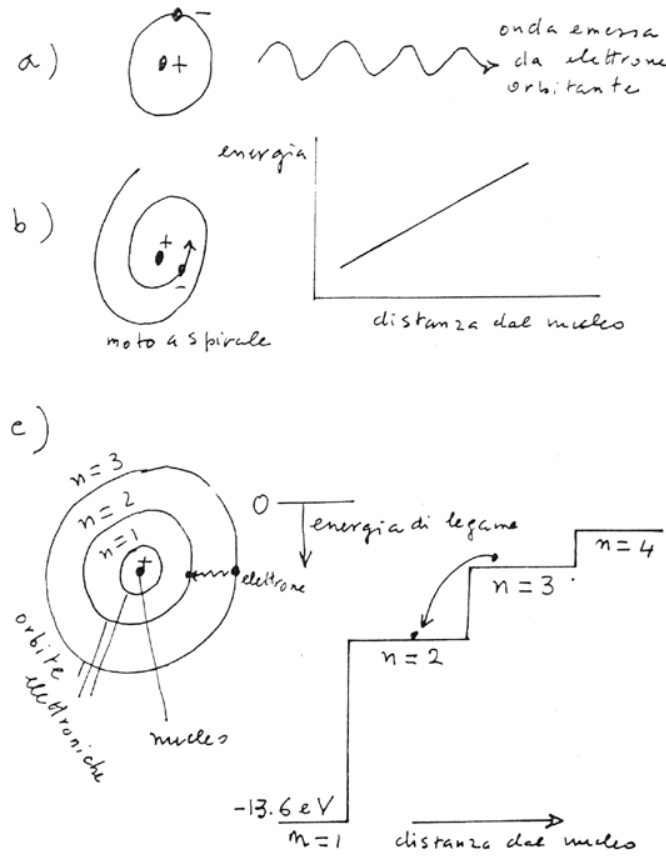

Figura 50 -a) Atomo di Rutherford: l'elettrone irraggia un'onda. b) Di conseguenza perde energia con continuità e cade sul nucleo. c) Ipotesi di Bohr: l'elettrone ha solo alcuni valori permessi d'energia, e tutti gli altri sono proibiti (livelli quantizzati di energia). Solo quando salta fra due livelli cè emissione; quando permane su unorbita non irraggia (al contrario del caso classico a).

Questa relazione misteriosa fra raggio atomico e impulso dell'elettrone fu spiegata da Louis De Broglie (1922) invocando una dualità corpuscoloonda. Come la luce è non solo onda, ma anche quanti, così l'elettrone è non solo particella ma anche onda, con lunghezza d'onda $\lambda$ tale che:

$$
\lambda=h / i m p u l s o
$$

Vediamo come il fenomeno di interferenza spieghi l'atomo di Bohr. Quando cerchiamo di confinare un'onda fra due specchi, ci riusciamo 
solo se la distanza fra i due specchi è pari a un numero intero di mezze lunghezze d'onda, altrimenti l'onda diretta e l'onda riflessa si cancellano per interferenza (Fig. 51a). Ripetendo lo stesso discorso per un'onda che si chiude su se stessa su un cerchio, vediamo che essa può esistere solo quando la circonferenza è pari ad un numero intero di lunghezze d'onda. Pertanto dalla ipotesi di De Broglie discende la condizione di Bohr (Fig.51b).
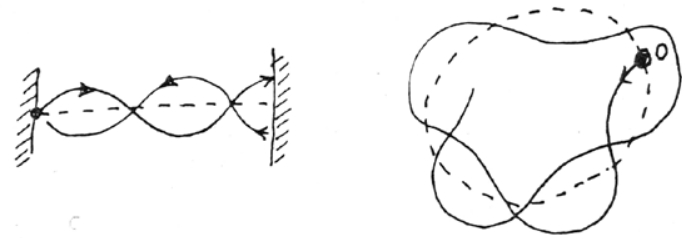

INTERFERENZA
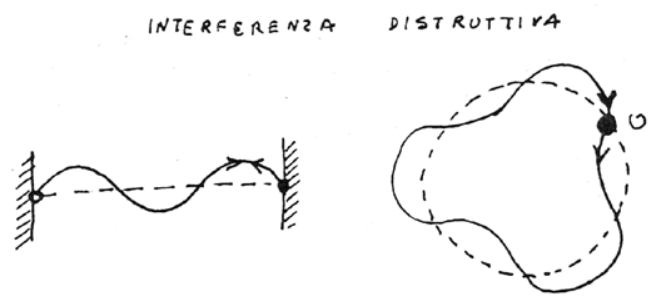

INTERFERENZA

COSTRUTTIVA

a)

b)

Figura 51 - a) Londa lanciata dallo specchio a sinistra si riflette sullo specchio a destra; l'ampiezza riflessa è uguale e di segno contrario a quella incidente, in quanto la somma delle due deve dare ampiezza zero, visto che l'onda non può penetrare nello specchio; pertanto, onda diretta e riflessa si distruggono per interferenza. Solo se $\lambda / 2$ è contenuta in L un numero intero di volte (figura in basso), cioè se l'onda ha ampiezza nulla su entrambi gli specchi, si può mantenere unonda stazionaria. b) Lo stesso, ma avvolgendo il percorso su una circonferenza. Condizione per unonda stabile: $\lambda$ deve essere contenuta un numero intero di volte nella circonferenza.

\subsection{I successi della fisica quantistica}

La meccanica dei processi microscopici in cui le onde sono anche corpuscoli (Planck) e le particelle anche onde (De Broglie) è stata chiamata meccanica quantistica. Abbiamo visto nella storia della scienza la dialettica fra continuo (geometria) e discreto (aritmetica). Ebbene, le ipotesi di Planck e De Broglie sembrano armonizzare questi due punti di vista contrastanti. Sia la struttura discreta degli spettri emessi dagli atomi, sia la stessa costruzione della tabella degli elementi atomici, con il ripetersi periodico delle proprietà chimiche, sono spiegate da questo riempimento successivo di un'orbita atomica con una, due, tre lunghezze d'onda. 
Ma se c'è un'onda associata ad ogni particella, la particella dovrebbe dar luogo a fenomeni di interferenza. Ripetiamo allora per un fascio di elettroni l'esperimento fatto da Young nel 1810 per le onde luminose.

L'esperimento dimostra che c'è veramente un'onda associata, la cui lunghezza d'onda si scala come previsto da De Broglie. Infatti, variando la velocità del fascio di elettroni (e pertanto l'impulso) si sposta il sistema di picchi e valli. Si noti l'enorme rilevanza applicativa di questo fatto. Quando con la luce non riusciamo più a vedere oggetti molto piccoli (come in biologia) perché la lunghezza d'onda luminosa è maggiore dell'oggetto (diffrazione), allora illuminiamo con fasci di particelle (elettroni o neutroni) e costruiamo perciò microscopi elettronici o neutronici.

Ricordiamo che la luce visibile ci permette di risolvere fino a $0,5 \mathrm{mi}$ crometri; invece con i microscopi a particelle possiamo risolvere fino a pochi angstrom (unità di misura pari a $10^{-10} \mathrm{~m}$, cioè a un raggio atomico).

\subsection{Le difficoltà concettuali della meccanica quantistica}

Abbiamo risolto i problemi che sembravano paradossali per la fisica classica (corpo nero, effetto fotoelettrico, stabilità degli atomi) $\mathrm{ma}$ al prezzo di rendere elusivi gli oggetti microscopici. Le onde luminose, in certi casi, si comportano come corpuscoli (fotoni); le particelle, in certi casi, si comportano come onde (regola di Bohr ed esperimenti di interferenza). Ma allora, che cosa sono? La difficoltà è che siamo in possesso di un formalismo, la meccanica quantistica, estremamente efficace per descrivere esperienze su scala atomica ma la cui interpretazione è ambigua. In effetti, se ci domandiamo che cosa siano gli enti che determinano le nostre misure, stiamo uscendo dalla logica della fisica, che consiste nel connettere risultati di misure per fare previsioni, per domandarci che cosa c'è dietro l'apparato di misura.

Potremmo dire che le idee di particelle e onde vengono dalle nostre esperienze di tutti i giorni, idealizzate con i concetti di discreto e continuo, e non è detto valgano per oggetti che non vediamo ma che percepiamo solo attraverso apparati di misura. Bohr espresse nel 1928 questa situazione di disagio con il cosiddetto principio di complementarità: un oggetto atomico o sub-atomico non è solo particella (come le palle di biliardo), né solo onda (come le onde nell'acqua). A seconda delle misure che si fanno, si evidenzierà una o l'altra caratteristica. Si noti che, per effetto di questa dualità, non possiamo determinare 'con precisione' e simultaneamente due grandezze quali posizione e impulso di un oggetto microscopico. Infatti, per misurare con precisione la posizione, dobbiamo guardare la particella con un'onda di lunghezza d'onda piccolissima, i cui fotoni trasferiscono un impulso grandissimo. Viceversa, se vogliamo determinare con precisione la velocità, dobbiamo vedere quanto tempo ci vuole ad attraversare una certa distanza L; ma dentro L non possiamo localizzare la particella, perché altrimenti l'atto di osservazione ne altererebbe la velocità! Questa impossibilità di misura simultanea precisa si chiama principio di indeter- 
minazione di Heisenberg (1927). Esso stabilisce che il prodotto delle due incertezze è uguale (o maggiore) alla costante $h$. Dato che il prodotto si deve mantenere costante, se un fattore diminuisce l'altro deve crescere.

La relazione di Heisenberg pone un limite di principio alla precisione della nostra misura.

\section{Spazio-tempo da Newton ad Einstein: le onde gravitazionali}

La struttura dello spazio-tempo è un concetto che si è evoluto con il progredire delle conoscenze scientifiche. Inizialmente il tempo era concepito come assoluto: Aristotele lo definiva come «la misura del movimento». Questa visione è stata ripresa e sviluppata da Newton: lo spazio e il tempo erano grandezze «assolute», indipendenti dalla natura e dal moto della materia. Secondo Newton, lo spazio costituiva una specie di griglia immutabile in cui avvenivano i fenomeni fisici. Lo spazio, inoltre, era regolato dalla geometria euclidea nella quale la distanza tra due punti costituisce un invariante fondamentale. Il tempo, o meglio gli intervalli di tempo, erano una grandezza misurabile, esattamente come la lunghezza di un oggetto.

Questa visione, accettabile per fenomeni in cui i corpi si muovono a velocità molto più piccole della luce, fu scardinata da Einstein (1905) con la teoria della relatività ristretta. Fino ad allora si riteneva che le leggi della meccanica non dovessero dipendere dal moto di chi le osservava, purché il moto fosse uniforme. Questa invarianza per trasformazioni da un sistema inerziale ad un altro è nota come relatività galileiana (cfr. Dialogo sopra i due massimi sistemi del mondo del 1632). Tuttavia le leggi dell'elettromagnetismo di Maxwell non erano invarianti per trasformazioni galileiane. Einstein ipotizzò che tutte le leggi fisiche dovessero essere le stesse in ogni sistema di riferimento inerziale, e che la velocità della luce fosse sempre la stessa, indipendentemente dalla velocità del sistema inerziale in cui la si osserva.

Con ciò Einstein pose le basi della teoria della relatività ristretta. Questo comportava una nuova definizione delle variabili spazio e tempo, non più assolute, ma relative all'osservatore che le misura, e una rappresentazione dei fenomeni fisici in uno spazio non più tridimensionale ma quadridimensionale (tempo $=$ quarta dimensione). I punti dello spazio-tempo sono detti eventi e ciascuno di essi corrisponde ad un fenomeno che si verifica in una certa posizione spaziale e in un certo momento. Ogni evento è perciò individuato da quattro coordinate. Quindi, la struttura dello spazio-tempo dipende dall'osservatore: in particolare, la distanza spaziale o temporale tra due eventi risulta diversa a seconda che l'osservatore che la misura sia fermo oppure in movimento rispetto agli oggetti osservati. Questo effetto è molto piccolo nella vita quotidiana, ma diventa importante per quegli oggetti che si muovono a velocità vicine a quella della luce. Le misure sperimentali della velocità della luce confermarono l'ipotesi di 
Einstein. Viene meno il concetto di spazio e tempo assoluti e separati l'uno dall'altro e si rimpiazza con il concetto di spazio-tempo.

Una seconda rivoluzione (1915), ad opera di Einstein, è la teoria della relatività generale, secondo cui la natura dello spazio-tempo è connessa con la quantità della materia presente. Lo spazio-tempo viene curvato dalla presenza di una massa, generando quello che viene definito un 'campo gravitazionale'. Questo effetto è reciproco ('la materia dice allo spazio come curvarsi, e lo spazio dice alla materia come muoversi') e matematicamente è descritto dalle 'equazioni di Einstein' consistenti nella relazione:

$$
\text { curvatura dello spazio }=\frac{\text { densità di massa }}{\text { energia della materia }}
$$

Si raffigura questa situazione come una palla che deforma un telo elastico teso con il suo peso, mentre un'altra pallina viene accelerata da questa deformazione e, in pratica, attratta dalla prima (Fig. 52). Ad essere deformato è lo spazio-tempo e non solo le dimensioni spaziali. Ogni oggetto presente nell'Universo influisce sullo spazio-tempo che lo circonda: per esempio, la Terra, con la sua massa, curva lo spazio circostante (generando la ben nota attrazione gravitazionale) e influisce sul tempo attraverso un rallentamento del ritmo di orologi identici posizionati a distanze decrescenti dal suo centro (come verificato dal confronto di orologi atomici posti a bordo di aerei o satelliti).

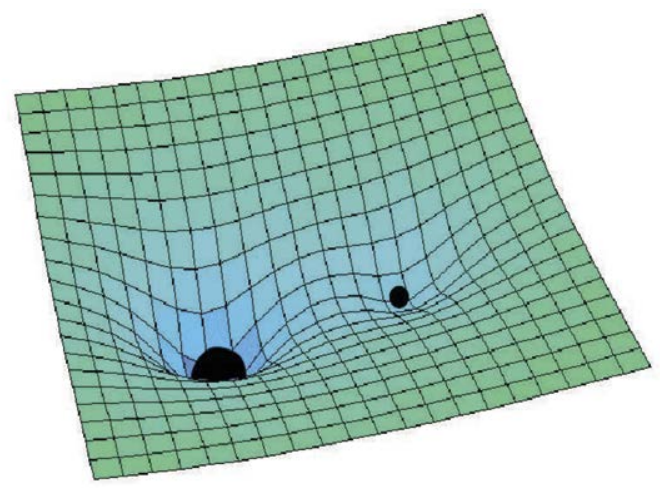

Figura 52 - Rappresentazione dello spazio tempo della relatività generale.

Spazio e tempo, però, hanno una profonda differenza: mentre nello spazio possiamo muoverci in tutte le direzioni, nel tempo ci muoviamo in un solo verso. Non solo: la velocità con la quale possiamo muoverci nello spazio non può superare la velocità della luce. Tuttavia, le equazioni di Einstein ammettono come soluzioni anche i cosiddetti wormholes, sor- 
ta di cunicoli spazio-temporali capaci di unire due regioni separate dello spazio-tempo. Se i wormholes potessero essere aperti e fosse possibile attraversarli, potrebbero essere usati come una 'scorciatoia' tra due punti dell'Universo, che permetterebbe di viaggiare tra di essi più velocemente di quanto impiegherebbe la luce a percorrere la distanza che li divide nello spazio esterno al wormhole (e quindi forse anche per effettuare viaggi nel tempo...).

La comunicazione fra regioni distanti dell'universo avviene mediante onde gravitazionali.

L'onda gravitazionale è una deformazione della curvatura dello spaziotempo che si propaga come un'onda. L'esistenza delle onde gravitazionali fu prevista nel 1916 come conseguenza della relatività generale, ed è stata confermata sperimentalmente nel2015.

L'equazione di campo di Einstein, infatti, ammette soluzioni ondulatorie per la curvatura dello spazio-tempo, così come avviene per il campo elettromagnetico per le equazioni di Maxwell. Le onde gravitazionali possono essere quindi considerate a tutti gli effetti una forma di radiazione gravitazionale. Al passaggio di un'onda gravitazionale, le distanze fra punti nello spazio tridimensionale si contraggono ed espandono ritmicamente: effetto difficile da rilevare, perché anche gli strumenti di misura della distanza subiscono la medesima deformazione.

Fronti d'onda di particolare intensità possono essere generati da fenomeni cosmici in cui enormi masse variano la loro distribuzione in modo repentino, ad esempio nell'esplosione di supernovae o nella collisione di oggetti di massa molte volte maggiore della massa del nostro Sole.

Si conoscono molte possibili sorgenti di onde gravitazionali, tra le quali sistemi binari di stelle, pulsar, esplosioni di supernovae, buchi neri in vibrazione e galassie in formazione; per ognuna di queste fonti il tipo di segnale emesso dovrebbe possedere un 'timbro' caratteristico che identifichi univocamente il tipo di fonte e la causa dell'emissione.

Un sistema stellare binario, formato da due stelle che orbitano intorno a un comune centro di massa, produce onde gravitazionali continue il cui periodo fondamentale è pari a metà del periodo dell'orbita delle due stelle. Anche la nascita di una stella di neutroni dall'esplosione di una supernova dovrebbe essere annunciata dalla trasformazione di circa lo $0,1 \%$ della massa iniziale in onde gravitazionali. Il 'timbro' di queste onde dovrebbe essere di tipo pulsato.

L'interferometro gravitazionale LIGO (USA) e l'equivalente italiano (VIRGO) hanno rivelato onde gravitazionali causate dalla collisione di due buchi neri distanti circa 1 miliardo e 300 milioni di anni luce. Le onde gravitazionali sono state prodotte nell'ultima frazione di secondo del processo di fusione dei due buchi neri, di massa equivalente a circa 29 e 36 masse solari, in un unico buco nero ruotante più massiccio di circa 62 masse solari: le 3 masse solari mancanti al totale della somma equivalgono all'energia emessa durante il processo di fusione dei due buchi neri, sotto forma di onde gravitazionali. 
Lesistenza delle onde gravitazionali fornisce la conferma sperimentale che l'interazione gravitazionale non consiste in un'azione a distanza fra i corpi massicci (come era supposto nel modello newtoniano della gravità), ma implica l'esistenza del campo gravitazionale, anche nel vuoto (ossia anche in mancanza di materia).

\section{La fisica del disordine}

\subsection{Le macchine termiche e l'entropia}

Verso la metà dell'Ottocento, l'indagine sulle macchine termiche portò ad un nuovo capitolo della fisica, la termodinamica, che si occupa delle trasformazioni di calore in lavoro e viceversa. Nella camera di combustione di un motore entrano carbonio e idrogeno (cioè benzina) da una parte e ossigeno (cioè aria) dall'altra (Fig. 53a).

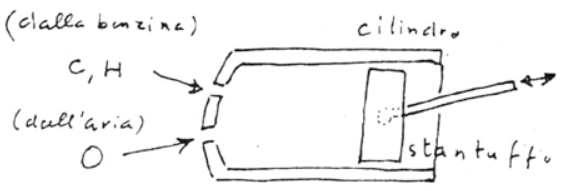

A

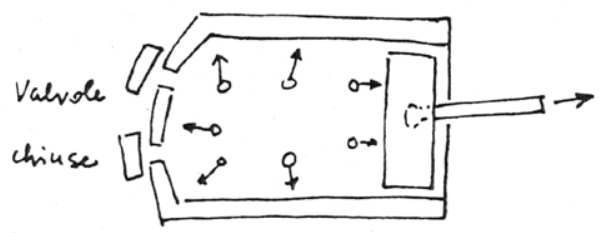

B

Figura 53 - a) Valvole aperte: entrano benzina e aria. b) Nell'esempio in figura, solo 2 molecole spingono nella direzione giusta.

Per fondere le molecole $\left(\mathrm{C}+\mathrm{O}_{2} \rightarrow \mathrm{CO}_{2}, \mathrm{H}_{2}+\mathrm{O} \rightarrow \mathrm{H}_{2} \mathrm{O}\right)$, cioè per fare la combustione, occorre vincere la barriera di potenziale tra le diverse molecole in modo che si passi da $A$ (C, H e O separati) a $B$ (C, H e O fusi) (Fig. 54).

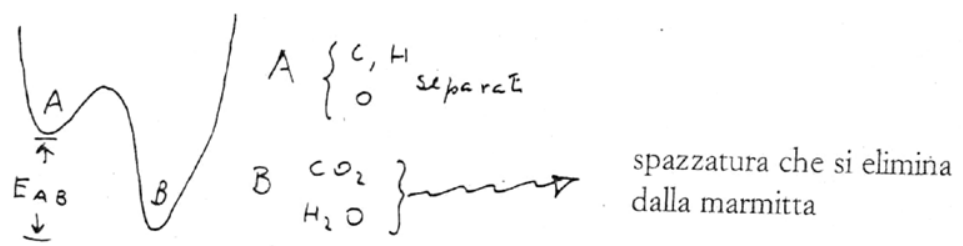

Figura 54 - Diagramma energetico della combustione, o ossidazione. 
Lenergia $E_{A B}$ ottenuta passando dalla buca di potenziale $A$ a $B$ va in energia cinetica (cioè in velocità) dalla molecola risultante $\left(\mathrm{CO}_{2}, \mathrm{H}_{2} \mathrm{O}\right)$ e questa spinge il cilindro. Non tutta l'energia prodotta però viene utilizzata; gran parte viene eliminata dalla marmitta sotto forma di calore (gas di scarico) inutilizzabile. Come mai? Ricordiamo che:

$$
\text { energia cinetica }+ \text { energia potenziale }=\text { costante }
$$

Nella camera di combustione sono state immesse delle molecole che si sono poi fuse, mediante la temperatura che ha fornito l'energia di attivazione per superare la barriera, in una molecola di anidride carbonica $\left(\mathrm{CO}_{2}\right)$ o acqua $\left(\mathrm{H}_{2} \mathrm{O}\right)$. Questa molecola è diventata più veloce di quelle di partenza e perciò la sua energia cinetica può fornire una grossa spinta. Il lavoro utile prodotto dalla folla di molecole non è però uguale alla somma di tutte le energie cinetiche. Le molecole non vanno tutte nella direzione utile, cioè verso lo stantuffo, ma si disperdono in tutte le direzioni riscaldando le pareti del cilindro (Fig. 53 b).

Per questo è necessario raffreddare le pareti del cilindro; inoltre il gas di scarico viene eliminato quando è ancora caldo, cioè ha ancora energia. Nelle normali macchine termiche, si utilizza circa 1 molecola su cinquesei per fornire il lavoro che fa muovere il pistone. Si chiama diavoletto di Maxwell un ipotetico essere in grado di orientare la velocità delle molecole in modo da sfruttare tutta l'energia cinetica sullo stantuffo.

Dall'osservazione del singolo processo, non si capisce lo sparpagliamento delle molecole. La fisica dell'ordine non fornisce spiegazioni sufficienti a capire le folle. Nel cilindro, tutte le molecole hanno la loro energia cinetica 'legittima'. Ogni molecola considerata singolarmente si comporta in maniera onesta, tutte le molecole però prese insieme - cioè considerate come folla - hanno un comportamento disordinato perché vanno in ogni direzione in modo tale che ne servono solo alcune, le altre danno fastidio, e alla fine tutte le molecole sono espulse dallo scarico, lasciando un po' di energia residua da smaltire come calore.

Se spostiamo 1'attenzione dalle molecole al rendimento della macchina, si ha allora che:

rendimento $=($ en. utile $) /($ en. totale $)=($ en. utile $) /($ en. utile + en. espulsa $)$

La 'colpa' del rendimento limitato non è a livello della singola molecola che dà il suo $\mathrm{eV}$ : è la folla che combina guai perché le molecole non sono concordi. Si introduce così il concetto di disordine, cioè il fatto che non tutte le molecole si orientano nella direzione utile. Si chiama entropia il grado di disordine che caratterizza un sistema termodinamico.

Quando un sistema è all'equilibrio termico, cioè ha una temperatura uniforme, esso si trova nella situazione di massimo disordine.

Una nuova legge fisica (II principio della termodinamica) dice che l'entropia tende ad aumentare, raggiungendo il massimo all'equilibrio. Si tratta 
di una qualità in un universo di quantità: la freccia del tempo, una natura che evolve solo in una direzione e non può tornare indietro. Infatti se raccogliamo delle molecole di un gas in un contenitore chiuso, a contatto con un contenitore vuoto, e apriamo la parete fra i due contenitori, si verifica una condizione di massimo disordine: le molecole si sparpagliano ovunque e non è poi possibile farle tornare tutte nel contenitore iniziale (Fig. 55).

Similmente, supponiamo di gettare una goccia d'inchiostro in un bicchiere d'acqua. Con il passare del tempo, la goccia si spande e, dopo un po', l'acqua del bicchiere è diventata uniformemente scura. Non è mai successo che, al passare del tempo, una goccia d'inchiostro si riconcentri e l'acqua torni limpida.

Se riuscissimo ad orientare le velocità delle molecole in una stessa direzione, otterremmo il massimo rendimento. Alla fine dell'Ottocento quindi gli studi sull'energia erano arrivati alla conclusione che l'energia totale è costante ma se ne utilizza solo una parte. Per esempio, l'acqua tiepida a $40^{\circ} \mathrm{C}$ contenuta in una vasca da bagno possiede molta energia, ma non posso utilizzarla per fare il caffè. Per avere un buon caffè occorre poca acqua ma a $100^{\circ} \mathrm{C}$ e la quantità di energia per scaldare poca acqua fino a $100^{\circ} \mathrm{C}$ è minore di quella necessaria per una vasca piena d'acqua tiepida.
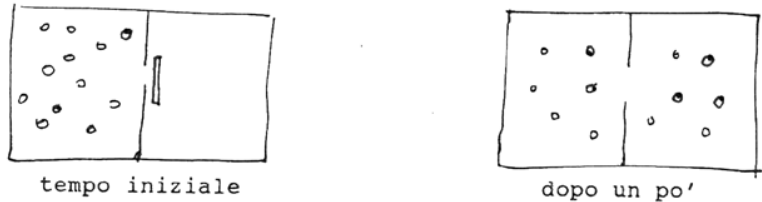

Figura 55 - Perché l'entropia aumenta all'aumentare del tempo.

Quanto detto per le molecole nella camera di combustione vale anche per le singole onde emesse dagli atomi di una lampadina. Le varie onde sono disordinate in fase, una rispetto all'altra, perciò in genere non avremo un'onda globale, ma la somma di tante ondette con fasi a caso (cosiddetta emissione spontanea). Il risultato sarà che avremo solo energia luminosa, ma non osserveremo vistosi effetti ondulatori. Se ora invece ordiniamo le emissioni atomiche, come oggi riusciamo a fare con il laser, allora avremo un'onda con una singola fase.

\subsection{Probabilità}

La parola probabilità viene usata per indicare numerosi concetti tra loro diversi. Conviene pertanto procedere ad un esame di questi concetti per renderci conto di quali siano i limiti dell'uso di ciascuno di essi.

Il concetto elementare di probabilità è nato quando si è cominciato a riflettere su alcuni interrogativi posti dai giochi d'azzardo. Laplace (1812) definì la probabilità come rapporto tra il numero dei casi favorevoli e il 
numero dei casi possibili. La definizione è idonea a teorizzare i giochi d'azzardo onestamente giocati, ma del tutto impotente di fronte a situazioni più complicate. Come servirsene, ad esempio, nel caso di una partita ai dadi con i dadi truccati?

Il concetto elementare di probabilità si basa sull'ipotesi implicita che il sistema che dà luogo agli eventi alternativi soddisfi ad un ben determinato principio di simmetria. La difficoltà fondamentale sta nel fatto che è necessario, in qualche modo, affermare che gli eventi alternativi possibili debbono essere tutti equiprobabili, ma senza coinvolgere il concetto di probabilità, che è appunto ancora da definire.

Per superare tale difficoltà, Bernoulli aveva introdotto il cosiddetto principio di ragione non sufficiente (in seguito chiamato anche principio di indifferenza), imparentato con il principio di ragione sufficiente di Leibniz: «In mancanza di ragioni che distinguano, in qualche modo privilegiandoli, alcuni, tra più eventi alternativi, tali eventi debbono tutti considerarsi equiprobabili» (Ars Conjectandi, 1713). Oggi, tale principio verrebbe espresso in modo diverso: «Nulla nella fisica del processo che dà luogo agli eventi alternativi deve cambiare, se ciò che determina uno qualsiasi di tali eventi viene scambiato con ciò che determina un altro qualsiasi di essi». Si tratta appunto, come si vede, di un principio di simmetria.

Questo principio, se valido, giustifica il fatto che si attribuisca a tutti gli eventi possibili la stessa probabilità a priori, per cui basta contare tali eventi per ottenere la probabilità di ciascuno. Così, la probabilità di uno qualunque dei sei esiti diversi di un dado non truccato risulta essere 1/6 e questi valori delle probabilità rendono conto in modo soddisfacente dell'andamento delle vincite e delle perdite in partite giocate onestamente. Ma se i dadi sono truccati, vincite e perdite si discostano sistematicamente da quanto ci si aspetterebbe sulla base di quelle probabilità.

Ciò porta all'idea che, in una situazione del genere, i singoli casi possibili non siano più equiprobabili. A rigore, quest'ultima frase è priva di senso, se non si dispone del concetto elementare di probabilità. Diremo allora che il desiderio di poter fare previsioni anche con dadi truccati pone l'esigenza di un nuovo concetto di probabilità, in cui la probabilità possa anche risultare diversa per diversi casi a priori possibili. Per far fronte a queste esigenze sono stati introdotti altri tre concetti di probabilità: 1) il concetto frequentistico o statistico, 2) il concetto logicistico, 3) il concetto di probabilità soggettiva.

\section{1) Concetto Frequentistico}

Secondo il concetto frequentistico, non si può parlare (al contrario di quanto si fa nel linguaggio corrente) di probabilità a proposito di un singolo evento, ma solo a proposito di una classe, o collezione di eventi dello stesso tipo, ciascuno dei quali si presenti con una tra due o più modalità alternative diverse. La probabilità di ciascuna modalità viene ancora riportata al rapporto fra il numero dei casi favorevoli e il numero dei casi possibili, ma questi non vanno contati prendendo in considerazione a priori le 
alternative disponibili, bensì a posteriori, esaminando l'insieme di eventi una volta che questi si siano prodotti. Così nel lancio di un dado la probabilità del 4 non è quella elementare; invece si deve eseguire una serie di $N$ lanci (con $N$ molto grande), contare il numero dei 4 ottenuti e dividerlo per $N$ : in tal modo si ottiene la frequenza dei 4 nella serie di lanci. La probabilità del 4 è il 'limite' della relativa frequenza al tendere ad infinito del numero N. Qui la parola limite non è usata nel senso rigoroso dell'analisi matematica e ciò costituisce la difficoltà di questo concetto. A parte ciò, esso è evidentemente utilizzabile anche per dadi truccati.

Il concetto statistico è stato introdotto da John Venn nell'Ottocento. Il suo aspetto più importante è che presenta la probabilità come un dato oggettivo, ricavabile dall'esperimento.

\section{2) Concetto logicistico}

Il concetto logicistico concepisce la probabilità di un evento come una relazione logica fra l'evento e le conoscenze di cui si dispone al riguardo. Con maggior precisione, questa probabilità è una relazione fra proposizioni. Così, non ci si deve chiedere «qual è la probabilità che domani piova?» ma «qual è la probabilità che la proposizione "domani pioverà" sia vera?». La probabilità dipende quindi dalle conoscenze di cui disponiamo, e siccome anche queste sono espresse mediante proposizioni, la probabilità è una relazione fra proposizioni, cioè una 'relazione logica'. Come tale è anche una relazione oggettiva, ma ad un livello di oggettività diverso dalla probabilità statistica: non già a livello degli eventi, ma a quello del nostro discorso sugli eventi.

Il concetto logicistico di probabilità è stato introdotto da George Boole nell'Ottocento e ripresentato da Ludwig Wittgenstein nel Tractatus (1921) e sviluppato criticamente da John Maynard Keynes (1921).

\section{3) Probabilità sogget tiva}

La probabilità soggettiva corrisponde ad un apprezzamento quantitativo di una situazione meramente soggettiva, cioè è l'ammontare della fiducia che il soggetto pensante ha nel verificarsi di un certo evento. È pertanto un valore soggettivo di regola variabile da individuo ad individuo. Questo concetto risale all'Ottocento ed è stato ripreso nel Novecento da Bruno de Finetti. Secondo de Finetti, non esistono eventi ripetibili e non ha quindi senso il concetto statistico di probabilità. La probabilità, come grado di credenza nel verificarsi di un certo evento, si valuta quantitativamente in base alla massima posta che il soggetto è disposto a rischiare in una scommessa, posta espressa come frazione del premio che riscuoterà se l'evento si verifica. Se l'evento non si verifica il soggetto perde la posta e non vince niente. Questo concetto è pertanto di pertinenza esclusiva della psicologia. Anche una frequenza sperimentale (osservata ad esempio in una serie di lanci ripetuti di un dado) non va confusa secondo questa concezione con una probabilità, poiché di fatto non esistono eventi ripetibili e il soggetto può benissimo non dare alcun peso a tale frequenza come indicazione del 
grado di simmetria e bilanciamento del dado. La probabilità così intesa non ha quindi alcun carattere di oggettività.

A questo punto siamo in grado di rispondere alla domanda: fra i vari concetti di probabilità quale va utilizzato nelle scienze sperimentali?

Il calcolo delle probabilità è oggi un ramo della matematica che si sviluppa per via deduttiva da un sistema di assiomi; come tale, esso è completamente indipendente dal significato intuitivo dei termini che compaiono negli assiomi. Il calcolo della probabilità è dunque uno e uno solo, ma i termini che compaiono in esso debbono prima di tutto essere interpretati, cioè acquistare un significato nel campo di indagine in questione. È in questo campo, dunque, che si deve definire un conveniente concetto di probabilità e dimostrare poi che tale concetto soddisfa gli assiomi del calcolo.

Se ne conclude che i tre diversi concetti di probabilità non corrispondono a tre teorie, ma a tre diverse interpretazioni del calcolo delle probabilità. Il problema è ora vedere quale serve nelle scienze sperimentali. Possiamo senz'altro escludere le interpretazioni soggettiva e logicistica. Il campo della prima è privo di quei caratteri di obbiettività che si richiedono a ciò che è oggetto di scienza. Né, d'altra parte, una relazione (logica) tra proposizioni può dirci da sola alcunché degli aspetti del mondo: in modo analogo, la deducibilità di un teorema in un sistema deduttivo non ci dice nulla sul fatto che il relativo corpo di assiomi possa o meno essere usato per descrivere certi aspetti della realtà.

Ci resta così per le scienze sperimentali solo il concetto statistico. L'interpretazione statistica del calcolo delle probabilità implica tuttavia condizioni molto precise; in particolare, che gli eventi siano ripetibili. Ora, secondo la concezione del mondo della scienza classica, è proprio la regolare ripetitività di certi eventi che rende possibile la formulazione di leggi e quindi la stessa costruzione di una scienza.

La ripetitività di certi eventi in dipendenza dalle loro cause è ciò che ha suggerito che gli eventi siano in qualche modo concatenati. Un primo tipo di concatenazione è quella storica: ciascun evento crea le condizioni necessarie perché l'evento seguente possa verificarsi (un vaso non può cadere da un terrazzo se prima qualcuno non ve lo ha messo). Ma l'idea suggerita dalla costante riproducibilità di certi eventi in certe condizioni è quella della possibilità di una concatenazione più stretta di quella storica: una concatenazione in cui ogni evento non è solo necessario ma anche sufficiente per il successivo. Ė questa propriamente l'idea di 'causa'.

D'altronde l'esempio mostra che esistono eventi (come l'esito del lancio di un dado) che risultano non inseriti in una concatenazione più stretta di quella storica. Un evento del genere è per definizione un evento casuale. Nessuno degli eventi che a priori potrebbero essere presi in considerazione come sue possibili cause risulta collegabile ad esso mediante un legame di necessità.

È propriamente l'esistenza di eventi casuali che rende possibili le applicazioni del calcolo delle probabilità allo studio dei fenomeni naturali. 


\subsection{L'informazione}

È ben noto quale importanza abbia sempre avuto l'informazione nella vita umana. Ma solo in epoca recente, con lo sviluppo della scienza e della tecnologia, ci si è resi conto dell'assoluta priorità dell'informazione rispetto agli altri beni di cui dispone l'uomo. In tutto il secolo XIX e in parte del XX ci si è preoccupati soprattutto delle materie prime, delle risorse agricole, della disponibilità di manodopera. Oggi si tende invece a dare un posto preminente alle informazioni e 'cognizioni', da quelle generali scientifiche, a quelle tecnologiche più spicciole, chiamate know how.

È chiaro che il primo problema da risolvere è quello di 'misurare' l'informazione, cioè quello di caratterizzare ragionevolmente con un numero, con una grandezza fisica, la quantità di informazione.

L'informazione si trasmette mediante messaggi. Daremo a questa parola un'accezione molto larga. Per noi sarà messaggio una parola, un gesto, il suono di una campana, una lettera dell'alfabeto, un punto o una linea dell' alfabeto Morse, una pagina stampata, un libro e così via: chi trasmette un messaggio potrà scegliere da un insieme costituito da un certo numero $n$ di messaggi diversi. Per chi trasmette 'punto' o 'linea' sarà $n=2$, per chi indica di andare a 'destra' o 'a sinistra' o 'diritto' sarà $n=3$, per chi batte un carattere a macchina sarà $n=32$ (ponendo che la macchina da scrivere abbia 32 tasti) e così via.

È chiaro che un determinato messaggio porterà tanta più informazione quanto maggiore è il numero $n$ dei messaggi tra i quali può essere scelto. Per esempio, un semplice 'sì o 'no' $(n=2)$ porterà un' informazione molto modesta; invece un discorso di dieci parole può portare un' informazione enorme, perché enorme è il numero $n$ dei messaggi differenti che si possono formare con dieci parole.

Dunque, sembra opportuno che la misura dell'informazione sia rappresentata da un numero che cresce con $n$. Tale è $n$ stesso o $n^{2}, \mathrm{o} n^{3}, \mathrm{o} \log$ $n$ ecc. Per fare una scelta opportuna dovremo porre qualche altra condizione. La condizione più ragionevole consiste nel richiedere che la trasmissione di due, tre ecc. messaggi consecutivi scelti dallo stesso insieme porti il doppio, il triplo ecc. di informazione rispetto ad un singolo messaggio. Due pagine stampate dovrebbero contenere il doppio d'informazione di una singola pagina, un libro di cento pagine dovrebbe contenere cento volte tale informazione e così via.

Supponiamo di trasmettere una successione di messaggi elementari, scelti fra $n$ diversi. Se ne trasmettiamo due, ciascuna delle $n$ possibilità del primo può combinarsi con una delle $n$ del secondo. Per esempio in una scelta binaria (testa/croce nel lancio della moneta ovvero $+\mathrm{o}-$ ), abbiamo:

un messaggio, 2 possibilità: $(+,-)$ due messaggi, $2^{2}$ possibilità: $(+,+),(+,-),(-,+),(-,-)$ 
In genere perciò avremo $W=n^{2}$ messaggi possibili, mentre l'informazione deve essere doppia. Allora l'informazione è proporzionale all'esponente 2 , cioè al logaritmo di $W$.

Ricordando il grafico della funzione logaritmica, si vede che per $W$ piccolo, l'informazione (che chiameremo I per brevità) cresce rapidamente, mentre per $W$ grosso, $I$ cresce sempre più lentamente. Nell'esempio del lancio della moneta, il messaggio corrispondente a 10 lanci deve avere informazione 10 volte più grande del singolo lancio. Invece di classificare tutte le $2^{10}=1024$ combinazioni di $+\mathrm{e}-$ presi a 10 a $10($ ad es.: $[+-+--$ $-++--]$ ), ci limitiamo a dire che l'informazione è di 10 cifre binarie o, come si dice in gergo, di 10 bit (dove bit è abbreviazione di binary digit).

Stabiliamo un legame fra informazione $I$ e probabilità. Se i $W$ messaggi sono tutti equiprobabili, a ciascuno spetterà una probabilità $p=1 / W$. Ricordiamo dall'aritmetica elementare che una divisione equivale a elevare alla potenza -1 , cioè al posto di $W$ possiamo scrivere $p^{-1}$; pertanto il logaritmo di $W$ è 'meno' il logaritmo di $p$, cioè

$$
I=-\log p
$$

Questo secondo modo di scrivere si può generalizzare al caso in cui i vari messaggi accadano con probabilità differenti. Introduciamo il concetto intuitivo di media (la media fra 3,5 e 7 è $(3+5+7) / 3=5)$, e indichiamo una media con parentesi acute $<>$. Orbene, in generale l'informazione è legata alla espressione media:

$$
I=-<\log p>
$$

Abbiamo dunque collegato informazione a probabilità. Si tratta di un concetto non riportabile immediatamente a un'operazione di misura, come lunghezza o velocità, e il corrispondente apparato di misura non è uno strumento in vendita (non esiste l'informometro). Per una definizione quantitativa, è occorsa tutta una procedura complessa che è stata fonte di discussioni. Tali discussioni nel caso di micro-oggetti (meccanica quantistica) o dei viventi (biologia) non sono ancora finite.

\section{Fisica dei processi percettivi e sincronizzazione di impulsi neuronali}

\subsection{Fisica delle percezioni}

\subsubsection{Fisica chimica dei primi stadi della visione}

Una sorgente luminosa invia i suoi raggi ad un oggetto, su cui vogliamo acquisire informazione. I raggi modificati (trasmessi o riflessi) dall'oggetto sono raccolti dall'occhio ed elaborati dal cervello, che di conseguenza prende delle decisioni. Fra le decisioni, alcune possono ri- 
guardare lo stesso sistema di misura, cioè possono implicare un riaggiustamento per migliorare i dettagli (feedback), talvolta anche mediante l'inserzione di lenti nel cammino sorgente-oggetto (ad esempio il proiettore di diapositive), oppure nel cammino oggetto-occhio (occhiali, microscopi, telescopi).

Tutti i colori visibili corrispondono a fotoni tra $2 \mathrm{eV}$ (rosso) e $4 \mathrm{eV}$ (violetto). La cosa importante è che uno, e un solo fotone per volta, può 'dialogare' con un atomo o una molecola.

Vediamo cosa succede nell'occhio. La cornea e il cristallino trasmettono un'immagine del mondo esterno sullo strato di cellule fotoricettive della retina. Ciascuna cellula assorbe la luce da un punto dell'immagine e genera un segnale elettrico corrispondente alla quantità di luce che viene assorbita.

Negli occhi dei vertebrati ci sono due tipi di cellule ricettive, bastoncelli e coni: entrambi contengono una membrana fotosensibile con molecole di pigmento che assorbe la luce. I bastoncelli (in inglese rods) hanno un pigmento rossastro detto rodopsina. La membrana fotosensibile dei bastoncelli consiste di una pila di dischi che somigliano a delle monete sovrapposte. Una sostanza che diffonde trasporta l'informazione dai dischi, dove la luce è assorbita, alla membrana superficiale, dove si genera il segnale elettrico. Il trasportatore si chiama in gergo cGMP (guanosin-monofosfato ciclico). Vediamo come si genera il segnale elettrico. Fuori dalla cellula la concentrazione di $\mathrm{Na}^{+}$è alta e quella di $\mathrm{K}^{+}$è bassa, mentre all'interno è il contrario. Le differenze di concentrazione sono mantenute da una pompa metabolica. Al buio il flusso di cariche causa una differenza di potenziale di $-40 \mathrm{mV}$. Quando un bastoncello assorbe un fotone si blocca il flusso di sodio: ciò riduce la corrente di buio e aumenta la polarizzazione negativa a $-70 \mathrm{mV}$; l'impulso di $-30 \mathrm{mV}$ ha una durata di meno di un millisecondo.

Come fa l'assorbimento di luce a bloccare gli ioni sodio? Sembra che il cGMP faccia da 'portiere' all'imbocco dei canali ionici. L'apertura di un singolo poro richiede la presenza cooperativa di tre molecole di cGMP: siamo in presenza di un interruttore molecolare che rivela cambiamenti piccolissimi nella concentrazione di cGMP. La catena di eventi molecolari che ne blocca l'azione è stata studiata da George Wald. La rodopsina ha un pigmento con una componente che assorbe la luce, il cis-retinale; quando il retinale nella rodopsina assorbe un fotone, esso cambia configurazione da cis a trans; la molecola diventa enzimaticamente attiva e questa attività permette di spostare il cGMP dall'ingresso dei canali ionici. Un solo fotone sposta centinaia di cGMP e ciò blocca l'entrata di un milione di ioni sodio. In corrispondenza a pochi fotoni al secondo abbiamo una corrente che supera il milione di ioni sodio al secondo: siamo in presenza di un fotomoltiplicatore chimico.

Descriviamo l'interazione tra le cellule fotoricettrici e i fotoni (vedi Fig. 44): normalmente la molecola è a riposo in A; quando arriva un fotone visibile, la molecola acquista l'energia e può poi passare in $\mathrm{B}$. Da $\mathrm{B}$ ritorna 
in A e la differenza di energia è restituita sotto forma di segnale elettrico che viaggia nel nervo ottico. Abbiamo cioè visto un fotone!

Se però il fotone è IR, è troppo piccolo per permettere il passaggio A-B e non registriamo niente. Se invece il fotone è UV, la molecola si può dissociare. Per fortuna le parti esterne dell'occhio assorbono i fotoni UV e impediscono che la retina sia distrutta.

\subsubsection{Gli stadi del processo visivo}

Il processo visivo inizia dunque con la trasformazione dei fotoni in segnali elettrici. Questi vengono trasmessi attraverso un complesso sistema di giunzioni nervose, in cui segnali provenienti da diversi fotoricettori sono sommati e confrontati. Ciò permette di ottenere informazioni sulla forma, il movimento e il colore del mondo circostante. Cerchiamo di vedere in maggior dettaglio come ciò avvenga.

La retina è composta da 125 milioni di fotoricettori. Le loro fibre nervose, o assoni, si suddividono in un certo numero di rami che portano a un secondo stadio di cellule nervose. Queste a loro volta sono connesse con un terzo stadio di cellule chiamate cellule gangliari. Gli assoni di queste cellule gangliari si riuniscono a formare il nervo ottico. Da ciascun occhio esce circa un milione di fibre del nervo ottico. Alcune fibre restano nello stesso lato del cervello dell'occhio da cui escono, mentre altre vanno al lato opposto. Tutte queste fibre terminano su due gruppi di cellule, $\mathrm{i}$ corpi genicolati laterali, che rappresentano come delle stazioni intermedie tra la partenza (retina) e l'arrivo (corteccia visiva primaria) (Fig. 56). Da qui, dopo essere passata da uno strato all'altro attraverso diversi insiemi di cellule connesse sinapticamente, l'informazione è inviata a una serie di aree visive superiori, situate nelle vicinanze; ognuna di queste distribuisce l'informazione su molte altre aree.

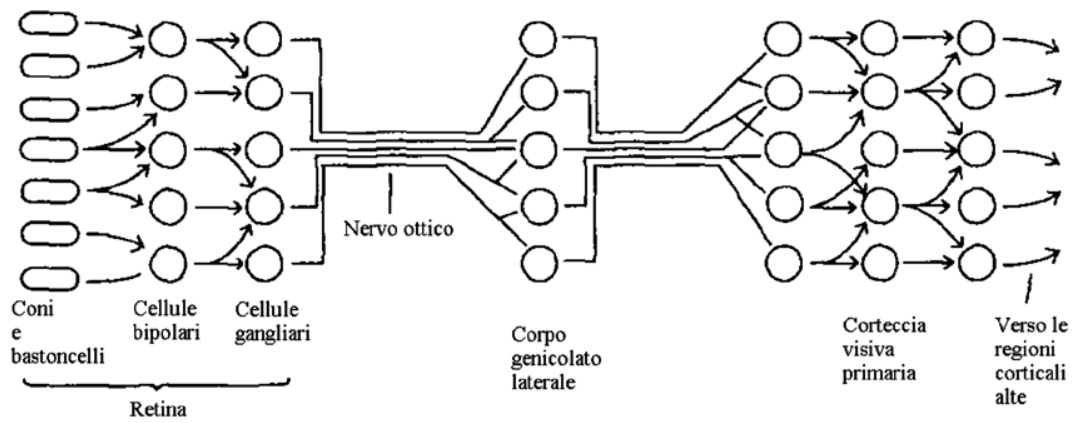

Figura 56 - Percorso del segnale visivo dalla retina alle aree corticali più alte. A ciascuno stadio una cellula riceve un segnale di ingresso da molte cellule, e a sua volta invia il proprio segnale a molte cellule. Sono mostrati pochi neuroni, mentre il processo reale coinvolge milioni di neuroni. 
Possiamo dire che l'assone di ogni cellula è come una linea di trasmissione su cui l'informazione si propaga sotto forma di impulsi elettrici. Al 'bottone' di connessione fra una cellula e l'altra (sinapsi) l'informazione è veicolata da neurotrasmettitori. Ogni cellula si comporta come un circuito a soglia, che si 'accende' quando la somma algebrica dei segnali di ingresso (eccitatori meno inibitori) supera un certo valore.

Per comprendere meglio il passaggio di tali segnali, immaginiamo di avere un animale di fronte a uno schermo e di posizionare un microelettrodo all'interno di una fibra del nervo ottico. Variando i segnali luminosi sullo schermo e osservando la risposta del micro-elettrodo possiamo capire quale segnale debba arrivare sulla retina per influenzare la fibra selezionata. Ciò che si osserva è che l'area della retina che influenza una fibra del nervo ottico ha tipicamente dimensioni pari a circa un millimetro di diametro. Questa regione è chia mata campo ricettivo della cellula. Il campo ricettivo di una singola cellula gangliare ha, sulla retina, una dimensione di circa $1 \mathrm{~mm}$ di diametro, in modo da sottendere un arco di 3,5 gradi, che corrisponde ad un oggetto di circa $1 \mathrm{~cm}$ di diametro alla distanza di 2 metri.

Se confiniamo la luce in modo che raggiunga solo una zona centrale molto piccola del campo ricettivo, possiamo forzare le cellule gangliari a produrre fino a 50 impulsi durante il primo decimo di secondo della stimolazione (Fig. 57, $4^{\circ}$ riga). In seguito la scarica si stabilizza con un ritmo di 100 impulsi al secondo. Se invece illuminiamo l'intero campo ricettivo la cellula risponderà con molto meno impulsi (Fig. 57, $2^{\circ}$ riga). Infatti la cellula fa un confronto tra la luce che colpisce la piccola zona centrale del campo ricettivo e quella che colpisce il bordo esterno. L'illuminazione della zona centrale può ad esempio eccitare la cellula, mentre quella del bordo può inibirla. Queste cellule gangliari vengono dette avere una zona centrale on e una periferia off. Esistono anche cellule gangliari con una zona centrale inibitoria e una periferia eccitatoria. Le prime forniscono una risposta massima in caso di stimolo luminoso su sfondo scuro, mentre le seconde reagiscono a macchie scure su uno sfondo luminoso.

Anche le cellule del corpo genicolato laterale hanno un comportamento che può essere descritto in maniera simile. Considerando le cellule della corteccia visiva primaria, incontriamo diversi stadi nell'elaborazione dell'informazione. Le cellule del primo stadio hanno un comportamento 'centro-periferia' simile alle cellule genicolate. Anche nel secondo stadio vi sono cellule con una zona inibitoria e una eccitatoria, ma le geometrie di questi campi ricettivi sono diverse da quelle prima considerate. Tali campi sono realizzati per vedere una linea luminosa su sfondo scuro, una linea scura su sfondo luminoso, o un confine tra zona chiara e scura. Quello che abbiamo presentato è solo un esempio. Per ulteriori dettagli, si rimanda al libro di David H. Hubel (Hubel, 1989) che, insieme a Torsten Wiesel, ha avuto un ruolo pionieristico nell'esplorazione di questi processi. 

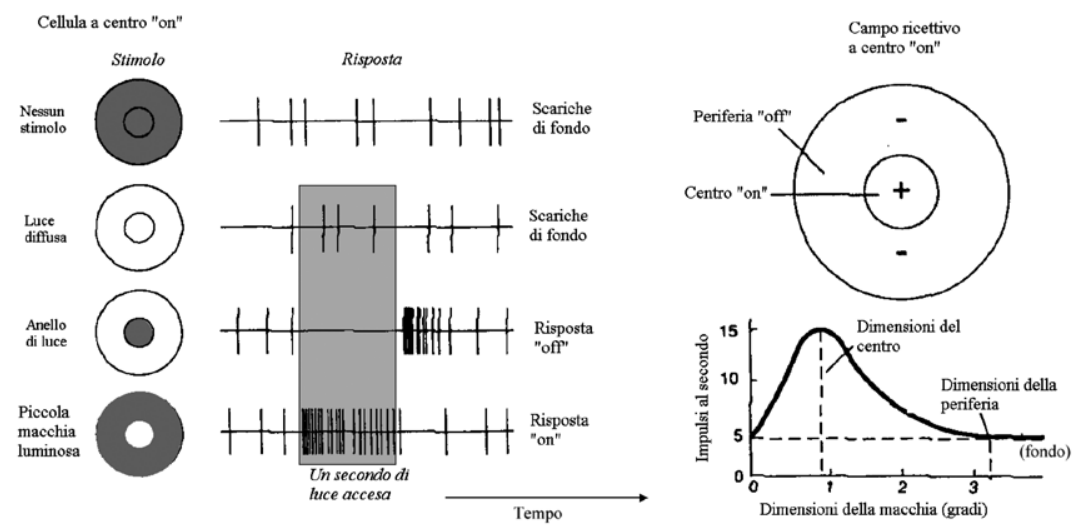

Figura 57 - Risposte di cellule del corpo genicolato aventi il campo ricettivo di tipo centro-periferia. Nel diagramma temporale ciascuna linea verticale rappresenta un singolo impulso registrato nella cellula. In assenza di stimoli luminosi o con luce che copre l'intero campo ricettivo, la cellula scarica casualmente. Con una piccola macchia luminosa al centro, la cellula scarica rapidamente. Invece un anello di luce sulla periferia inibisce la cellula. Il grafico a destra mostra la risposta di una cellula, di tipo centro-on, in funzione della dimensione della macchia. La risposta massima si ottiene quando la macchia luminosa (1 grado) copre solo la zona centrale on. Quando la macchia invade la zona inibitoria, il numero di impulsi diminuisce.

\subsection{Feature binding e codice temporale}

\subsubsection{Il feature binding (integrazione neuronale)}

L'integrazione neuronale consiste nell'instaurarsi di un legame fra neuroni anche distanti i cui campi ricettivi hanno estratto dettagli diversi della stessa figura. Tale legame (feature binding) rappresenta uno stato collettivo caratterizzato da tutti quei neuroni, anche distanti, i cui treni di impulsi si sincronizzino. Studi psicofisici hanno mostrato che l'analisi di scene visuali implica almeno due fasi: nella prima, le strutture elementari degli oggetti, il colore, il movimento e l'orientazione dei contorni, sono rivelate in maniera locale e parallela; successivamente, questi componenti sono connessi in unità organizzate per fornire la base di una rappresentazione coerente degli oggetti. Come già mostrato dagli studi degli psicologi della Gestalt, questo processo segue certi criteri che includono per esempio la prossimità o la somiglianza delle strutture.

Ovviamente il problema del legame, come descritto nel campo della psicofisica, ha il suo corrispettivo ad un livello neurobiologico. Un meccanismo per l'integrazione è necessario per molte ragioni. Per prima cosa, i neuroni nei centri visivi hanno campi ricettivi ristretti che, nei primi stadi dell'e- 
laborazione, sono piccoli rispetto alle dimensioni tipiche degli oggetti. In secondo luogo, il meccanismo deve agire su distanze considerevoli per permettere il legame tra differenti aree visive. È accertato che differenti classi di strutture di oggetti siano elaborate in distinte aree corticali servendosi di mappe di struttura. Nella scimmia sono state individuate più di trenta aree visive, e nel gatto venti, che differiscono nella proprietà di risposta dei loro neuroni. Quindi, l'attività neuronale di diverse aree deve essere integrata per stabilire una rappresentazione degli oggetti complessi. Inoltre, un meccanismo di legame è necessario, dal momento che sono spesso presenti nel campo visivo molti oggetti che attivano neuroni in un grande numero di aree corticali. Allora si deve poter selezionare la struttura corrispondente a un determinato oggetto, separando le risposte relative ad esso da quelle dei neuroni attivati da altri oggetti per evitare un'illusoria identità di strutture.

È stato suggerito che il problema del legame potesse essere risolto da un meccanismo di integrazione temporale (Von der Malsburg) consistente nella connessione in insiemi coerenti di neuroni corticali grazie alle sincronizzazione delle loro scariche. La sincronizzazione tra scariche neuronali potrebbe fornire un modo di etichettare le risposte come funzionalmente correlate e di selezionarle per ulteriori meccanismi associati, dal momento che la sincronizzazione può essere facilmente rivelata con la coincidenza di neuroni in altre aree del cervello (Singer e Gray, 1995): si veda al riguardo la figura 33. Lassenza di sincronizzazione tra differenti insiemi potrebbe essere utilizzata per isolare lo sfondo e ottenere una segmentazione delle scene visive.

Questo meccanismo di legame temporale evita i tranelli di precedenti modelli. Era stato suggerito (Barlow) che oggetti complessi potessero essere rappresentati dall'attività di un numero molto piccolo di neuroni o addirittura da uno solo. Il difetto di questa teoria del neurone singolo o neurone della nonna è che ogni nuovo oggetto richiederebbe l'arruolamento' di nuove cellule dedicate nella corteccia visiva. Quindi il numero di neuroni richiesto per un'adeguata rappresentazione di un ambiente visivo crescerebbe oltre ogni plausibile stima. Il legame temporale evita questa limitazione dal momento che gli stessi neuroni possono essere ricombinati in nuove strutture rappresentazionali semplicemente cambiando la loro relazione temporale.

Lassunto del feature binding è che la sincronizzazione stabilisca legami che non sarebbero possibili se le uniche dimensioni di codifica fossero la frequenza degli impulsi e i livelli di attivazione.

Infine, l'influsso top-down, dovuto a memorie che stabiliscono aspettazioni e che riaggiustano le soglie di scarica dei vari neuroni in modo da sincronizzarli (come visto in figura 34, presentando il caso omoclinico), gioca un ruolo importante per la segmentazione di una scena e l'isolamento dello sfondo.

\subsubsection{Il feature binding negli altri sistemi funzionali}

Fenomeni simili sono riscontrabili in altri sistemi funzionali. La sincronizzazione, ad esempio, è nota come importante nell'apparato olfat- 
tivo (W. Freeman). Nella corteccia uditiva, si sono osservate oscillazioni sincronizzate. Negli umani sarebbe traumatico inserire micro-elettrodi che sentano l'attività del singolo neurone (come si fa con animali da laboratorio);questi fenomeni sono stati osservati grazie a tecniche non invasive di EEG e MEG (elettro e magneto-encefalogramma).

Vi sono evidenze sperimentali che suggeriscono che la sincronizzazione possa giocare un ruolo importante per l'integrazione neurale sensoriomotoria. Nelle scimmie è stata registrata la sincronizzazione tra le aree corticali sensoria e motoria. La sincronizzazione delle risposte neuronali non accade solo all'interno del sistema visivo, ma anche tra aree visive e parietali e tra corteccia parietale e motoria. Inoltre, gli esperimenti dimostrano che la coerenza temporale tra le aree registrate cambia a seconda delle situazioni in cui si trova l'animale. Tutti questi fattori suggeriscono che la sincronizzazione assicuri la coordinazione visuo-motoria e metta in rapporto gli aspetti sensoriali e motori del comportamento.

Quest'argomento sarà completato nella Parte $\mathrm{C}$ alle voci Consapevolezza percettiva e NCC e Neuroscienza. 



\title{
PARTE C
}

\section{DIZIONARIO RAGIONATO}

\begin{abstract}
Algoritmo
[dal nome d'origine, al-Khuwärizmì, del matematico arabo MuhammadibnMūsa del IX secolo (così chiamato perché nativo di Khwarizm, nell'Asia Centrale)].
\end{abstract}

1. Termine che indicò nel medioevo i procedimenti di calcolo numerico fondati sopra l'uso delle cifre arabiche. Nell'uso odierno, qualunque schema o procedimento matematico di calcolo descrivibile con un numero finito di regole che conduce al risultato dopo un numero finito di operazioni, cioè di applicazioni delle regole.

2. In informatica, insieme di istruzioni da applicare per eseguire un'elaborazione o risolvere un problema.

3. In logica matematica, qualsiasi procedimento 'effettivo' di computo di una funzione o di decisione di un insieme (o predicato), cioè qualsiasi procedimento che consenta, con un numero finito di passi eseguiti secondo un insieme finito di regole esplicite, di ottenere il valore della funzione per un dato argomento, o di decidere se un dato individuo appartiene all'insieme (o soddisfa il predicato).

4. Nel descrivere i processi cognitivi, chiamiamo algoritmo la probabilità condizionata $P(d \mid h)$ che un dato $d$ consegua da un'ipotesi $h$.

Si può definire dunque come:

sequenza ordinata e finita di passi (operazioni o istruzioni) elementari che conduce a un ben determinato risultato in un tempo finito.

Algoritmo genetico (Holland) (Fig. C1)

L'algoritmo (linea centrale nella figura) che rappresenta la scalata al colle di Bayes (vedi la descrizione in Fig. 5) può essere ottimizzato con una procedura evolutiva detta algoritmo genetico. 


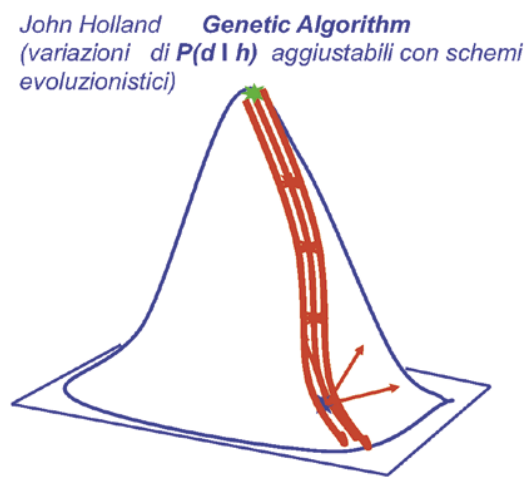

Figura C1 - Lalgoritmo genetico consiste nell'esplorare situazioni vicine a quella di partenza ed effettuare una scelta; l'algoritmo scelto implica una vicinanza a quello di partenza, quindi non si tratta di un 'salto algoritmico' quale presentato in Fig. 15, ma di piccole perturbazioni che mantengono la struttura dell'algoritmo di partenza.

\section{ART (Teoria della Risonanza Adattiva)}

\section{Codifica delle informazioni}

Il cervello esamina gli eventi ambientali concentrandosi sul contesto. Se si guarda un'immagine complessa, come la fotografia di un volto noto, si è in grado di riconoscerla all'istante, mentre è impossibile individuarla guardandola pezzo per pezzo; ciò è dovuto al fatto che il cervello opera sui dati sensoriali in parallelo piuttosto che in sequenza.

Normalmente i segnali visivi provenienti da una certa scena raggiungono i nostri occhi contemporaneamente, così che un'elaborazione parallela comincia nella retina. Per elaborare l'insieme come un intero, esso deve essere ricodificato. Una tale operazione di ricodifica è detta working memory e registra le tracce di memoria a breve termine. Per identificare eventi familiari, il cervello confronta le tracce a breve termine con le categorie che ha immagazzinato. A queste categorie si accede usando le tracce della memoria a lungo termine, che rappresenta le esperienze precedenti che sono state acquisite grazie ad un processo di apprendimento.

\section{Il dilemma stabilità-plasticità}

In qualche modo noi siamo in grado di apprendere rapidamente nuovi fatti, senza essere costretti a dimenticarne altri. Come fa il cervello a tenere memoria in maniera stabile degli avvenimenti precedenti, mantenendo allo stesso modo la plasticità necessaria per imparare nuove cose? 
È il dilemma stabilità-plasticità. Vi sono molti interessanti esempi relativi al sistema visivo e auditivo che mostrano come il cervello potrebbe risolvere tale dilemma. Ciascun esempio può essere spiegato grazie ad un approccio di tipo computazionale detto ART (Teoria della Risonanza Adattiva). Nonostante l'estrema varietà di compiti che il cervello deve compiere, i circuiti neurali che governano molti processi sembrano basarsi su uno stesso insieme di principi computazionali. In parte, la connessione risiede in una caratteristica fondamentale delle funzioni del cervello umano: le nostre percezioni vengono confrontate con precedenti percezioni.

Un aspetto assai interessante emerge da un'immagine chiamata figura di Ehrenstein, che consiste in linee nere tracciate in maniera radiale su un foglio di carta. La mente costruisce un cerchio immaginario all'interno delle linee radiali, il che rende la figura simile al disegno che un bambino potrebbe fare del Sole, un disco luminoso circolare bianco con le linee nere a rappresentare i raggi. Infatti, il disco immaginario appare più luminoso rispetto al resto dello sfondo. Una tale percezione è una proprietà emergente e collettiva di tutte le linee che si genera solo quando esse sono disposte in modo adatto (Fig. C2). Perché vediamo un disco luminoso che non esiste?

Un livello più elevato dell'elaborazione visiva risiede nelle categorie di riconoscimento, che controllano l'aspettativa di quello che potremmo vedere, come una faccia o una lettera. Un aspetto fondamentale del riconoscimento degli oggetti dipende da come noi acquisiamo categorie che includono differenti esempi dello stesso oggetto, come la stessa lettera stampata in differenti dimensioni o forme.
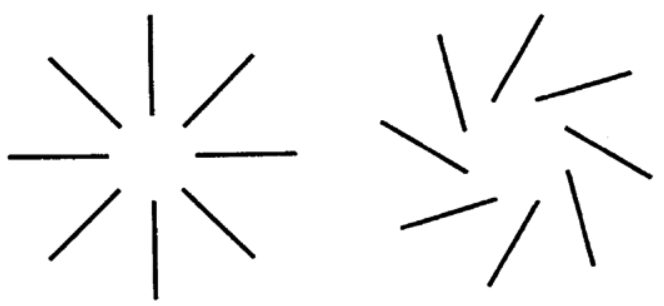

Figura C2 - A sinistra la figura di Ehrenstein. Si percepisce un cerchio illusorio, formato dalle punte interne delle linee, che racchiude unarea apparentemente più luminosa. Una tale percezione dipende dal pattern complessivo, non comparendo in caso di una disposizione diversa (a destra).

\section{Memorie a breve e a lungo termine}

L'ART include due principali tipi di processi di memoria: quella a breve e quella a lungo termine. La memoria a breve termine raccoglie gli sti- 
moli, mentre quella a lungo termine cataloga le informazioni acquisite. Nell'ART l'informazione può passare dalla memoria a breve a quella a lungo termine durante l'apprendimento, e fare il percorso inverso durante il processo di richiamo. Le informazioni vengono codificate nella memoria a breve termine grazie a percorsi di attivazione attraverso una rete di neuroni. La memoria a lungo termine invece codifica grazie a pesi stabiliti adattivamente all'interno dei cammini che uniscono i neuroni nelle varie aree. Questi pesi moltiplicano il segnale durante il suo cammino prima che esso giunga al neurone di arrivo.

Una rete ART opera in accordo a regole specifiche. Così si ha un'accensione di tipo top-down quando, in assenza di ogni segnale di ingresso bottom-up, vengono sensibilizzate cellule che normalmente risponderebbero a una certa classe di stimoli, e altre vengono inibite. Viceversa, se una cellula riceve un segnale bottom-up sufficientemente grande, in assenza di qualunque segnale di ingresso top-down, la cellula genera lo stesso un segnale di uscita, che viene detto attivazione automatica. Durante l'accoppiamento la cellula riceve contemporaneamente segnali di ingresso top-down e bottom-up.

Un processo top-down amplifica selettivamente alcuni aspetti di uno stimolo e ne sopprime altri, aiutando ad accentrare l'attenzione su informazioni che corrispondono alle nostre attese. Questo processo di messa a fuoco ci aiuta a filtrare alcune parti del segnale sensorio, che rischierebbe altrimenti di essere incomprensibile, evitando in questo modo di destabilizzare le categorie memorizzate precedentemente. L'aspettazione di tipo top-down potrebbe risolvere il dilemma stabilità-plasticità, creando un meccanismo di messa a fuoco ed evitando che segnali spuri possano erodere le categorie già memorizzate.

\section{Il meccanismo di feedback e la 'novità'}

D'altro canto, se un'aspettazione top-down è in grado di influenzare uno stimolo bottom-up, nulla impedisce che il segnale bottom-up così modificato possa riattivare le aspettazioni top-down, innescando così un ciclo di feedback. Quando questo ciclo raggiunge l'equilibrio, i segnali bloccano le attivazioni in uno stato risonante. Per spiegare più chiaramente questa risonanza, richiamiamo che gruppi di neuroni devono sincronizzare le loro scariche per dare l'idea del gatto. Ma i campi ricettivi corrispondenti ai vari elementi del gruppo possono avere più o meno luminosità, contrasto, colore, e pertanto dare luogo localmente a sequenze di impulsi più $o$ meno frequenti. Per raggiungere il feature binding, bisogna che dall'alto (memorie pregresse) arrivi un'ipotesi interpretativa che ritocca le soglie di scarica delle cellule, aggiustandone le frequenze di impulsi in modo da creare il legame collettivo. Se la percezione è quella aspettata si crea una risonanza tra top-down e bottom-up per cui la percezione viene confermata: abbiamo visto giusto! È possibile affermare che solo gli stati risonanti del cervello possano condurre alla consapevolezza, e il tempo necessario 
ad arrivare ad un tale risonanza è quello che serve per avere la percezione di un evento (200 ms).

Riassumendo, i segnali top-down rappresentano le aspettazioni acquisite dal cervello di cosa il segnale bottom-up potrebbe rappresentare, aspettazioni basate sull'esperienza passata. Un processo di paragone rafforza e amplifica le caratteristiche del segnale che sono consistenti con le aspettazioni top-down, mentre sopprime le caratteristiche inconsistenti.

Esistono dati neurofisiologici che suggeriscono che il feedback dalla corteccia ai corpi genicolati laterali funzioni proprio come una rete ART, con un circuito di retroazione che esamina le ipotesi fornite da strutture interne all'attività corticale.

Il feedback delle reti ART permette di fornire un modello del riconoscimento visivo degli oggetti. Una rete ART consiste infatti in un sottosistema attenzionale che è in grado di creare categorie e aspettazioni in risposta a segnali di ingresso sensoriali. Esiste inoltre un sottosistema orientante che viene attivato da eventi 'nuovi' e che mette in grado il sottosistema attenzionale di categorizzarli in maniera stabile. In altre parole, l'interazione tra i due sottosistemi permette di risolvere il problema stabilità-plasticità in presenza di molti dati sensoriali.

Un segnale di ingresso viene registrato come un pattern di attivazione della memoria a breve termine, che stimola a sua volta la memoria a lungo termine attraverso un processo bottom-up e inibisce il sottosistema orientante. Fintanto che la memoria a breve termine esamina il segnale di ingresso, il sistema orientante resta inattivo, in quanto l'eccitazione provocata dal segnale e l'inibizione provocata dalla memoria a breve termine si equilibrano. Successivamente, la memoria a breve termine attiva una categoria nella memoria a lungo termine che corrisponda il più possibile ai dati sensoriali.

L'attivazione di una categoria può essere interpretata come il 'fare un' $\mathrm{i}$ potesi' riguardo al segnale di ingresso. La categoria prescelta provoca un segnale di uscita che mette in moto il processo top-down. Il segnale topdown quindi funziona da aspettazione, e attivare l'aspettazione può essere interpretato come verificare un'ipotesi.

Se la configurazione nella memoria a breve termine si avvicina molto al segnale originale di ingresso sensoriale, il sottosistema orientante non entra in azione.

Come reagisce invece la rete quando l'aspettazione top-down non coincide con i pattern della memoria a breve termine? In questo caso il sistema attenzionale produce una configurazione modificata della memoria a breve termine, in modo da contenere solo le poche parti che corrispondono all'aspettazione top-down. Questa configurazione rimuove le inibizioni al sottosistema orientante mettendolo in azione. Tale sottosistema invia un segnale che fa ricominciare la ricerca, ripartendo dall'originale segnale sensoriale. Il ciclo continua finché non viene individuata la corretta categoria, o finché non si rende necessario creare una nuova categoria, poiché il sistema si trova di fronte ad una 'novità'. 


\section{Bayes (inferenza di) e Bayes inverso}

L'inferenza di Bayes (1763) consiste nel trovare la probabilità condizionata di $h$, essendo dato $d$ (chiamiamo $h^{*}$ questa ipotesi selezionata da un gruppo $h$ ):

$$
P\left(h^{\star}\right) \equiv P(h \mid d)
$$

cioè la probabilità della ipotesi ottimale compatibile con il dato $d$, sfruttando l'algoritmo $P(d \mid h)$ (Figg. C3 e C4).

\section{BAYES}

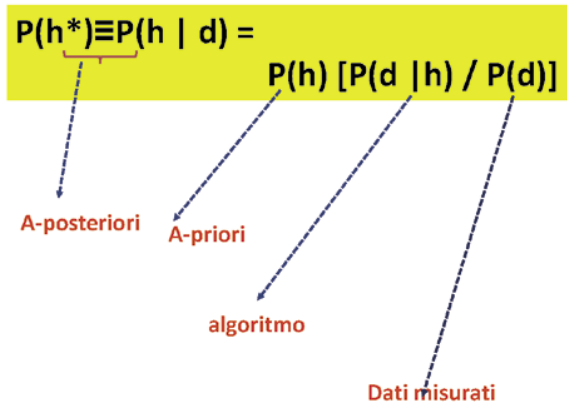

Figura C3 - L’inferenza di Bayes, 1763.

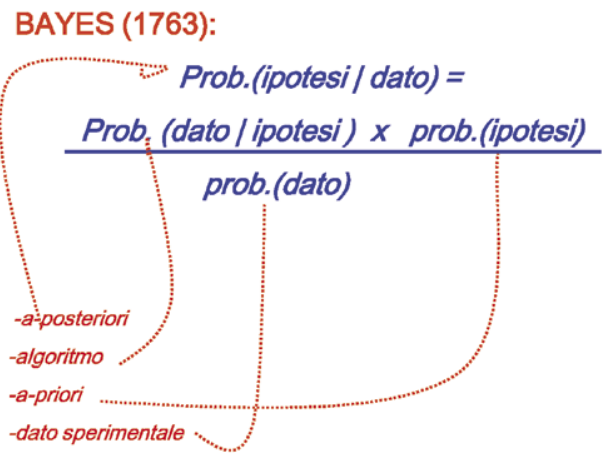

Figura C4 - Esplicitazione con parole della formula della figura precedente.

Confrontiamo la probabilità bayesiana con la certezza newtoniana, cioè:

$$
P(h \mid d)=\frac{P(h) P(d \mid h)}{P(d)} \quad \text { con } d=F(h, t)
$$


Nella dinamica Newtoniana, dato $h$ al tempo iniziale, $d$ emerge con certezza al tempo $t$, pertanto:

$$
P(d \mid h)=1
$$

Se denotiamo con $h$ l'insieme delle ipotesi complementare ad $h$, risulta pertanto:

$$
P(d \mid h)=0
$$

per cui il denominatore di Bayes

$$
\neg h) P(\neg h)
$$

si riduce a

$$
P(d)=P(h)
$$

e ne consegue che

$$
P(h \mid d)=1
$$

cioè in Newton il recupero di $h$ da $d$ è preciso al $100 \%$.

Confrontiamo ora il Bayes diretto con il Bayes inverso, introdotto nella Parte A per il linguaggio (Fig. C5).

\section{Bayes diretto}

L'algoritmo deve essere noto

$P\left(h^{*}\right)=P(h)[P(d \mid h) / P(d)]$

scienza $=$ inferenza di Bayes; algoritmo = teoria $($ Newton Einstein $)$

\section{Bayes inverso}

Se ora le $h^{*}$ sono già date dal flusso delle apprensioni precedenti, allora l'incognita è il 'legame' $P(d \mid h)$, cioè l'algoritmo

$$
P(d \mid h)=P(d) \times P\left(h^{*}\right) / P(h)
$$

Figura C5 - Confronto fra Bayes diretto, in cui si estrae l'ipotesi $h^{\star}$ vincente dato l'algoritmo, e Bayes inverso, in cui si estrae l'algoritmo che correla al meglio due brani $h^{\star}$ e $d$ di un testo linguistico, il precedente richiamato dalla memoria a corto termine e confrontato con il seguente. 
In figura C6 applichiamo Bayes diretto e inverso rispettivamente a 'scienza normale' e 'salto di paradigma' attraverso il riferimento alla distinzione introdotta da T. Kuhn, che così va interpretato: nella scienza normale si opera ricorsivamente per Bayes diretto, avendo come algoritmo una teoria accettata; invece, nel salto di paradigma la lettura linguistica suggerisce il nuovo algoritmo.

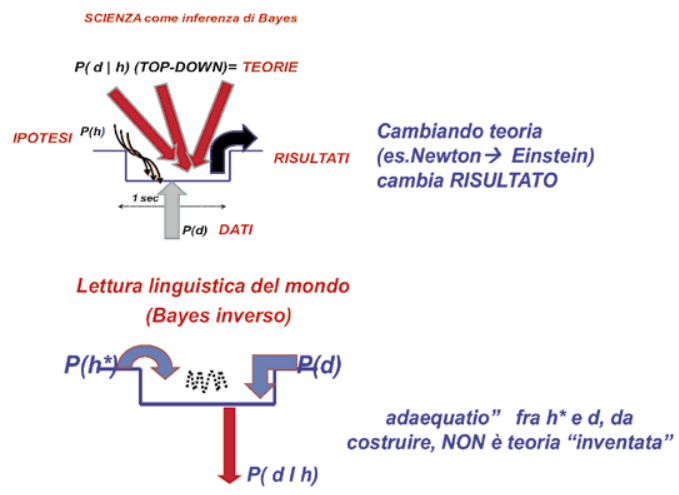

Figura C6 - Confronto fra Bayes diretto e inverso nel programma scientifico: nel primo caso, si ha 'scienza normale', nel secondo si ha 'salto di paradigma'.

\section{Bottom-up (dal basso verso l'alto) e top-down (dall'alto verso il basso)}

Bottom-up denota una fisica che crede di spiegare qualunque oggetto di natura come somma dei suoi componenti. Top-down denota l'evidenza che lo stato di un sistema complesso possa avere comportamenti non riconducibili ai suoi componenti. Esempio: feedback= retroazione (Fig. C7).

Sistemi solo bottom-up (combinazioni di particelle elementari)? No! Anche top-down. Per esempio, il controllo mediante feedback: il comparatore legge la differenza fra obiettivi e stato del sistema e attiva il controllore. In tal modo, variabili astratte come 'obiettivi' acquistano efficacia nel mondo fisico.

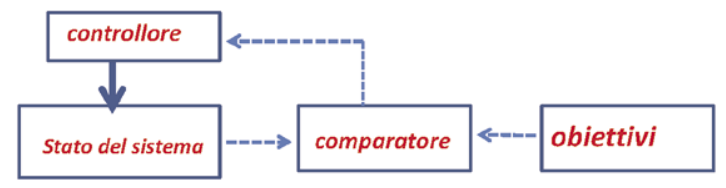

Figura C7 - Ruolo del feedback nel modificare un sistema; il sistema non è pertanto descritto solo dai suoi componenti (bottom up) ma richiede anche la conoscenza delle interazioni col resto del mondo (top-down). 


\section{Caos deterministico, applicazioni e controllo}

Caos deterministico: il modello matematico di un sistema fisico, anche se apparentemente semplice perché consiste di poche equazioni, non garantisce una soluzione che preveda il futuro remoto, in quanto l'informazione si 'consuma' al passare del tempo e va rimpiazzata da nuova informazione.

Il tempo entro cui l'informazione si perde dipende dal sistema: nel caso del sistema solare, esso è at torno al milione di anni (è perciò che il sistema solare ci appare stabile) ma nel caso degli impulsi elettrici (detti spikes) con cui i neuroni del cervello comunicano fra di loro, è solo 2 millesimi di secondo.

\section{Caos geometrico}

Condizioni iniziali vicine portano a risultati molto distanti. Esempio nell'effetto farfalla di E. Lorenz del 1963: se nel raccogliere i dati su cui costruire una previsione meteorologica a Boston trascuriamo il battito d'ali di una farfalla nei Caraibi, possiamo avere fra tre giorni pioggia invece del sereno (Fig. C8).

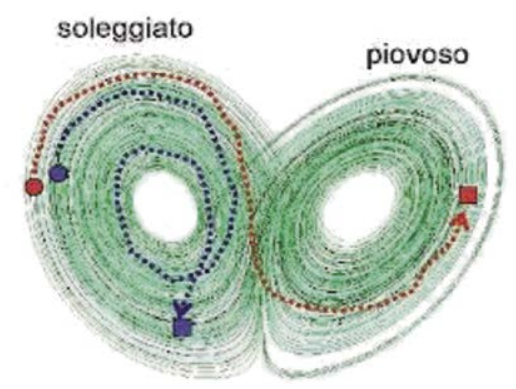

Figura C8 - Caos geometrico-effetto farfalla in modello meteorologico. Il caos geometrico è la dipendenza sensibile da condizione iniziale.

\section{Caos temporale}

Abbiamo segnali di forma invariate (impulsi $=$ spikes) che però si ripresentano a tempi irregolari (vedi la figura 34 nella Parte B e la discussione relativa).

Un vivente reagisce a stimolo sensoriale con risposta motoria. In un animale con cervello, il codice dello stimolo (sequenza di spikes) viene confrontato con il codice di memorie pregresse e ne consegue una 'interpretazione' in base a cui si reagisce, cioè una percezione.

Occorre sincronizzare le spikes di folle di neuroni, perché si adeguino allo stesso codice (la sincronizzazione si raggiunge quando ad esempio una folla batte le mani all'unisono; si veda la figura 33 nella Parte B). Ciò richiede un certo numero di millisecondi (qualche centinaio, fino a 1 secondo), ben oltre i 2 millisecondi entro cui il caos cancella l'informazione di una spike. 
La sincronizzazione implica dunque un 'controllo del caos'; senza questo, saremmo condannati a non 'capire' niente del mondo che ci circonda.

Il controllo del caos è stato discusso nella figura 11 della Parte A.

Caos e dinamica neuronale: il caos omoclinico e la sincronizzazione

\section{Neuroni e laser - Caos omoclinico e sincronizzazione di spikes}

La figura C9 schematizza alcune caratteristiche dinamiche del singolo neurone. Senza entrare nei dettagli del modello (vedi Neuroscienza), rifacciamoci alle considerazioni di opportunità: dal punto di vista della fitness, un neurone caotico risulta vincente ai fini di una codifica rapida e sensibile degli stimoli di ingresso.

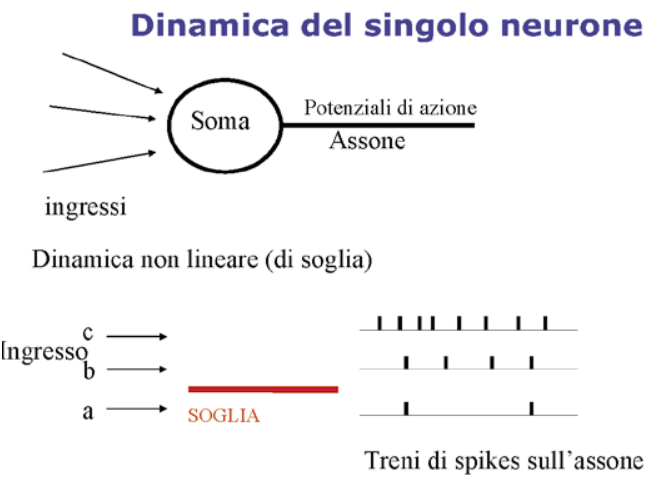

Figura C9 - Schema del singolo neurone. I vari ingressi in arrivo da altri neuroni si sommano nel soma della cellula dove si confrontano con una non linearità, in base a cui generano dei potenziali d'azione che viaggiano sull'assone e arrivano alle sinapsi dove vengono comunicati ad altri neuroni, sotto forma elettrica o chimica (codificando l'attività elettrica dell'assone in flusso di neurotrasmettitori). I tre casi di segnale totale di ingresso con i corrispondenti treni di spikes sembrano suggerire un modello di neurone eccitabile: sotto soglia non cè praticamente niente, sopra soglia si ha una frequenza che cresce con il sopra-soglia.

Presentiamo le condizioni ottimali di un caos individuale da cui emerge una situazione ordinata collettiva, accoppiando folle di individui simili.

Dal 1986 all'Istituto Nazionale di Ottica a Firenze si studia nei laser il caos chiamato omoclinico (HC): vediamone le caratteristiche (Fig. C10).

Studiamo come un singolo HC si sincronizza con un segnale sinusoidale esterno (Fig. C11). A seconda della frequenza della modulazione esterna, si hanno differenti regimi di bloccaggio (locking) della posizione delle spikes alla fase della modulante. Se si sposta gradualmente la frequenza in giù o su rispetto alla frequenza interna, sarà sempre più difficile mantenere il locking (bloccaggio) 1:1. 


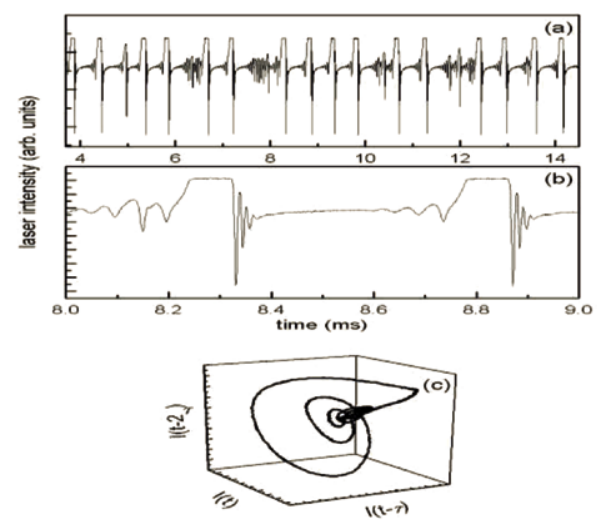

Figura C10 - Segnale sperimentale di intensità di uscita da un laser caotico (caos omoclinico). a) Sequenza di impulsi (spike) quasi identici, separati da intervalli inter-spike. (ISI) erratici, indicazione di caos, come d’altronde si può vedere dai piccoli segnali non ripetitivi nell'ISI. b) Zoom di due impulsi successivi (si veda la magnificazione della scala temporale) per mostrare la ripetibilità dei segnali alti, quelli che chiamiamo spikes. Se introduciamo una soglia possiamo isolare le spikes cancellando i segnali bassi non ripetitivi. c) Riportando su tre assi il valore $I(t)$ dell'intensità al tempo $t$ e i due valori dopo piccoli ritardi $\tau$ e $2 \tau$ (metodo di embedding) si costruisce uno spazio delle fasi tri-dimensionale che ci mostra come l'orbita si chiuda ad ogni giro (più o meno lungo a seconda del valore di ISI). La figura è costruita su molte spikes successive: la parte della spirale percorsa da un sola linea corrisponde agli impulsi alti ripetitivi; la matassa caotica verso il centro corrisponde agli impulsi bassi non ripetitivi.

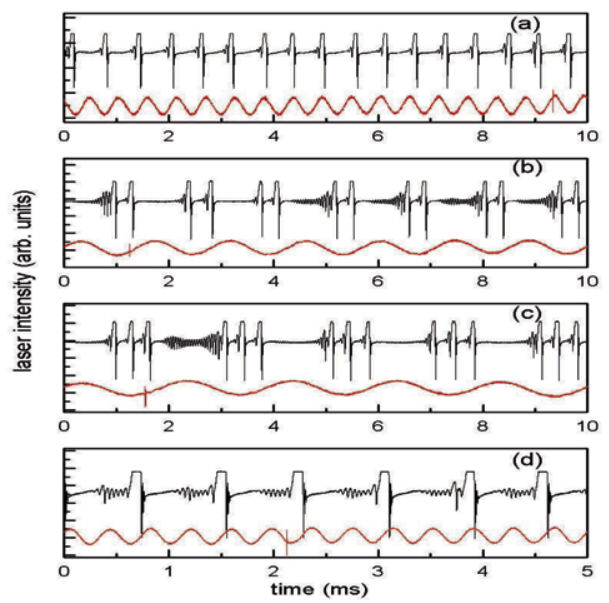

Figura C11 - Sincronizzazione ad una modulante esterna dell'ordine dell'1\% applicata a un parametro di controllo (sinusoidi in basso in a), b), c) e d). a) Se la frequenza è vicina alla frequenza interna dello $\mathrm{HC}$ si ha un bloccaggio (locking) 1:1. b) e c) A più basse frequenze, si hanno locking 1:2 e 1:3. d) A più alta frequenza, si ha locking 2:1. 
Passiamo ora da un singolo $\mathrm{HC}$ con modulazione esterna a una rete di 40 siti HC accoppiati fra prossimi vicini (cioè il sito $i$ invia segnali a, e ne riceve da, ) (Fig. C12).
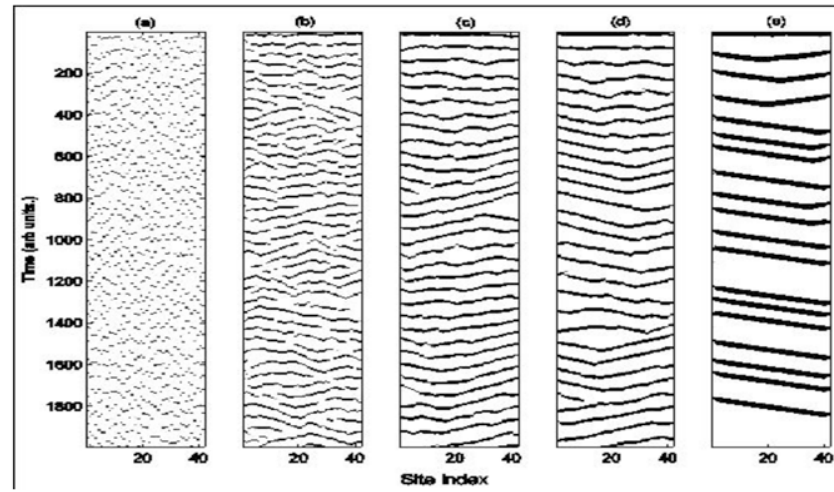

Figura C12 - Diagrammi sito(orizzontale)-tempo (verticale) dei treni di spikes HC, ogni spike essendo rappresentata da un punto. Grado di accoppiamento $\varepsilon=$ a) 0.0 ; b) 0.05 ; c) 0.1 ;) 0.12 ; e) 0.25 . Per alto accoppiamento le spike sono sincronizzate su tutti i siti a meno di un ritardo fra un sito e il successivo; in assenza di accoppiamento $(\varepsilon=0)$ ogni HC va per conto proprio.

Dunque i 40 siti si sincronizzano per un certo grado di accoppiamento mutuo. Questo esempio si riferiva alla sincronizzazione spontanea in assenza di uno stimolo esterno e, come si vede con un taglio verticale, ogni singolo sito si mantiene HC nel tempo. Analogamente possiamo studiare la sincronizzazione di tutta la rete in presenza di uno stimolo esterno applicato a un singolo sito.

\section{Il bursting e i neuroni dei generatori di moti a-semantici}

Ci stiamo occupando di come una rete di neuroni risponda collettivamente a uno stimolo. Dare un significato a una percezione vuol dire stimolare di conseguenza le aree motorie. Ma ci sono dei movimenti automatici (cardiaco, respiratorio, digestivo) che vanno eseguiti indipendentemente da stimoli esterni, in modo a-semantico. In questo caso non c'è convenienza a stare 'all'erta' in regime $\mathrm{HC}$, ma basta alternare raffiche (bursts) periodiche di spikes a intervalli quiescenti di riposo. Usando lo stesso sistema dinamico e spostando un parametro di controllo, un po' in su o in giù rispetto al regime di HC, si va ad un comportamento periodico (Fig. C13a) con frequenza che cresce con lo spostamento del parametro di controllo (c) o al contrario in regime quiescente (b).

Basandoci su questa informazione, possiamo far sì che un sistema con dinamica omoclinica alterni intervalli di raffiche periodiche con intervalli quiescenti, senza mai andare caotico. Basta aggiungere una modulazione a bassa frequenza rispetto alla frequenza delle spikes. Il comportamento è 
mostrato in figura C13. Possiamo considerare questo comportamento come metafora di quanto fanno i neuroni che pilotano il muscolo cardiaco. In regime normale abbiamo 60 battiti al minuto che consistono nell'alternanza di contrazioni muscolari con intervalli di riposo, come in figura 13b. Nel riposo notturno il ritmo scende a 45 battiti al minuto (Fig. 13a). Viceversa, sotto sforzo, possiamo avere 120 battiti al minuto (Fig. 13c).

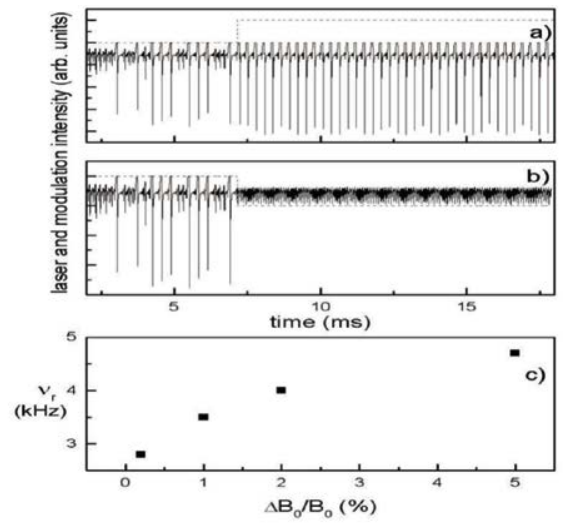

Figura C13 - Regimi periodico: a) quiescente; b) nel sistema con dinamica omoclinica, al variare di un parametro di controllo rispetto al regime $\mathrm{HC}$; c) nel caso periodico la frequenza cresce monotonamente con il parametro .

\section{Il controllo del caos come base dei processi linguistici}

Nella figura 23 della Parte A abbiamo visto come nasce il caos deterministico e nella figura 24 come lo si controlla; quest'ultima ha invocato una variazione di codice. Variazione di codice vuol dire rimpiazzare una dinamica deterministica di $N$ dimensioni con una a $N+m$ dimensioni. Questo salto è non-algoritmico in quanto $m$ varia da un individuo a un altro.

Questo suggerisce la definizione di coscienza $(C)$ come l'esplorazione dei vari $m$, scegliendo quello che ottimizza l'interazione con l'ambiente. Questo confronto richiede un linguaggio.

Dunque Cè distinta da consapevolezza percettiva (vedi più avanti la voce).

Codice cerebrale: Chi legge l'informazione codificata in una rete di neuroni - La funzione di Wigner rimpiazza l'homunculus

\section{La funzione di Wigner}

Avvertenza: la comprensione di questo paragrafo presuppone familiarità su integrali e trasformata di Fourier.

Abbiamo visto che l'informazione di un sistema HC è contenuta nella sequenza di spikes. Siccome ci interessa solo il tempo di accadimento di 
ciascuna, consideriamo la singola spike come una delta di Dirac, caratterizzata dal tempo di accadimento; il segnale neurale sarà dunque:

$$
f(t)=\sum \delta\left(t-t_{i}\right)
$$

L'informazione è nei tempi di arrivo delle spikes o nella loro separazione temporale (ISI, Inter-Spike-Interval):

$$
\{I S I\}=\left\{t_{i}-t_{i-1}\right\}
$$

Non possiamo pensare a un ipotetico homunculus che legga il segnale e decida. Se questo esistesse e avesse consistenza fisica, a sua volta avrebbe un sistema percettivo con un (homunculus dell'homunculus) che lo legge, e così via.

Cerchiamo più seriamente di estrarre l'informazione HC con componentistica fisica. Modo per esplorare $f(t)$ : ci si mette a $t$, si trasla il segnale a sinistra e a destra dello stesso tempuscolo $\tau / 2$; se fra i segnali traslati c'è qualche sovrapposizione, in corrispondenza della 'coincidenza' abbiamo un'area 1; contiamo tutti gli 1, e ripetiamo l'operazione per altre traslazioni. Se ci limitiamo a sommare le coincidenze parziali, abbiamo un conteggio globale che ci dice quante ISI uguali ci siano nella sequenza, ma non ci dice dove (a quali $t_{i}$ ) sono localizzate le spikes. Per avere questo 'dove', nel sommare distinguiamo ogni traslazione con un fattore di fase $\exp (i \omega \tau)$; avremo dunque come risultato:

$$
W(t, \omega)=\int f(t-\tau / 2) f(t+\tau / 2) e^{i \omega \tau} d \tau
$$

Dunque l'informazione completa è contenuta in una funzione bi-dimensionale, dove, si noti bene, $\omega$ non è il trasformato di Fourier di $t$, ma di $\tau$. La $W$ fu introdotta da Wigner nel 1932 in meccanica quantistica. Un aspetto peculiare della somma con fasi è che, se abbiamo una funzione che è la somma di due pacchetti a frequenza diversa e localizzati a tempi diversi, come in figura C14, $W$ non ha solo due regioni che sono le $W$ separate dei due pezzi, ma ha anche una regione intermedia con frange di interferenza.
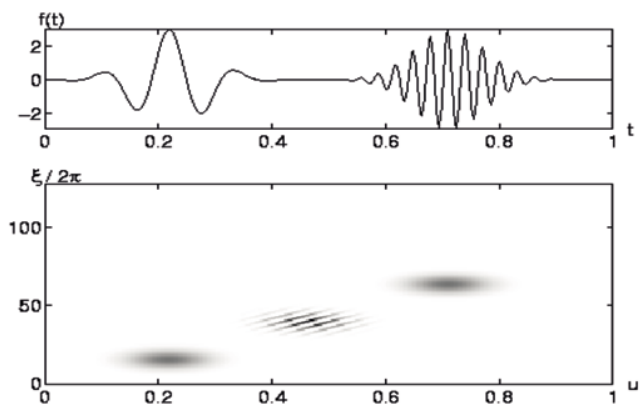

Figura C14 - In alto: segnale temporale $f(t)$ formato da due sinusoidi troncate a frequenze e tempi diversi. In basso: funzione di Wigner in 2D (asse orizzontale: tempo; asse verticale: frequenza). Oltre alle due zone separate spettanti alle due parti isolate, cề anche un segnale intermedio che è l'interferenza fra le due parti pesate dalla fase relativa. 
Questo nuovo strumento introduce una classe di misure non-locali che abbiamo chiamato 'codice temporale'. Quando, con una sonda locale ad altissima risoluzione, misuriamo il valore istantaneo $f(t)$ al tempo $t$, allora l'informazione su $f(t)$ si presenta come la parte alta della figura, che ci è familiare dalle introduzioni pedagogiche ai segnali. Il guaio è che una sonda con risoluzione infinita non esiste, e invece dobbiamo fare i conti con la strumentazione che abbiamo a disposizione: ciò implica ricorrere alla $W$ ed avere anche le interferenze fra le varie parti della sequenza che compongono il segnale.

La procedura chiamata da noi codice temporale non è limitata ai segnali cerebrali. In qualunque spartito musicale, rappresentiamo (anche se grossolanamente) l'intensità del suono in funzione di frequenza e tempo (Fig. C15).

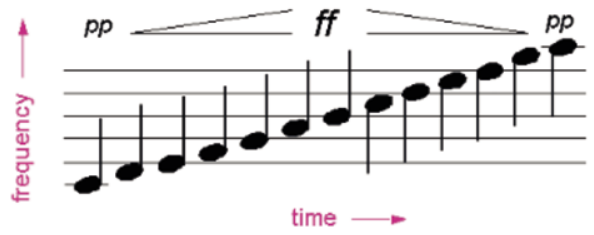

Figura C15 - Lo spartito musicale rappresenta la frequenza acustica al variare del tempo; la notazione pp (pianissimo) e ff (fortissimo) indicano il grado di intensità da associare ad ogni zona del piano 'frequenza-tempo'.

\section{Entropia delle percezioni: aspetti quantistici}

Ricapitoliamo i dati neurofisiologici: abbiamo treni di spikes di durata $200 \mathrm{~ms}$, fatti di spikes da $1 \mathrm{~ms}$ con separazione minima $3 \mathrm{~ms}$ e separazione media (legata alla banda $\gamma$ sui $40 \mathrm{~Hz}$ ) di $25 \mathrm{~ms}$. Se introduciamo delle finestre (bins) di $3 \mathrm{~ms}$, ciascuna avrà un impulso o sarà vuota: in codice binario, potremmo avere un numero di percezioni massimo $P_{M}$ pari a:

$$
2^{200 / 3} \cong 2^{66} \cong 10^{22}
$$

Ma non tutte le sequenze hanno uguale probabilità: sarà improbabile trovare $00000 \ldots$ oppure $11111 \ldots$ Pesando con la separazione media, si trova una entropia per unità di tempo pari a:

$$
\alpha=0,54
$$

che rappresenta un fattore riduttivo dell'esponente binario. Il numero di percezioni su $200 \mathrm{~ms}$ è allora:

$$
\mathrm{P}_{\mathrm{M}}=2^{\mathrm{S}}={ }^{20,54 \cdot 66} \cong 10^{11}
$$


Tenuto conto che al massimo abbiamo 5 percezioni distinte al secondo, e che l'arco della vita umana è sui $3 \cdot 10^{9} \mathrm{sec}$, pari a 90 anni, il numero massimo di percezioni immagazzinabili risulta $1,5 \cdot 10^{10}$, cioè dell'ordine della capacità calcolata. Data la grossolanità del calcolo, la cosa è soddisfacente, cioè l'evoluzione ci ha equipaggiato con un cervello adeguato alla durata della nostra vita, senza molti sprechi.

Supponiamo di troncare una percezione a un tempo $\Delta T<\bar{T}$. Introduciamo una indeterminazione $\Delta P$ nel numero di percezioni, pari al numero di tutte quelle percezioni i cui ISI sono identici fino a $\Delta T$ e che differiscono di almeno un bit nell'intervallo $\bar{T}-\Delta T$. Avremo:

$$
\Delta P=2^{\alpha(T-\Delta T)}=P_{M} \cdot 2^{-\alpha \Delta T}
$$

Approssimiamo questa relazione di incertezza con una iperbole tangente in un punto. Data la grande differenza fra esponenziale e iperbole, il valore che stiamo per calcolare è solo orientativo e varia molto con $\Delta T$. Abbiamo ad esempio:

$$
\triangle P \Delta T \equiv C=620 \text { parole } x \text { bins }
$$

Convertiamo in Js per confrontarci con $\hbar$.

Un bin corrisponde a $3 \mathrm{~ms}$. Un salto di parola corrisponde a un salto elementare da un attrattore caotico a uno vicino. Ricordiamo che abbiamo escluso una dinamica regolare per il singolo neurone, per evitare una energia di attivazione troppo alta, diciamo dell'ordine di $1 \mathrm{eV}$. Non esistono stime per il salto fra due attrattori caotici. Possiamo dire che l'energia deve essere maggiore di $k_{B} T$, altrimenti il salto avverrebbe spontaneamente e non ci sarebbero percezioni affidabili. Diamo come stima $10 k_{B} T$, pari a $0,25 \mathrm{eV}$. Moltiplicando per i $10^{8}$ neuroni della corteccia visiva, abbiamo:

$$
2,5 \cdot 10^{7} \mathrm{eV} \cong 3,6 \cdot 10^{-12} \mathrm{~J}
$$

Con questi fattori di conversione si trova:

$$
\Delta P \Delta T \equiv \mathrm{C}=6 \cdot 10^{-12} \mathrm{Js} \cong 10^{22} \mathrm{~h}
$$

È questo il quanto nel codice percettivo. Si noti che non c'entra niente con $\hbar$, con buona pace di chi (come Penrose) ha cercato di individuare una dinamica quantistica microscopica nel cervello.

Si noti che l'unico requisito è stato un misuratore non locale; niente di legato a coscienza ecc.: pertanto si può progettare una rete di siti $\mathrm{HC}$ collegati e da leggere con un codice di Wigner.

\section{Complessità}

Nella figura 8 della Parte A, abbiamo introdotto la differenza fra complessità algoritmica e complessità semantica. 
La prima è misurata dalla lunghezza in bit del programma che costituisce l'algoritmo del Bayes diretto; la chiameremo anche complicazione.

La seconda, legata alle operazioni linguistiche, indica quanti nuovi significati attribuiamo allo stesso termine linguistico ed è la base del Bayes inverso.

In figura $\mathrm{C} 16$ la complessità algoritmica, vista in due definizioni differenti, è rappresentata per un sistema caotico al ridursi del tempo di memoria (cioè, all'aumentare di $K$ ).

Se chiamiamo $t$ il tempo necessario per descrivere un singolo elemento e se abbiamo $N$ elementi, il tempo totale di calcolo $T$ dipende se gli $N$ elementi sono disgiunti o mutuamente accoppiati; avremo rispettivamente:

caso semplice: $T=N t_{1}$

caso interconnesso: $T^{1}=10^{\mathrm{N}} t_{1}$

Per esempio, sia $t_{1}=1$ microsec $=10^{-6} \mathrm{sec} ; N=100$, allora:

$1^{\circ}$ caso: $T=100 t_{1}=10^{2-6}=10^{-4} \mathrm{sec}$;

$2^{\circ}$ caso: $T^{\prime}=10^{100-6}=10^{94} \mathrm{sec}$

(molto più lungo dell'età dell'universo che è $10^{17} \mathrm{sec}$ ).

Due definizioni di complessità: è quantificabile ma ha ridotto il mondo a un solo codice di lettura; la diremo piuttosto complicazione.

1. C. algoritmica (CA) (G.J. Chaitin, 1965): n. di bits del programma più corto che, partendo da una memoria vuota, calcola una stringa e poi termina.

Max CA di una stringa di $\mathrm{n}$. bits $=\mathrm{n}$ (caso random)

2. Spessore (D: depth) logico (C.H. Bennett, 1986): tempo di esecuzione del programma più corto.

1)

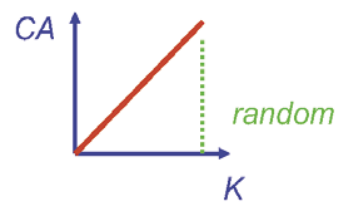

2)

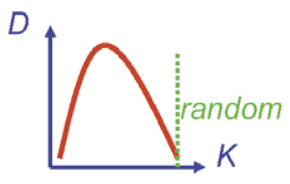

Figura C16 - Due diversi aspetti della complessità algoritmica per un sistema caotico, in funzione di $K$ (entropia di Kolmogorov = inverso del tempo di perdita dell'informazione iniziale). In 1), la $C A$ di Chaitin cresce linearmente con $K$. In 2), si presenta lo spessore logico $D$ introdotto da C.H. Bennett e che è un indicatore della ricchezza semantica del sistema. 


\section{Complicazione e complessità}

Cambiando codice si passa da una descrizione atomistica, in cui la complessità è solo complicazione, a una collettiva, che riduce la complicazione

Chiameremo complessità semantica la ricchezza di significato (ambito di applicabilità) dei nuovi concetti.

Nella figura 25 della Parte A, abbiamo mostrato alcuni esempi di come una scienza creativa operi con l'introduzione di nuovi concetti.

Nella figura 19 della Parte A, abbiamo scomposto una mela in affezioni quantitative (sapore, odore, colore ecc.). La collezione dei numeri può bastare per il rifornimento di un mercato, però non esaurisce la realtà della mela. In effetti l'intero (la mela) precede le misure separate, che forniscono quantità derivate, non primitive: si appoggiano alla mela ma, una volta enucleate (astratte), non permettono la ricostruzione logica della mela. Non siamo mai sicuri che non ci sia un ulteriore punto di vista inedito che arricchisca la descrizione della mela.

Punto di vista ecologico (secondo la dizione di G. Bateson): la complessità come apertura all'ambiente:

a) noise o

b) influsso del mondo esterno?

a) Sporcare un calcolo deterministico con un disturbo che permetta di esplorare situazioni altrimenti non accessibili è una strategia dell'AI; compare con diversi nomi: oracle machine, simulate dannealing, macchine di Boltzmann.

b) Invece il problema semantico è: possiamo acquisire cose significative del mondo attraverso l'approccio linguistico?

\section{Consapevolezza percettiva e NCC (Neural Correlates of Consciousness)}

Applicando molti elettrodi sullo scalpo si può registrare dall'esterno l'attività elettrica sotto forma di EEG (elettro-encefalo-grammi): la risoluzione temporale è buona (al millisec), ma spazialmente si raccolgono $\mathrm{i}$ potenziali provenienti da regioni vicine e non necessariamente correlate, cosicché il segnale EEG è molto 'sporco' e sembra affetto da rumore.

Una tecnica alternativa di visualizzazione dell'attività cerebrale è la f-MRI (functional Magnetic Resonance Imaging), che è sensibile al flusso sanguigno e all'ossigenazione del sangue; il contrasto magnetico è dovuto al ferro nell'emoglobina.

La f-MRI ha scarsa risoluzione spazio-temporale; va bene per localizzare collettivamente aree cerebrali impegnate nello stesso compito. Lo stesso dicasi per la PET (Positron Emission Tomography), che misura la concentrazione nei tessuti di radio-isotopi immessi nel sangue. Entrambi i metodi si basano sul maggior afflusso di sangue in aree impegnate in un compito.

Nelle figure C17 e C18 si riportano i risultati di una indagine EEG in soggetti umani (1999: gruppo di F. Varela a Parigi). A partire dal tempo $t=0$ si mostra una immagine significativa (un profilo femminile) e una 
non significativa (lato sinistro della figura) e si registra l'attività EEG da tutti gli elettrodi, selezionando in particolare la cosiddetta banda $\gamma$ attorno a $40 \mathrm{~Hz}$ (separazione media di $25 \mathrm{msec}$ fra le spikes).

A destra della figura C17 si nota che l'EEG si attiva attorno ai $200 \mathrm{~ms}$ e poi a $800 \mathrm{~ms}$; la differenza fra i due casi sembra solo quantitativa e non qualitativa. Per capire di più, si esplorano le correlazioni di fase fra i segnali provenienti dai vari elettrodi (Fig. C18) e si vede che:

- nel caso significativo, a $200 \mathrm{~ms}$ c'è una forte correlazione fra la zona occipitale corrispondente all'area visiva e la zona PFC (corteccia prefrontale); nel caso non significativo, manca questa correlazione di fase; - a 800 ms c'è correlazione fra PFC e aree motorie e linguistiche in ambo i casi.

Interpretazione (si veda lo schizzo di sezione cerebrale in cima a Fig. C17): la PFC è il centro decisionale, che raccoglie l'informazione sensoriale, la elabora nei $600 \mathrm{~ms}$ che intercorrono fra l'arrivo a PFC e il suo collegamento con aree motorie e poi spedisce i comandi per azioni. Queste sono: catturare una preda o cibo, sfuggire a un predatore (e fin qui vale per tutti gli animali) o parlare di quanto visto (e questo sembra specifico degli umani).

- Occipite= Corteccia
visiva(200ms)
$-P F C=$ corteccia pre-
frontale (centro
decisionale)
-Aree motorie e
linguistiche $(800 \mathrm{~ms})$
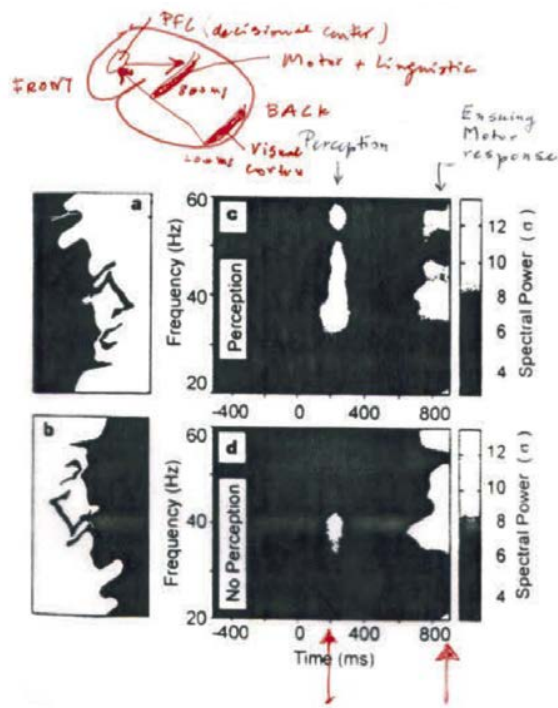

Figura C17 - Risposta transitoria di un soggetto umano esposto a una immagine significativa (in alto) e non significativa (in basso) applicate al tempo $t=0$. Dopo $200 \mathrm{~ms}$ l'EEG dà segnale sulla banda $\gamma$ (centrata sui $40 \mathrm{~Hz}$ ). Dopo tace fino a $800 \mathrm{~ms}$, quando ritorna. A parte differenze quantitative, non sembra che ci siano differenze qualitative fra i due casi. Ma la figura seguente (vedi didascalia) giustifica i fatti riassunti nello schema riportato in alto: a $200 \mathrm{~ms}$ la corteccia visiva posta nelloccipite si collega con la PFC (Pre Frontal Cortex = corteccia pre-frontale) che è un centro decisionale; dopo altri $600 \mathrm{~ms}$, spesi in elaborazione, la PFC si collega con le aree motorie. 
Chiamiamo NCC (Neural Correlates of Consciousness = correlati neuronali della consapevolezza percettiva, non della coscienza!) gli accordi di fase di figura C18.

I dettagli qui illustrati sono la base della percezione, che nella Parte A abbiamo presentato in modo globale mediante l'inferenza di Bayes.

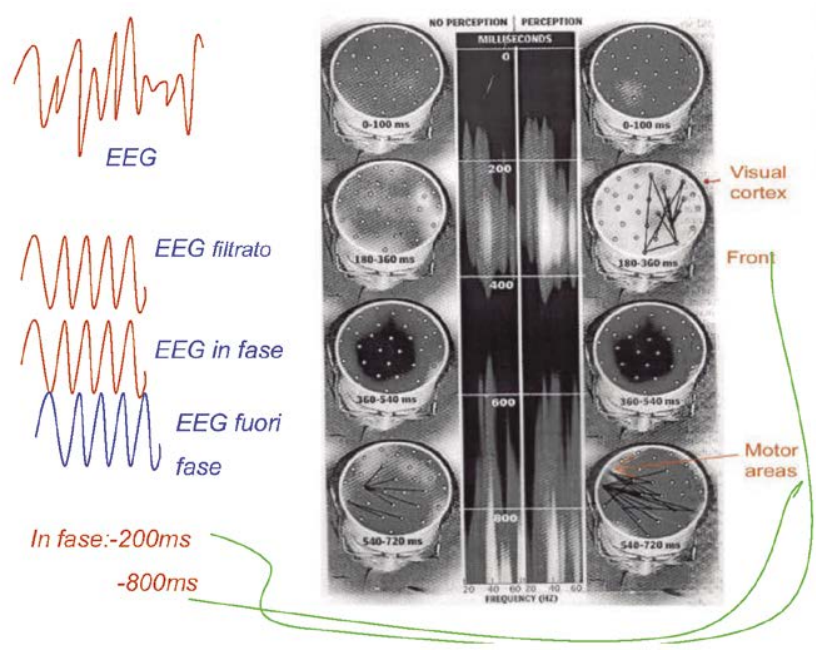

Figura C18 - L'EEG è catturato da diecine di elettrodi incollati allo scalpo. Nella figura si mostra il cranio del soggetto (fronte verso il basso, come risulta dagli occhiali). I cerchietti indicano gli elettrodi. A sinistra caso a-semantico, a destra immagine significativa. In ambo i casi la banda $\gamma$ si accende a 200 e $800 \mathrm{~ms}$. Ma analizzando in dettaglio i segnali dei vari elettrodi, a $200 \mathrm{~ms}$ cè a destra una correlazione di fase fra corteccia visiva e PFC (linee che collegano elettrodi distanti); questa correlazione è assente a sinistra. Interpretazione: non cè trasferimento di informazione significativa alla PFC e l'accensione della banda $\gamma$ è incoerente. Invece a $800 \mathrm{~ms}$ in ambo i casi scatta una correlazione; interpretazione: la PFC attiva l'area linguistica per dire quel che vede.

\section{Delta di Dirac}

Consideriamo una funzione del tempo $y(t)$ e pesiamone il tratto tra $t_{1}$ e $t_{2}$ con una funzione peso di area 1 . Estrarremo il valore intermedio $y_{0}$ con probabilità $P\left(y_{0}\right)$ spalmata su tutto l'intervallo $\left(t_{1}-t_{2}\right)$.

Se ora restringiamo la funzione peso attorno a $t_{0}$, avremo una curva di probabilità più stretta attorno al valore $y_{0}$.

La delta di Dirac è il limite della funzione peso (sempre di area 1), quando l'intervallo $\left(t_{1}-t_{2}\right)$ attorno a $t_{0}$ si riduca a zero (e pertanto il picco della funzione peso tende ad infinito per mantenere area unitaria alla funzione peso) (Fig. C19). 


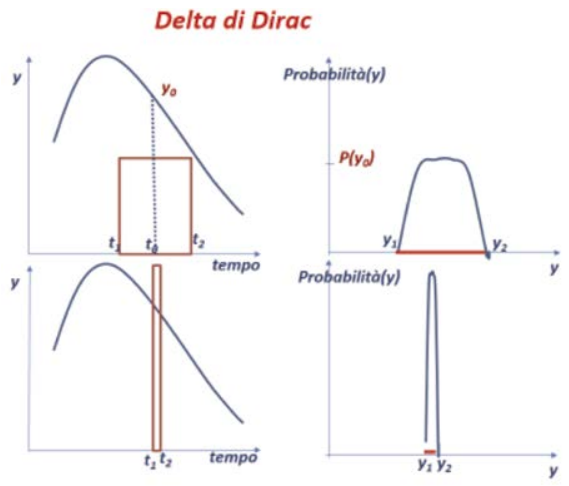

Figura C19 - Pesiamo una funzione $y(t)$ con due pesi di area 1 ma di durata diversa. Nel secondo caso, la curva di probabilità attorno al valore $P\left(y_{0}\right)$ si restringe. Al limite di peso infinitamente stretto, $t_{2}=t_{1}$ (delta di Dirac) si ha una probabilità centrata sul punto $y_{0}$ e senza sparpagliamento attorno (vedi Certezza e figura 21 della Parte A).

\section{Dimensioni frattali}

Partiamo da un segmento di lunghezza 1 e dividiamolo in 3 tratti. Asportiamo la parte centrale e ripetiamo l'operazione nei tratti restanti. Continuando così, costruiremo l'insieme di Cantor. Se invece rimpiazziamo la parte centrale con gli altri due lati di un triangolo equilatero, e continuiamo così, otteniamo la curva di Koch.

Ricopriamo ciascuno di questi oggetti con $N$ sfere di raggio $r$, dove $r$ è tale da assicurare che non si perda risoluzione ad ogni passo della partizione. All'aumentare del numero di partizioni, la dimensione frattale $D$ definita come:

$$
D=\frac{\log N}{\log (1 / r)}
$$

è un invariante il cui significato appare dalla figura C20.

$$
\begin{aligned}
& \text { Cantor } \\
& \text { Segmento } \\
& \text { Koch } \\
& r=1 \\
& \stackrel{1}{0 \quad 1}
\end{aligned}
$$

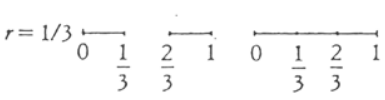

$$
\begin{aligned}
& r=1 / 9 \underset{0 \frac{1}{9}}{\longrightarrow} \\
& -\mathfrak{1} \\
& \stackrel{0 \frac{1}{9} \cdots \frac{8}{9}}{1} \\
& \overbrace{\frac{1}{9}}^{\frac{1}{3}} \underbrace{\frac{2}{3}}_{\frac{8}{9} 1} \\
& D=\frac{\log 2}{\log 3} \\
& D=\frac{\log 3}{\log 3} \\
& D=\frac{\log 4}{\log 3} \\
& =0.6309 \\
& =1 \\
& =1.2618
\end{aligned}
$$

Figura C20 - Successive partizioni che generano: il set di Cantor $(D=0,6309)$; il segmento euclideo unitario $(D=1)$; la curva di $\operatorname{Koch}(D=1,2618)$. 


\section{Entanglement (intreccio), computazione quantistica e decoerenza (per- dita aspetti quantistici)}

Parole chiave della fisica quantistica sono:

- entanglement (intreccio): due sistemi possono essere in stati strettamente correlati; lo stato quantico dell'insieme dipende dallo stato di ciascun sistema, anche se essi sono spazialmente separati;

- computazione quantistica, fatta simultaneamente su tutti gli stati entangled: velocizza il calcolo;

- decoerenza: al passare del tempo, due sistemi entangled - disturbati dall'ambiente - perdono l'entanglement, diventando distinguibili $(\rightarrow$ fisica classica).

\section{Epigenetica}

In biologia, il fenotipo è determinato non tanto dal genotipo, quanto dalla sovrapposizione al genotipo stesso di un'impronta' che ne influenza il comportamento funzionale. Un segnale epigenetico è un cambiamento ereditabile che non altera la sequenza nucleotidica di un gene, ma la sua attività.

Dunque, epigenetica denota attività del gene non fissata una volta per tutte, come l'algoritmo in Bayes, ma regolata dal confronto/scontro fra il gruppo $h^{*}$ di competenze accumulate e l'ultimo invasore $d$ (virus o altra perturbazione ambientale); pertanto $P(d \mid h)$ emerge come in Bayes inverso.

In epigenetica, non si modifica la sequenza delle basi del DNA del gene, ma se ne controlla l'attività o intercalando una molecola estranea (metilazione) o variando la curvatura della catena di DNA (acetilazione); si modifica così l'interazione con altre molecole, cioè si altera l'espressione' del gene.

\section{Equilibrio e stabilità}

Osserviamo il pendolo della figura C21.

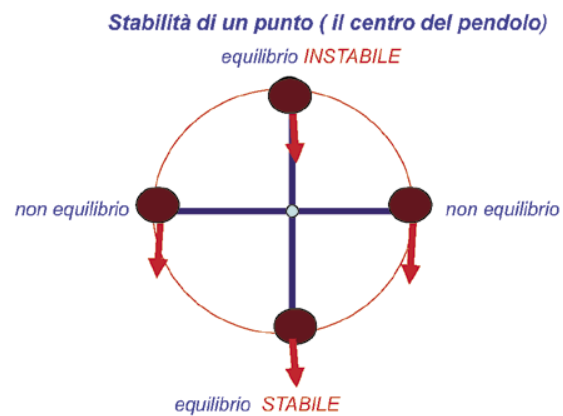

Figura C21 - Un pendolo è composto da un peso (la palla nera) attaccato ad un'asta rigida che è ancorata dall'altra parte. Nelle due posizioni orizzontali, non cè equilibrio, perché la forza peso (rappresentata da una freccia) tende a portare la palla in giù. Nelle due posizioni verticali, c’è equilibrio. 
L'equilibrio è stabile nella posizione bassa: per qualunque piccolo spostamento la forza che nasce riporta il peso nella posizione iniziale; è instabile nella posizione alta: per qualunque spostamento nasce una forza che tende ad amplificare lo spostamento.

\section{Forze lineari e non lineari}

La dizione forza lineare e non lineare fa riferimento alla dipendenza della forza dalla posizione, come mostrato rispettivamente nelle figure 36 e 38 della Parte B; la nozione è qui ripresa in figura C22.

In presenza di molte forze, nel caso lineare basta studiare separatamente la dinamica sotto ciascuna di esse e poi sommarne i contributi (sovrapposizione degli effetti); nel caso non lineare, le mutue interazioni sono inscindibili e vanno studiate insieme.

Pertanto, studiando la dinamica nell'approssimazione lineare, bastava studiare il singolo grado di libertà. Bisogna arrivare a Poincaré (1890) e allo studio di forza non lineare per scoprire che da 3 gradi di libertà in su si hanno le instabilità trasversali delle traiettorie che oggi chiamiamo caos deterministico (Fig. 23, Parte A).

A forze non lineari corrispondono paesaggi dell'energia complessi, chiamati in biologia 'paesaggi epigenetici', come mostrato in figura C23.

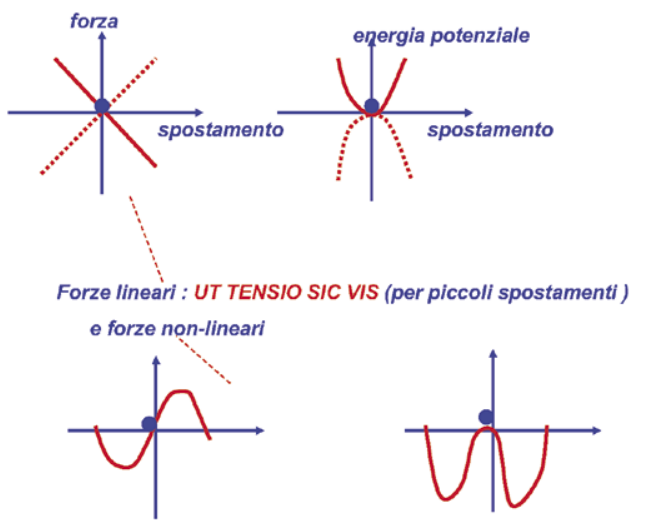

Figura C22 - In alto forza ed energia nel caso stabile (linea intera) e instabile (linea tratteggiata); in ogni caso, la forza in funzione dello spostamento è una retta e l'energia è una parabola (dinamica lineare). In basso: un esempio di forza non lineare e della corrispondente energia potenziale.

Nella figura 23 della Parte A abbiamo mostrato che, mentre in dinamica lineare si considerano gradi di libertà separati e poi li si somma (sovrapposizione degli effetti), in dinamica non lineare bisogna valutare il sistema nella sua globalità e da 3 gradi di libertà in su nasce il caos deterministico. 

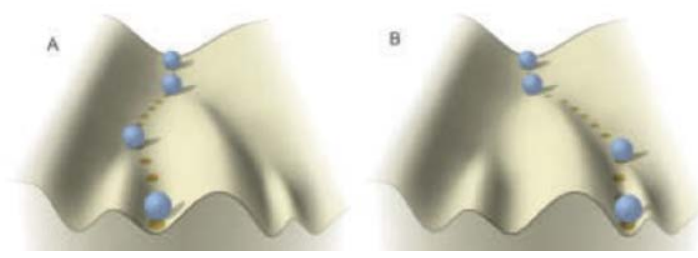

Figura C23 - Paesaggio epigenetico di Waddington; relazione fra genotipo e fenotipo. Un organismo (la palla) si muove attraverso valli che rappresentano vari stati fenotipici. La forma è determinata dal genotipo. Processo di sviluppo in due individui (A e B) con lo stesso genotipo (come in gemelli monozigotici). Differenti punti finali fenotipici determinati dal caso e da effetti ambientali. [Da: Mitchell, 2007: 113]

\section{Galileo Galilei}

Galileo era arrivato a costruire un cannocchiale da venti ingrandimenti che usò per guardare le stelle.

«[...] mi procurai un tubo di piombo, alle cui estremità adattai due lenti, una convessa e l'altra concava; accostando poi l'occhio alla concava, scorsi gli oggetti abbastanza grandi e vicini».

Nel 1609, Galileo scoprì che sulla Luna mancavano le nubi e che la superficie era tormentata da crateri e picchi altissimi. Il 7 gennaio 1610 vide che Giove era in mezzo a tre stelline, disposte in linea retta. Continuò l'osservazione. Il numero di stelline, il 13, salì a quattro. Notò che si trovavano a volte da una parte, a volte dall'altra rispetto a Giove. Arrivò alla conclusione che dovevano essere satelliti di Giove con orbite regolari. Espose queste scoperte nel Sidereus nuncius, un libricino di poche decine di pagine, scritto e pubblicato nel giro di qualche settimana.

Scoprì le fasi di Venere. Vide che Saturno aveva misteriose appendici, ossia era «tricorporeo» (si trattava degli anelli che il suo cannocchiale non era in grado di distinguere). Vide che il Sole aveva macchie sulla superficie e dal loro spostamento dedusse che l'astro ruota su se stesso.

Tolomeo e Copernico consideravano l'astronomia una scienza geometrica: fu Galileo a farne una scienza fisica, su corpi reali.

Il metodo di Galileo si fonda su due pilastri:

- sensate esperienze (resoconti di esperimenti, osservazioni della natura) compiute con i sensi potenziati con strumenti, come il telescopio e l'orologio;

- necessarie dimostrazioni, in un teorema, se concordo con le premesse e capisco la dimostrazione, devo per forza accettare la conclusione.

Anche Bacone proponeva sensate esperienze, registrate in tavole, e necessarie dimostrazioni utilizzando la logica, attraverso l'esclusione di alcune ipotesi. La differenza sta nell'uso della matematica: se l'ipotesi è espressa da una formula matematica, le conseguenze risultano semplicemente usando il formalismo (algoritmo). 
Inoltre, si ricerca nel mondo, non sui testi altrui:

[...] ma gli ingegni vulgari timidi e servili, che altrettanto confidano, sopra l'autorità di un altro, quando vilmente diffidano del proprio discorso, né più oltre credono che si estenda l'obbligo loro, che a interpretare i detti di un altr'uomo, rivolgendo notte e giorno gli occhi intorno ad un mondo dipinto sopra certe carte, senza mai sollevargli a quello vero e reale, che, fabbricato dalle proprie mani di Dio, ci sta sempre aperto innanzi (1632, Dialogo sopra i due massimi sistemi del mondo, corsivi miei)

La filosofia è scritta in questo grandissimo libro, che ci sta aperto innanzi agli occhi (io dico l'universo), ma non si può intendere se prima non s'impara a intendere la lingua, e conoscer $i$ caratteri, ne' quali è scritto. Egli è scritto in lingua matematica, e i caratteri son triangoli, cerchi ed altre figure geometriche, senza i quali è impossibile a intenderne umanamente parola (Il Saggiatore, corsivi miei).

Il mondo dell'esperienza è scisso in due: da una parte gli aspetti quantitativi, traducibili in linguaggio matematico, e perciò oggettivi; dall'altra gli aspetti qualitativi, non riducibili ad un rapporto meccanico tra i nostri organi di senso e il mondo esterno, e pertanto da considerare soggettivi.

\section{Gene}

Il gene è l'unità ereditaria fondamentale degli organismi viventi. I geni sono porzioni localizzate all'interno della sequenza di DNA e contengono le informazioni per codificare una proteina. Il gene presenta una diversa composizione a seconda che si considerino organismi procarioti o eucarioti: nei primi è costituito da sequenze codificanti, nei secondi contiene anche sequenze non codificanti. Nel gene eucariotico la sequenza codificante si definisce esone e quella non codificante introne (Fig. C24).

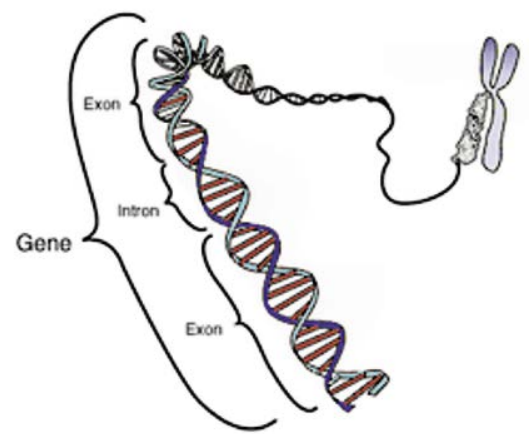

Figura C24 - Relazione tra gene, DNA e cromosoma. Gli introni sono regioni non codificanti nei geni eucarioti, solo gli esoni codificano proteine. 


\section{Gerarchia di scale}

È opportuno aver presenti le scale degli oggetti dell'Universo, sia in lunghezze (espresse in metri), sia in massa (espresse in kg) (Fig. C25).

Gli oggetti più grossi sono composti di quelli a scala inferiore, nella visione riduzionistica (bottom-up): ciò dovrebbe bastare a stabilirne le proprietà.

Invece, come abbiamo visto alla voce вотTOM-UP E TOP-DOWN, gli stadi più alti possono influenzare per feedback gli stadi più bassi. Esempi cospicui: l'epigenetica in biologia e il linguaggio nella cognizione.

\section{Gerarchia di scale}

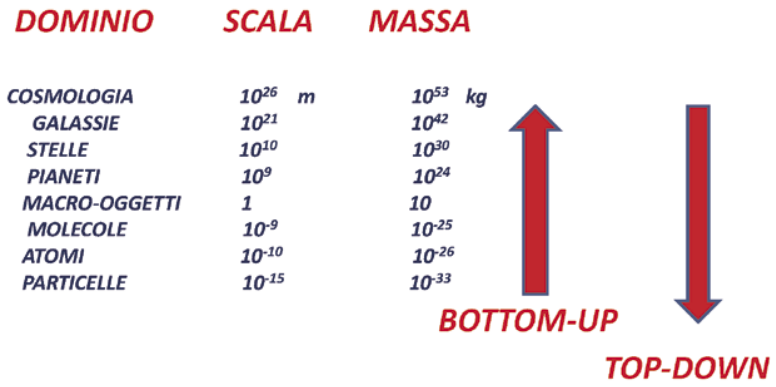

Figura C25 - Gerarchia di scale fra gli oggetti dell'Universo.

\section{GWS (Global Work Space)}

La teoria cognitiva dello Spazio di Lavoro Globale (Global Work Space) di Bernard Baars (1988) è un modello di coscienza, intesa come consapevolezza del mondo esterno, fornita dagli organi di senso e organizzata in una rappresentazione integrata di tutte le sue proprietà percettive (il cosiddetto binding). Essa permette:

- l'integrazione di informazioni multimodali (provenienti cioè da tutti i sensi) e dei processi cognitivi superiori;

- i processi di focalizzazione dell'attenzione;

- la ricerca, l'apprendimento, la codifica e l'adattamento a informazioni nuove;

- l'accesso e la gestione volontaria dei processi esecutivi e di controllo;

- i processi decisionali e di risoluzione dei problemi;

- il raggiungimento di scopi complessi e la correzione degli errori.

Il sistema cognitivo è costituito da un insieme di moduli e circuiti separati con funzioni specifiche. Non tutti questi circuiti portano però alla coscienza, la quale emerge solo in seguito ad una loro particolare interazione. Un Global Work Space è in sostanza uno spazio di scambio di in- 
formazioni che permette l'interazione di questi sistemi (o moduli) inconsci specializzati del cervello.

Lamplificazione attenzionale top-down è il meccanismo per mezzo del quale questi processori modulari sono temporaneamente reclutati e accedono al Global Work Space. Questa attenzione top-down fa sì che le aree necessarie si attivino simultaneamente e, con un'elevata sincronicità, oscillino nella banda di frequenza gamma $(40-70 \mathrm{~Hz})$.

La figura C26 visualizza i meccanismi sopra elencati; la figura C27 dà un possibile modello operativo, in termini di sincronizzazione di impulsi neuronali; questi comportamenti sono riassunti dalla visione sintetica della percezione come inferenza di Bayes.

\section{Dinamica del GWS (global workspace)}

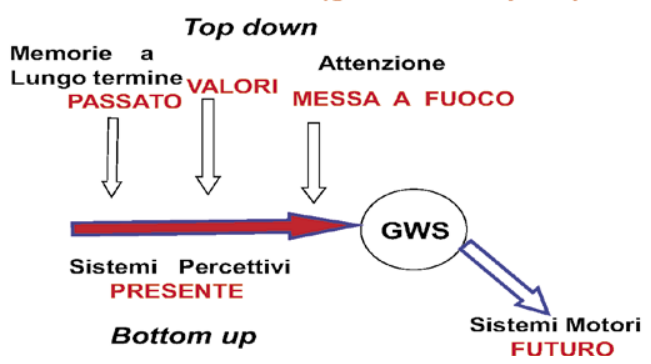

Figura C26 - I sistemi percettivi non sono modulati solo dai sensi, ma ricevono segnali da memorie pregresse, sono influenzati da valori e reinterpretati dall'attenzione; una volta letti dal GWS, ne emerge una decisione motoria. Lo stimolo esterno (bottomup), modificato dalle ipotesi interpretative (top-down).

Implementazione dinamica del Global Workspace

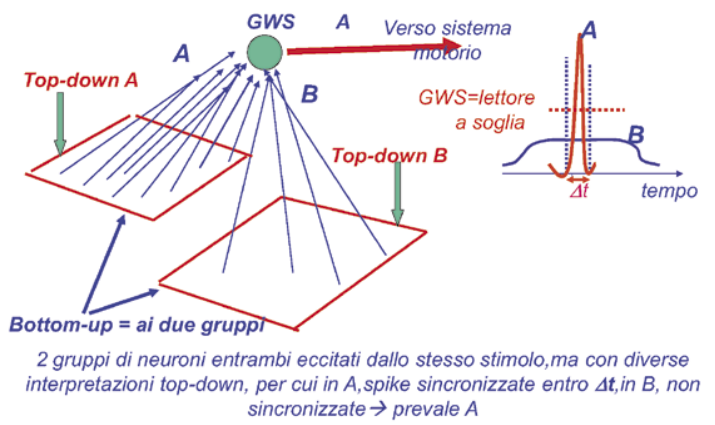

Figura C27 - Competizione fra due aree neuronali, entrambe collegate col GWS. A parità di stimolo esterno (bottom-up), due diverse perturbazioni top-down, A e B rispettivamente, determinano gradi diversi di sincronizzazione (es.: alta in $\mathrm{A}$, bassa in $\mathrm{B}$ ). Si può ritenere il GWS come un lettore a soglia che opera per un tempo limitato $\mathrm{D} t$. Nel caso A, i segnali sincronizzati si sommano e danno un impulso che va sopra soglia. Nel caso B, invece, solo pochi impulsi cadono entro $\mathrm{D} t$ e pertanto il segnale di B resta sotto soglia. 


\section{Macchina di Turing}

Sorta di computer ideale corredato di un programma da eseguire, la macchina di Turing ha un funzionamento che può essere facilmente descritto in termini matematici, facendo uso di concetti come insieme, relazione e funzione.

È stato dimostrato che un certo problema può essere risolto da un computer (opportunamente programmato) se e solo se esso può essere risolto anche da una macchina di Turing. Oltre alla macchina di Turing, proposta nel 1936, nello stesso periodo altri matematici hanno elaborato diverse rappresentazioni formali del concetto di algoritmo, fra i quali ricordiamo, per esempio, il lambda calcolo. Dopo alcuni anni, emerse che tutti questi modelli erano equivalenti.

$\mathrm{Da}$ questi risultati scaturì la tesi di Church-Turing, che afferma che qualsiasi algoritmo sia modellabile con una macchina di Turing. In altri termini, questa tesi sostiene che è sostanzialmente impossibile cercare di immaginare un modello di algoritmo più potente e, di conseguenza, che nessuna macchina potrà mai risolvere problemi che una macchina di Turing non possa risolvere in linea di principio.

Dalla definizione di algoritmo si evincono alcune proprietà necessarie:

- i passi costituenti devono essere 'elementari', ovvero non ulteriormente scomponibili (atomicità);

- i passi costituenti devono essere interpretabili in modo diretto e univoco dall'esecutore, sia esso umano o artificiale (non ambiguità);

- l'algoritmo deve essere composto da un numero finito di passi e richiedere una quantità finita di dati in ingresso (finitezza);

- l'esecuzione deve avere termine dopo un tempo finito (terminazione);

- l'esecuzione deve portare a un risultato univoco (effettività).

Così, ad esempio, 'rompere le uova' può essere considerato legittimamente un passo elementare di un 'algoritmo per la cucina' (ricetta), ma non potrebbe esserlo anche 'aggiungere sale quanto basta' dato che l'espressione 'quanto basta' è ambigua, e non indica con precisione quali passaggi servano per determinare la quantità necessaria.

L'algoritmo viene generalmente descritto come 'procedimento di risoluzione di un problema'. In questo contesto, i 'problemi' che si considerano sono quasi sempre caratterizzati da dati di ingresso (input) variabili, su cui l'algoritmo stesso opererà per giungere fino alla soluzione.

Le proprietà di un algoritmo sono le seguenti.

- Non ambiguità: le istruzioni devono essere univocamente interpretabili dall'esecutore dell'algoritmo.

- Eseguibilità: l'esecutore deve essere in grado, con le risorse a disposizione, di eseguire ogni istruzione in un tempo finito.

- Finitezza: l'esecuzione di un algoritmo deve terminare in un tempo finito per ogni insieme di valori in ingresso. 


\section{Meme}

R. Dawkins ha introdotto il termine meme nel libro Il gene egoista, per descrivere una unità base dell'evoluzione culturale umana analoga al gene, in base all'idea che il meccanismo di replica, mutazione e selezione, che caratterizza l'evoluzione darwiniana, si verifichi anche in ambito culturale. Così come in biologia, la presenza di questi elementi porta all'emergere spontaneo di effetti evolutivi. Dawkins descrive il meme come una unità di informazione residente nel cervello: si tratta di uno schema che può influenzare l'ambiente in cui si trova (attraverso l'azione degli uomini che lo portano) e si può propagare (attraverso la trasmissione culturale).

Un meme può essere parte di un'idea, così come può essere una melodia, una forma, un'abilità, un valore morale o estetico; può essere in genere qualsiasi cosa possa essere imparata e trasmessa ad altri come un'unità. $\mathrm{Al}$ pari dell'evoluzione genetica, anche l'evoluzione memetica non può avvenire senza mutazioni. La mutazione produce varianti, ma solo le più adatte si replicano: esse diventano più comuni ed aumentano la loro capacità di replicarsi ulteriormente.

Il meme è dunque un'unità di trasmissione culturale che permette alla cultura umana di evolversi e tramandarsi di cervello in cervello, di generazione in generazione.

Nella teoria del gene egoista gli individui viventi sono macchine che $i$ geni utilizzano per garantire la propria sopravvivenza. Allo stesso modo anche un meme è 'interessato' solamente alla propria sopravvivenza. $I$ cervelli sono contenitori e l'imitazione è il sistema di diffusione. In questo modo i memi sopravvivono, aldilà degli individui che li ospitano in un determinato momento.

\section{Memoria}

La memoria è un magazzino all'interno del quale l'individuo può conservare tracce della propria esperienza passata, cui attingere per riuscire ad affrontare situazioni di vita presente e futura. Atkinson e Shiffrin (1968) postularono l'esistenza di tre tipi di memoria: memoria sensoriale, memoria a breve termine $(\mathrm{MBT})$ e memoria a lungo termine (MLT).

La memoria sensoriale mantiene le tracce acquisite solo per qualche secondo, il tempo sufficiente per percepire il mondo esterno. La memoria a breve termine (MBT) contiene le informazioni per un periodo di tempo molto breve, sotto la decina di secondi. Dopo questo tempo, la traccia decade. Una delle caratteristiche di questo magazzino è contenere contemporaneamente poche unità di informazioni: infatti, in un soggetto adulto le unità contenibili nella MBT sono cinque più o meno due, variano a seconda delle caratteristiche del materiale da ricordare. Se queste informazioni non sono trasferite nel magazzino a lungo termine, decadono 
e spariscono. La MBT svolge una funzione transitoria e di servizio tra la memoria sensoriale e la memoria a lungo termine.

La memoria a lungo termine (MLT) è un archivio avente capacità quasi illimitata, dove sono conservate tutte le esperienze e le conoscenze acquisite nel corso della vita e quelle che corrispondono al nostro carattere o temperamento.

\section{Memoria a lungo termine}

La MLT si suddivide in memoria esplicita, o dichiarativa, e memoria implicita, o procedurale.

La memoria esplicita comprende tutto ciò che può essere descritto consapevolmente dal soggetto ed è suddivisa in memoria episodica, memoria semantica e memoria emozionale. La memoria dichiarativa è suddivisa in memoria semantica, che riguarda conoscenze generali sul mondo esterno, ed episodica, che riguarda specifici episodi e le loro circostanze.

La memoria procedurale o implicita, al contrario, contiene abilità motorie, percettive e cognitive.

Dunque, mentre la memoria dichiarativa riguarda tutte le conoscenze esplicite (esprimibili a parole) che si hanno sul mondo, la memoria procedurale non è verbalizzabile, e riguarda il fare qualcosa, come l'andare in bicicletta.

\section{Memoria a breve termine}

1. Memoria sensibile (durata inferiore a $3 \mathrm{sec}$ ): isola pre-semantica in cui non ci si pone problemi di significato. All'interno, si 'aggiustano' canali sensori con diversi tempi caratteristici (ad esempio, acustico e visivo).

2. Memoria a breve termine o working memory (WM): versione filtrata della memoria sensibile, durata fino a 15-30 sec. In questo intervallo avviene il confronto fra isole diverse. Ci sono tre parti di WM:

a) fonologica, si spegne in 2 sec se non esercitata;

b) visuo-spaziale;

c) esecutiva, che coordina le altre due.

Nel cervello esistono due diversi metodi di immagazzinare informazioni per dare vita alla 'memoria': il potenziamento a lungo termine e il potenziamento a breve termine. Il secondo consiste in una alterazione temporanea delle sinapsi coinvolte, che vengono iper-sensibilizzate mediante la sintesi di nuovi recettori di membrana. Il potenziamento a lungo termine, invece, richiede più tempo e coinvolge l'attivazione di proteine che modificano l'espressione genica, e, in ultima analisi, rinforzano in maniera molto più duratura la sinapsi.

La struttura cerebrale principale coinvolta nella memoria è l'ippocampo: nelle cellule dell'ippocampo è stato verificato, in vivo, il processo di potenziamento a lungo termine. 


\section{Moti oculari nel linguaggio figurativo}

Il teorico dell'estetica del Settecento, Gotthold E. Lessing, nel suo Laocoonte criticava Orazio, il quale aveva detto che la "pittura è come la poesia». Invece, per Lessing, la poesia non è accostabile alla pittura poiché opera nel 'tempo', mentre le arti figurative operano nello 'spazio', devono raffigurare i corpi e le realtà sensibili di questi nello spazio e perciò possono rappresentare «solo un unico momento dell'azione».

Mostriamo come il linguaggio figurativo non dia istantaneamente il senso di una immagine, ma implichi gli stessi tempi di elaborazione del linguaggio letterario e musicale.

Negli anni 1950-1960 il fisiologo russo Alfred Yarbus dimostrò sperimentalmente che la visione è un processo attivo costituito da molti movimenti oculari (per ogni secondo). Durante la visione vengono selezionate e ripetutamente scansionate solo alcune parti dell'immagine: quelle che presentano il maggior contenuto informativo.

In figura C28 (Yarbus, 1967), si vede la foto del busto della regina egiziana Nefertiti e il tracciato delle saccadi (moti oculari) e dei punti di fissazione rilevati su un osservatore. La distribuzione spaziale dei punti di fissazione mostra che la maggior parte del tempo è dedicata a guardare occhi, naso, bocca e orecchio, mentre gli altri elementi del volto, quali guancia e collo, vengono invece visualizzati per un tempo inferiore. Il confronto tra le due immagini mostra che il cervello umano ha bisogno di pochissimi punti per ricostruire l'immagine completa basandosi soprattutto sulle esperienze passate del soggetto e sulle sue aspettative. Nel 1967 non erano disponibili misuratori dei tempi che intercorrono fra due fissazioni successive; oggi questo è possibile con gli eyetracker (Fig. C29) e si verifica che l'elaborazione figurativa richiede gli stessi tempi delle altre elaborazioni linguistiche, con buona pace di Lessing.

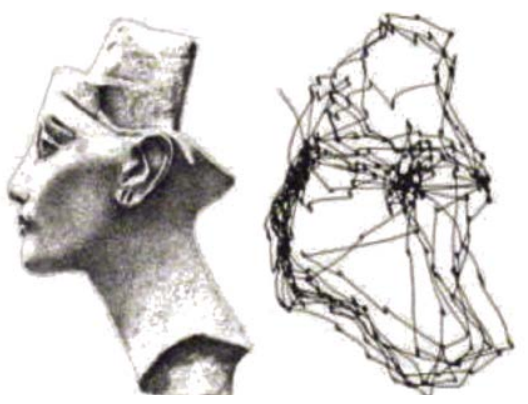

Figura C28 - Le registrazioni effettuate su un soggetto che guarda il busto della regina Nefertiti mettono in evidenza delle regolarità nei movimenti oculari. A sinistra, ciò che il soggetto vedeva; a destra, i suoi movimenti oculari registrati da Alfred L. Yarbus dell'Istituto per i problemi della trasmissione dell'informazione di Mosca. Gli occhi sembrano posarsi sui tratti della testa seguendo un tracciato abbastanza regolare piuttosto che casuale. 

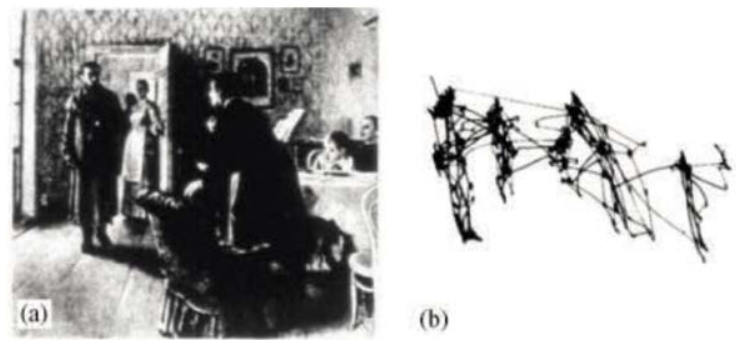

(b)
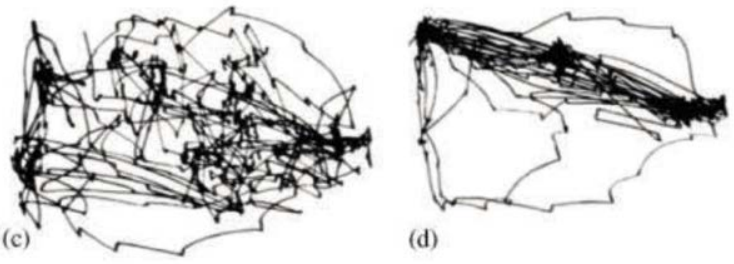

(d)

Figura C29 - Registrazioni fatte da Yarbus (1967) dei moti oculari di un soggetto che vede il quadro a) (Non lo aspettavano di I.P. Repin), con differenti obiettivi in mente: b) 'Ricordare i vestiti indossati dalla gente'; c) 'Ricordare le posizioni della gente e gli oggetti nella stanza'; d) 'Stimare quanto a lungo il visitatore inaspettato era stato via'.

Fin qui abbiamo visto solo correlazioni spaziali fra le successive fissazioni dell'occhio del soggetto che vede l'immagine. Oggi, con gli eye tracker, è possibile anche registrare le separazioni temporali. Sul «Journal of Eye Movement Research» (2007), Privitera, Stark e Zangemeister (University of California, Berkeley) hanno registrato i moti oculari di un soggetto che guardava un quadro di Bonnard e hanno misurato i tempi che separano due fissazioni successive. Questi tempi sono dell'ordine di quelli attribuiti alle operazioni linguistiche nella figura 7 della Parte A.

\section{Neuroscienza}

Tempi di comunicazione fra due cellule (Fig. C30) nei casi di:

a) cellule immerse in un liquido inter-cellulare (diffusione);

b) cellule collegate dal flusso di un fluido (linfa o sangue);

c) cellule cerebrali (neuroni) collegate da segnali elettrici.

Nella figura C31, si presentano le aree cerebrali deputate a compiti specializzati.

L'assone è una linea elettrica che collega il nucleo di un neurone con le estremità dette sinapsi. Nell'assone si propaga un segnale elettrico fatto di impulsi (spike) di durata circa $1 \mathrm{msec}$. La figura C32 mostra che l'assone è rivestito da una guaina mielinica, interrotta ogni tanto (i cosiddetti nodi di Ranvier). Alle interruzioni, lo scambio di ioni $\mathrm{Na}$ e K con il liquido intercellulare fornisce l'energia necessaria per attivare la propagazione elettrica. 
a)<smiles>C1CCCCCC1</smiles><smiles>O</smiles>

b)

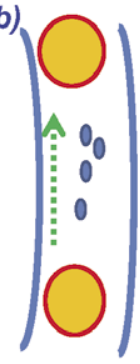

\section{Comunicazione fra due cellule}

a)Diffusione di molecole

(tempo cresce con quadrato distanza: $1 \mathrm{~ms}$ su

1 micron; oltre 1 ora per distanze $1 \mathrm{~mm}$ );

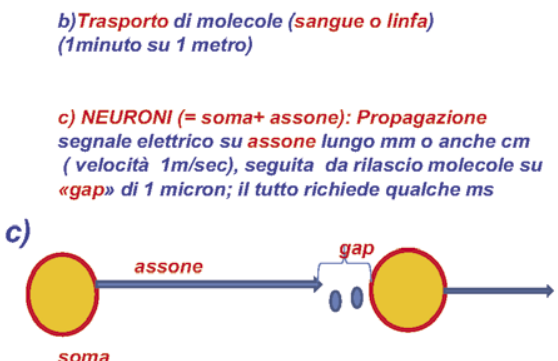

Figura C30 - La comunicazione fra due cellule può aver luogo per: a) diffusione di molecole nel liquido inter-cellulare; il tempo $\tau=1^{2} / D(1=$ separazione; $D=$ costante di diffusione $\approx 10^{-6} \mathrm{~cm}^{2} / \mathrm{sec}$ ) cresce con il quadrato di l; b) trasporto: $\tau=1 / \mathrm{v}(\mathrm{v}=$ velocità del fluido) cresce solo con 1 alla prima potenza (crescita lineare); velocità $\approx 1$ $\mathrm{m} /$ minuto; c) propagazione elettrica lungo l'assone del neurone; velocità $\approx 1 \mathrm{~m} / \mathrm{s}$ nel cervello, fino a $10 \mathrm{~m} / \mathrm{s}$ negli assoni che collegano il cervello agli arti.

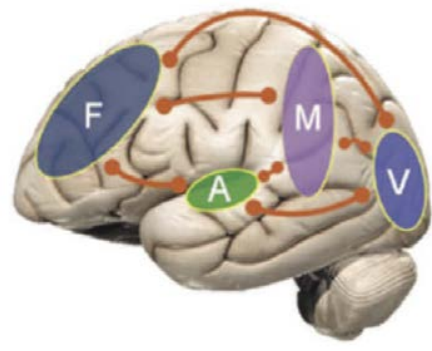

Figura C31 - Interazioni multisensoriali: si combinano in un segnale coordinato che coinvolge la corteccia frontale, le regioni temporo-parietali e le aree unimodali della corteccia: $\mathrm{A}=$ corteccia uditiva; $\mathrm{V}=$ corteccia visiva; $\mathrm{F}=$ corteccia pre-frontale; $\mathrm{M}=$ regioni multisensoriali . 
La sequenza di spikes è un codice che stimola all'estremità (sinapsi) l'emissione di trasmettitori chimici (neurotrasmettitori). Questi attraversano per diffusione il gap sinaptico (spazio intercellulare) e sono raccolti da un altro neutrone. Il percorso sull'assone è veloce (trasmissione elettrica); il percorso nel gap è anch'esso veloce; è invero diffusione, ma su distanza piccolissima (di qualche micrometro).

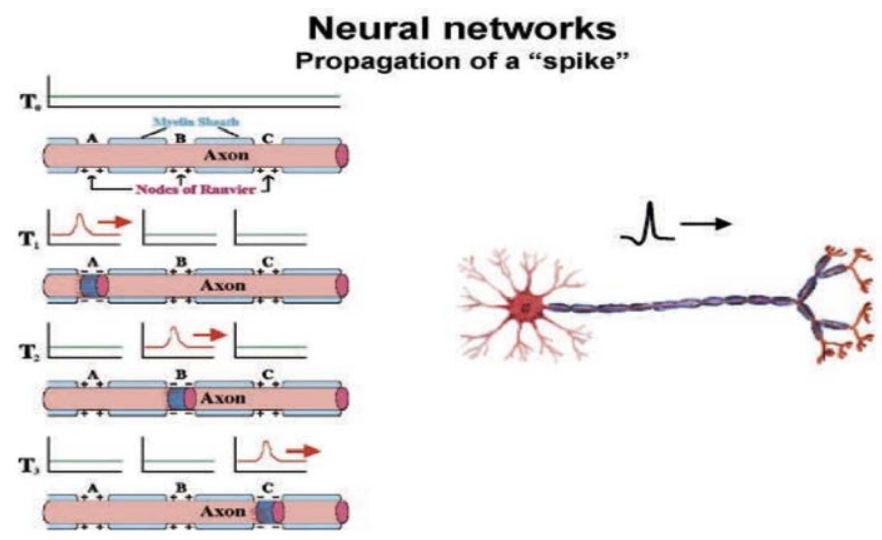

Figura C32 - Le cellule normali comunicano per segnali chimici, ma la diffusione è lenta perché il tempo si scala con il quadrato della distanza; invece l'assone dei neuroni è una linea di trasmissione elettrica dove il segnale viaggia ad $1 \mathrm{~m} / \mathrm{sec}$. Tempi di trasporto su una distanza $d=1 \mathrm{~mm}: 1$. diffusione $\left(d^{2}=D t\right)\left(D=10^{-6} \mathrm{~cm}^{2} / \mathrm{sec}\right) \rightarrow t=$ $10^{4} \mathrm{sec} ; 2$ propagazione elettrica $\left(v=10^{2} \mathrm{~cm} / \mathrm{sec}\right) t=\mathrm{d} / \mathrm{v}=1 \mathrm{msec}$.

Come già detto alla voce Consapevolezza PERCETtiva: codice neuronale $=$ treno spikes, ciascuna alta $100 \mathrm{mV}$, e di durata $1 \mathrm{~ms}$; con separazione temporale minima (bin) di $3 \mathrm{~ms}$ e separazione media (nella banda gamma dello EEG) di $20 \mathrm{~ms}$.

Comunicazione fra neuroni $=$ sincronizzazione (come una folla che batte le mani all'unisono): Fig. C33.

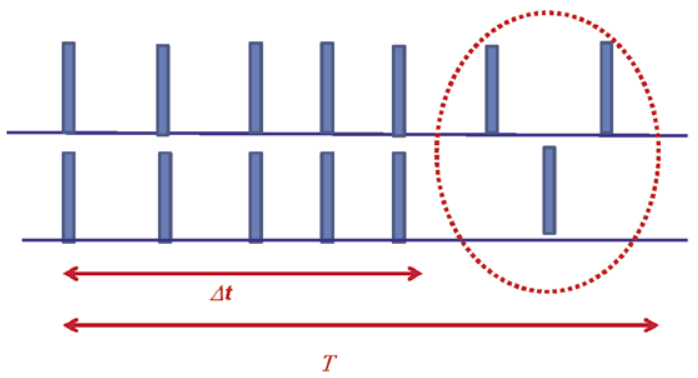

Figura C33 - Esempio di sincronizzazione fra due treni di spikes: efficace fino a $\mathrm{D} t$, ma non efficace su tutto l'intervallo temporale $T$. 
Nella figura 33 della Parte B abbiamo riportato un esperimento (del gruppo di W. Singer). Si trattava di feature binding, come sincronizzazione fra neuroni nel cui campo recettivo c'è la stessa figura: la donna e il gatto sono visti ciascuno da un insieme di neuroni. Questi insiemi racchiudono i neuroni che rivelano i dettagli specifici degli oggetti visuali 'neuroni' che hanno come oggetto lo stesso individuo; scaricano impulsi elettrici in sincronia, indipendentemente dal dettaglio che hanno nel proprio campo recettivo.

Il risultato della figura 33 è stato ottenuto inserendo micro elettrodi che sentono il singolo assone del cervello dell'animale di laboratorio esposto all'immagine.

Nel caso di soggetti umani, non potendo applicare oggetti invasivi, si ricorre allo EEG (elettro-encefalo-gramma) che si registra applicando al cranio del soggetto un casco che contiene molti elettrodi. Il campo elettrico di ogni assone si sovrappone ai campi di migliaia di altri assoni e si registra la somma. Dunque, l'EEG è povero come risoluzione spaziale, ma temporalmente può vedere i millisecondi.

Nel cervello, aree remote sono coordinate (cioè si sincronizzano) non per segnali diretti che si propagano su assoni (ci vorrebbe troppo tempo: se due aree distano di $10 \mathrm{~cm}$, i segnali assonali arriverebbero dopo $0,1 \mathrm{sec}$, invece la sincronizzazione assicura coincidenze entro qualche millisecondo), ma perché sentono il campo elettrico somma (Fig. C34).
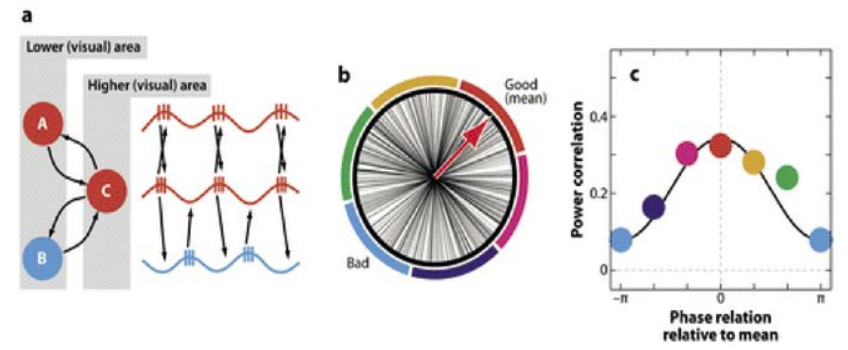

Figura C34 - Sincronizzazione fra aree visive distanti. A e C sono in fase, mentre B e $\mathrm{C}$ sono in controfase. Come avviene? Lo spiking ha luogo in presenza di un segnale di fondo alto, mentre è impedito da un segnale di fondo basso (spiegazione: vedi la figura 34 della Parte B, quando si è introdotto il caos omoclinico). Qui la coerenza di fase è assicurata dalla somma di tutti i segnali di unarea (ad esempio A) che può contenere milioni di neuroni. Dunque - come nello EEG - quello che conta è il segnale somma e non quello del singolo neurone. [Da: Fries, 2009]

\section{Probabilità, probabilità condizionata, teorema di Bayes}

La probabilità $P$ (discussa nella Parte $\mathrm{B}$ ) è un numero compreso fra 0 (assoluta incertezza) e 1 (assoluta certezza), come si può vedere dalla figura C35:

$$
0<P<1
$$




\section{Che cosa è la probabilità}
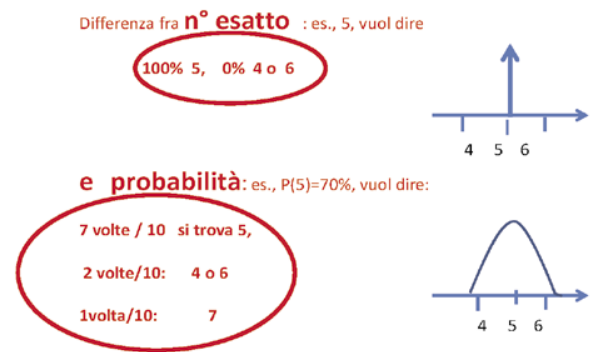

Prob. condizionata : $\mathrm{P}(\mathrm{A} \mid \mathrm{B})=$ se è accaduto $\mathrm{B}$,

è probabile (NON CERTO!) che poi si trovi $A$

Figura C35 - Differenza fra certezza e probabilità in una misura.

Per esempio, lancio di un dado a 6 facce: probabilità di un numero $=1 / 6$. Dati due gruppi di eventi che chiameremo $h=$ ipotesi e $d=$ dati, la probabilità congiunta è il prodotto

$$
P(h, d)=P(h) \cdot P(d)
$$

se gli eventi sono indipendenti; se invece l'uno dipende dall'altro (diremo «è condizionato»), allora scriveremo:

$$
P(h, d)=P(h) \cdot P(d \mid h)=P(d) \cdot P(h \mid d)
$$

Al riguardo, nelle figure $\mathrm{C} 35$ e C36 presentiamo ulteriori elementi utili, spiegati nelle didascalie.

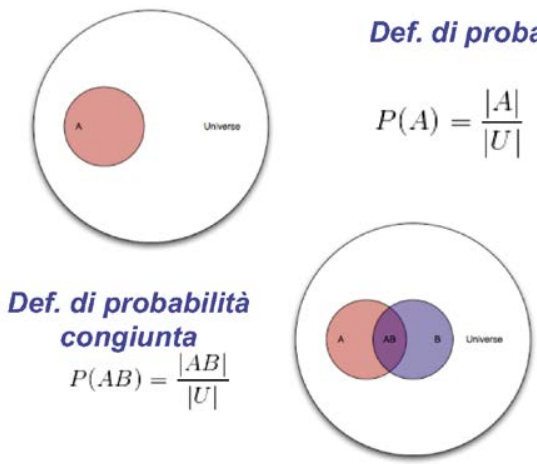

Figura C36 - Diagrammi di Venn che rendono intuitivi i concetti di probabilità: se il tutto è rappresentato dall'area di un cerchio, $P(A)$ è dato da un rapporto di aree. Lo stesso vale per la probabilità congiunta di due eventi $\mathrm{A}$ e $\mathrm{B}$, che è la sovrapposizione delle aree corrispondenti. 
Def. di probabilità condizionata

$$
P(A \mid B)=\frac{P(A B)}{P(B)} \quad P(B \mid A)=\frac{P(A B)}{P(A)}
$$

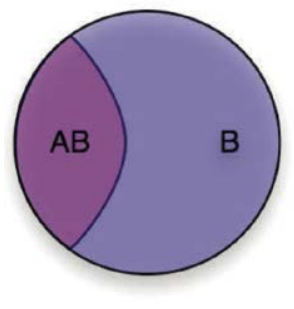

$$
P(A \mid B) P(B)=P(B \mid A) P(A)
$$

Questa relazione è il Teorema

di Bayes

$P(A \mid B)=\frac{P(B \mid A) P(A)}{P(B)}$

Figura C37 - Probabilità condizionata (= probabilità congiunta, divisa per la probabilità dell'evento condizionante): teorema di Bayes.

\section{Scala}

La nozione di scala corrisponde a una curva di probabilità che è stretta rispetto al valor medio (Fig. C38).

\section{La nozione di scala}
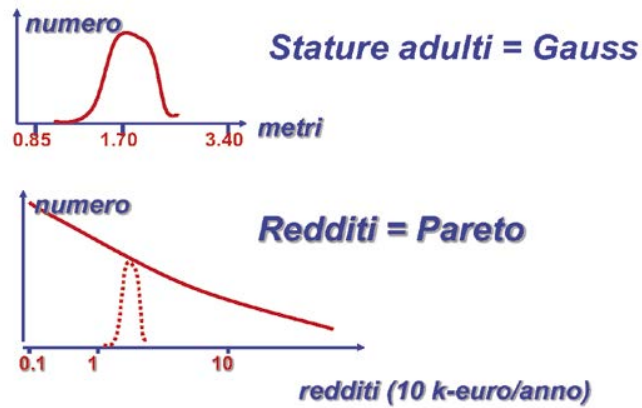

Figura C38 - La nozione di scala: per esempio la statura degli umani adulti ha una scala precisa, con uno sparpagliamento attorno alla media dato dalla curva a campana di Gauss. La distribuzione dei redditi (Pareto) è invece una curva senza una scala, e la possiamo vedere come la composizione di molte Gaussiane.

\section{Tempo, irreversibilità}

Nel par. 10 della Parte B abbiamo visto che spazio e tempo, sia in Newton che in Einstein, sono trattati nello stesso modo: si può andare concettual- 
mente avanti o indietro su un asse (retta per Newton, curva per Einstein). Sappiamo dall'esperienza di tutti i giorni che spazialmente possiamo andare a destra o sinistra, ma temporalmente non possiamo tornare indietro.

Qualunque oggetto macroscopico obbedisce al II principio della termodinamica, cioè il tempo fluisce solo nella direzione per cui cresce il disordine, cioè aumenta l'entropia (Fig. C39).

\title{
Da una a molte particelle: \\ termodinamica, entropia, freccia del tempo
}

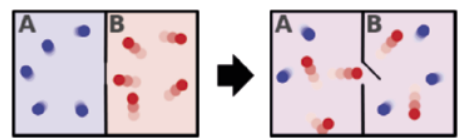

sistema (costituito da due gas differenti) in cui si ha aumento di entropia

\begin{abstract}
Entropia $=\mathrm{n}$. di modi diversi di ri-arrangiare le parti più piccole del sistema arrivando sempre allo stesso sistema globale: cresce all'aumentare del tempo.

Nella teoria dell'informazione l'entropia misura l'incertezza presente in un segnale aleatorio.
\end{abstract}

Figura C39 - Se mettiamo in comunicazione due celle che contengono gas diversi, dopo un po' si ha un rimescolamento; le particelle si re-distribuiscono in modo uniforme fra le due celle. Non avviene mai il contrario (vedi par. 11 della Parte B). 


\section{NOTA BIBLIOGRAFICA}

\section{CONTRIBUti DELl'AUtORE AGLI ARgOMENTI DEL VOLUME}

L'elenco seguente riassume i contributi dell'autore relativi agli argomenti trattati nel presente libro'.

(1965) Coerenza della luce laser misurata mediante la statistica dei fotoni.

(1982) Caos deterministico (geometrico) nei laser.

(1987) Caos omoclinico nei laser.

(2001) Sincronizzazione del caos omoclinico.

(2004) Caos omoclinico e sincronizzazione applicati ai processi cerebrali.

(2007) Bayes diretto nelle percezioni.

(2009) Bayes inverso, nelle elaborazioni linguistiche.

Un'introduzione a questi argomenti è stata presentata in:

(2012) Fenomenologia della coscienza: dall'apprensione al giudizio, in A. Ales Bello, P. Manganaro (a cura di), ... e la coscienza? Fenomenologia, psicopatologia, neuroscienze, Laterza, Roma-Bari, pp. 841-887.

Su quanto trattato nelle Parti B e C:

(1990), con Arecchi I., I simboli e la realtà, JacaBook, Milano (note di un

Corso per l'Istituto Teologico dell'Italia Centrale).

(2004) Caos e complessità nel vivente, IUSS Press, Pavia (lezioni tenute alla Scuola Universitaria Superiore di Pavia).

(2007) Coerenza, Complessità, Creatività, Di Renzo, Roma

SELEZIONE DI CONTRIBUTI FONDAMENTALI PER QUANTO TRATTATO IN QUESTO LIBRO

Bateson G. (1977), Verso un'ecologia della mente (ed. orig. 1972), Adelphi, Milano.

Fries P. (2009), Neuronal gamma-band synchronization as a fundamental process in cortical computation, "Annu Rev Neurosci.», 32, pp. 209224., doi: 10.1146/annurev.neuro.051508.135603.

1 Per l'intera bibliografia e la descrizione della mia vita scientifica si veda l'indirizzo web <http://www.ino.it/home/arecchi/>. 
Frye N. (1986), Il grande codice. La Bibbia e la letteratura (ed. orig. 1981), Einaudi, Torino.

Kuhn T. (1999), La struttura delle rivoluzioni scientifiche (ed. orig. 1962), Einaudi, Torino.

Livi A. (1990), Filosofia del senso comune. Logica della scienza e della fede, Edizioni Ares, Milano.

Lonergan B. (2007), Insight. Uno studio del comprendere umano (ed. orig. 1957), Città nuova, Roma.

von Humboldt W. (2004), La diversità delle lingue (ed. orig. 1836), Laterza, Roma-Bari.

Yarbus A. (1967), Eye movements and vision, Plenum Press, New York.

\section{LAVORI CRUCIALI PER GLI ARGOMENTI TRATTATI IN QUESTO LIBRO}

Applicazioni di caos omoclinico e sincronizzazione, dai laser ai processi cerebrali

Arecchi F.T. (2004), Chaotic neuron dynamics, synchronization and feature binding, «Physica», A 338, p. 218.

ART (Adaptive Resonance Theory)

Carpenter G.A., Grossberg S. (2003), Adaptive Resonance Theory, in M.A. Arbib (ed.), The Handbook of Brain Theory and NeuralNetworks (2nd Edition), MIT Press, Cambridge, MA.

Bayes diretto, nelle percezioni

Arecchi (2007), Physics of cognition: complexity and creativity, «Eur. Phys. J. Special Topics», 146, p. 205.

Doya K., Ishii S., Pouget A. (eds.) (2007), Bayesian Brain: Probabilistic Approaches to Neural Codingi, MIT Press, Cambridge, MA.

Kording K.P., Wolpert, D.M. (2008), Bayesian decision theory in sensorymotor control, «Trends in Cognitive Sciences», 10, p. 319.

Bayes inverso, nelle elaborazioni linguistiche)

Arecchi F.T. (2010), Dynamics of consciousness: complexity and creativity, «The Journal of Psychophysiology», XXIV (2), pp. 141-148.

Complessità algoritmica

Chaitin G.J. (1987), Algorithmic information theory, Cambridge University Press.

\section{Feature binding}

Rodriguez E., George N., Lachaux J-P., Martinerie J., Renault B., Varela

F.J. (1999), Perception's shadow: long distance synchronization of human brain activity, «Nature», 397, pp. 430-433.

SingerW. (2007), Binding by synchrony, «Scholarpedia», II (12), p. 1657. 
Fisica e conoscenza

Ellis G., Silk J. (2014), Defend the integrity of physics, «Nature», 516, 121.

Fisiologia e conoscenza

Hubel D.H. (1989), Occhio, Cervello, Visione (titolo orig. Eye, Brain and Vision), Zanichelli, Bologna.

Singer W., Gray C.M. (1995), Visual Feature Integration and the Temporal Correlation Hypothesis, "Annual Review of Neuroscience», XVIII, pp. 555-558.

Intelligenza artificiale

Turing A. (1950), Computing machinery and intelligence, «Mind», LIX, pp. 433-460.

Global Work Space (GWS)

Dehaene S., Naccache L. (2001), Towards a cognitive neuroscience of consciousness: Basic evidence and a workspace framework, "Cognition», 79, pp. 1-37.

Inferenza di Bayes

Bayes Th. (1763), An Essay toward solving a Problem in the Doctrine of Chances, «Philosophical Transactions of the Royal Society of London", 53, pp. 370-418.

Processi linguistici, finestra di $3 \mathrm{sec}$

Pöppel E. (2004), Lost in time: a historical frame, elementary processing units and the 3-second window, "Acta Neurobiologiae Experimentalis", 64, pp. 295-301.

Arecchi F.T., Farini A., Megna N. (2016), A test of multiple correlation temporal window characteristic of non-Markov processes, "European Phys. J. Plus», 131, p. 50. 

abilità linguistica 5

AI vedi intelligenza artificiale

algoritmo/i 6, 91-92

- genetico/i 13, 91

analogia $32-33$

apprensione 10, 13

ART (Adaptive Resonance Theory) $49,92-95$

assoni 85

atomo/i 60

— di Bohr 69-71

BACON 27

Barlow W. 88

bastoncelli 84

Bayes

- diretto 7, 17,97

- inverso 7, 16-17, 96-98

bit 13

bottom-up 98

bursting 102-103

campo/i

- di forza 61

- elettrico 58

caos, 27

- controllo del 24, 27, 103

- deterministico 46-50, 99-103

- geometrico 99

- omoclinico 100-102

- temporale 99-100

catastrofi 32

cellule gangliari 87

cinematica 52

codice
- cerebrale 103-106

- temporale 105

coerenza 28

cognitivismo 3

cognizione 5

colle di probabilità 13-15

- multipli 14

complessità 106-107

- algoritmica 13, 44-45, 107

- e complicazione 108

- epistemica 32

- formale 44

- ontica 32

- semantica 13-15, 28, 45-46, 107-108

comportamentismo 3

computazione quantistica 112

coni 84

consapevolezza percettiva 7,12

- e NCC 108-110

contingenza 33

coscienza 12

- di se stessi, 15, 18

coulomb (C) 58

corpi genicolati laterali 87

corteccia pre-frontale vedi PFC

creatività 28

decoerenza 112

delta di Dirac 22, 110-111

densità 67

determinismo 59

dialogo inter-personale 5

diffrazione 62

dimensioni frattali 111 
dinamica 52

- ed energia 52-58

- lineare 54

- non lineare 56

- omoclinica 48

disordine 77

effetto

- farfalla 99

- fotoelettrico 68-69

elettrone 60

energia 55

- cinetica 55

- e dinamica $52-58$

- e vita 64-65

- potenziale 55

entanglement 112

entropia 77, 128

- delle percezioni 105-106

- di Kolmogorov 24

- e macchine termiche 76-78

epicicli di Tolomeo 28

epigenetica 27, 112

equazioni di Maxwell 15

equilibrio 112

ermeneutica

- a circolo 5, 28-32

- a spira 5, 28-32

eyetracker 121

feature binding 47-48

- e codice temporale 87-89

feedback 47

- meccanismo di 94-95

fisica

- del disordine 76-83

- delle percezioni 83-87

- e fisiologia 50-52

- programma della 41-44

- quantistica 71-72

fissione vedi scissione

forze

- di gravitazione 60

- elettriche 60

- lineari 113-114

- non lineari 113-114
- nucleari

fotoni vedi quanti

funzione di Wigner 103-105

fusione 60

Galileo Galilei 114-115

gene 115

- esone 115

- introne 115

giudizio $6-7,12-13$

grammo-molecola 59

grandezze specifiche 58

gruppo di ri-normalizzazione di Wilson 25

GWS (global work space) 8, 116-117

HC (Homoclinic Chaos) vedi dinamica omoclinica

impulso 69

inferenza di Bayes 7, 96-98 vedi anche Apprensione

- e percezione 7-12

informazione $82-83$

intelligenza artificiale (AI) 34

- debole (weak AI) 35

- e machine learning 34-37

- forte (strong AI) 35

interferenza

- costruttiva 62

- distruttiva 62

- parziale 62

ippocampo 120

joule 57

lavoro 54

linguaggio

- e programmi scientifici $26-28$

- nascita del 15-20

- teoria matematica del 37

- senso comune di 38-39

macchina di Turing 118-119

machine learning 34

- e intelligenza artificiale 34-37 
matematizzazione, limiti della 23 meccanica quantistica 71

- difficoltà della 72-73

meccanicismo 52

meme 119

memoria 119-120

- a breve termine 7-8, 93-94, 120

- a lungo termine 7, 93-94, 120

Mendeelev D.I 25

- tavola di 25

mito platonico della caverna 8 , 16

mole vedi grammo-molecola

molecole 59

moti oculari 121-122

multiversi 28

mutazione 11

NCC (neural correlates of consciousness) 13

- e consapevolezza percettiva $108-110$

neuro-derivati 19

neuroscienza $122-125$

neurone/i

- e laser 100-101

- specchio 18

neutroni 60

newton $(\mathrm{N}) 58$

nucleoni 60

onde 61-63

- gravitazionali 73-76

- interferenza delle 62

- longitudinali 62

- trasversali 62

PACknowledge (conoscenza «probably approximately correct») 11 percezione/i 1, 6, 12-13

- come inferenza di Bayes 7-12

PFC (pre-frontal cortex) 8

potenziale elettrico 58

principio

- della termodinamica, secondo 77
- di complementarietà 72

- di esclusione di Pauli 25

- di indeterminazione di Heisenberg 43,73

- di ragione non sufficiente 79 probabilità 78-81, 125-127

- concetto frequentistico di 79-80

- concetto logistico di 80

- condizionata 126

- congiunta 126

- soggettiva 80-81

procedura

- algoritmica 15

- linguistica 12

processo visivo, stadi del $85-87$

protone 60

quanti $64,66-68$

quarks 61

radioattività 60

reazioni esotermiche 66

relatività

— galileiana 73

- generale 74

- ristretta 73

relazione di indeterminazione di Heisenberg 43

rivoluzione umana 18

ri-codifica 27

riduzionismo 50,59

salto

- di paradigma 11, 14, 26, 98

- non algoritmico 14-15

scala/e 127

- gerarchia di 116

scienza normale $11,14,98$

scissione 60

selezione 11

semiosi 27

sistemi

- aperti 32

- chiusi 32

spettro

- di corpo nero 67 
- elettromagnetico 63 spikes 2,47

spostamento 55

stanza cinese 36

tempo 127-128

teorema di Goedel 14

teoria

- dell'informazione 128

- efficace 27,32 termodinamica 76

- II principio della 77

test di Turing 36

top-down 98

unità di misura 58

vettori 53

wormholes 74 


\section{STUDI E SAGGI}

Titoli Pubblicati

\section{ARCHITETTURA, STORIA DELL'ARTE E ARCHEOLOGIA}

Acciai S., Sedad Hakki Eldem. An aristocratic architect and more

Bartoli M.T., Lusoli M. (a cura di), Le teorie, le tecniche, i repertori figurativi nella prospettiva d'architettura tra il '400 e il '700. Dall'acquisizione alla lettura del dato

Bartoli M.T., Lusoli M. (a cura di), Diminuzioni e accrescimenti. Le misure dei maestri di prospettiva

Benelli E., Archetipi e citazioni nel fashion design

Benzi S., Bertuzzi L., Il Palagio di Parte Guelfa a Firenze. Documenti, immagini e percorsi multimediali

Biagini C. (a cura di), L’Ospedale degli Infermi di Faenza. Studi per una lettura tipomorfologica dell'edilizia ospedaliera storica

Bologna A., Pier Luigi Nervi negli Stati Uniti 1952-1979. Master Builder of the Modern Age

Eccheli M.G., Pireddu A. (a cura di), Oltre l'Apocalisse. Arte, Architettura, Abbandono

Fischer von Erlach J.B., Progetto di unarchitettura istorica / Entwurff einer Historischen Architectur, traduzione e cura di G. Rakowitz

Frati M., "De bonis lapidibus conciis": la costruzione di Firenze ai tempi di Arnolfo di Cambio. Strumenti, tecniche e maestranze nei cantieri fra XIII e XIV secolo

Gregotti V., Una lezione di architettura. Rappresentazione, globalizzazione, interdisciplinarità

Gulli R., Figure. Ars e ratio nel progetto di architettura

Lisini C., Lezione di sguardi. Edoardo Detti fotografo

Maggiora G., Sulla retorica dell'architettura

Mantese E. (a cura di), House and Site. Rudofsky, Lewerentz, Zanuso, Sert, Rainer

Mazza B., Le Corbusier e la fotografia. La vérité blanche

Mazzoni S. (a cura di), Studi di Archeologia del Vicino Oriente. Scritti degli allievi fiorentini per Paolo Emilio Pecorella

Messina M.G., Paul Gauguin. Un esotismo controverso

Pireddu A., In abstracto. Sull'architettura di Giuseppe Terragni

Pireddu A., The Solitude of Places. Journeys and Architecture on the Edges

Pireddu A., In limine. Between Earth and Architecture

Rakowitz G., Tradizione Traduzione Tradimento in Johann Bernhard Fischer von Erlach

Tonelli M.C., Industrial design: latitudine e longitudine

\section{CULTURAL STUDIES}

Candotti M.P., Interprétations du discours métalinguistique. La fortune du sūtra A 1.1.68 chez Patañjali et Bhartrhari

Nesti A., Per una mappa delle religioni mondiali

Nesti A., Qual è la religione degli italiani? Religioni civili, mondo cattolico, ateismo devoto, fede, laicità

Pedone V., A Journey to the West. Observations on the Chinese Migration to Italy

Pedone V., Sagiyama I. (edited by), Perspectives on East Asia

Pedone V., Sagiyama I. (edited by), Transcending Borders. Selected papers in East Asian studies

Rigopoulos A., The Mahānubhāvs

Squarcini F. (a cura di), Boundaries, Dynamics and Construction of Traditions in South Asia

Vanoli A., Il mondo musulmano e i volti della guerra. Conflitti, politica e comunicazione nella storia dell'islam 


\section{DIRITTO}

Allegretti U., Democrazia partecipativa. Esperienze e prospettive in Italia e in Europa

Bartolini A., Pioggia A. (a cura di), A 150 anni dall'unificazione amministrativa italiana. Studi. Vol. VIII. Cittadinanze amministrative

Cafagno M., Manganaro F. (a cura di), A 150 anni dall'unificazione amministrativa italiana. Studi. Vol. V. L'intervento pubblico nell'economia

Cavallo Perin R., Police A., Saitta F. (a cura di), A 150 anni dall'unificazione amministrativa italiana. Studi. Vol. I. L'organizzazione delle pubbliche amministrazioni tra Stato nazionale e integrazione europea

Chiti E., Gardini G., Sandulli A. (a cura di), A 150 anni dall'unificazione amministrativa italiana. Studi. Vol. VI. Unità e pluralismo culturale

Cingari F. (a cura di), Corruzione: strategie di contrasto (legge 190/2012)

Civitarese Matteucci S., Torchia L., A 150 anni dall'unificazione amministrativa italiana. Studi. Vol. IV. La tecnificazione

Comporti G.D. (a cura di), A 150 anni dall'unificazione amministrativa italiana. Studi. Vol. VII. La giustizia amministrativa come servizio (tra effettività ed efficienza)

Curreri S., Democrazia e rappresentanza politica. Dal divieto di mandato al mandato di partito

Curreri S., Partiti e gruppi parlamentari nell'ordinamento spagnolo

De Giorgi Cezzi, Portaluri Pier Luigi (a cura di), A 150 anni dall'unificazione amministrativa italiana. Studi. Vol. II. La coesione politico-territoriale

Federico V., Fusaro C. (a cura di), Constitutionalism and Democratic Transitions. Lessons from South Africa

Fiorita N., L'Islam spiegato ai miei studenti. Otto lezioni su Islam e diritto

Fiorita N., L'Islam spiegato ai miei studenti. Undici lezioni sul diritto islamico

Fossum J.E., Menéndez A.J., La peculiare costituzione dell'Unione Europea

Gregorio M., Le dottrine costituzionali del partito politico. L'Italia liberale

Marchetti B., Renna M. (a cura di), A 150 anni dall'unificazione amministrativa italiana. Studi. Vol. III. La giuridificazione

Palazzo F., Bartoli R. (a cura di), La mediazione penale nel diritto italiano e internazionale

Ragno F., Il rispetto del principio di pari opportunità. L'annullamento della composizione delle giunte regionali e degli enti locali

Sorace D. (a cura di), Discipine processuali differenziate nei diritti amministrativi europei

Trocker N., De Luca A. (a cura di), La mediazione civile alla luce della direttiva 2008/52/CE

Urso E., La mediazione familiare. Modelli, principi, obiettivi

Urso E., Le ragioni degli altri. Mediazione e famiglia tra conflitto e dialogo. Una prospettiva comparatistica e interdisciplinare

\section{ECONOMIA}

Bardazzi R. (edited by), Economic multisectoral modelling between past and future. A tribute to Maurizio Grassini and a selection of his writings

Bardazzi R., Ghezzi L. (edited by), Macroeconomic modelling for policy analysis

Barucci P., Bini P., Conigliello L. (a cura di), Economia e Diritto durante il Fascismo. Approfondimenti, biografie, nuovi percorsi di ricerca

Ciampi F., Come la consulenza direzionale crea conoscenza. Prospettive di convergenza tra scienza e consulenza

Ciampi F., Knowing Through Consulting in Action. Meta-consulting Knowledge Creation Pathways

Ciappei C. (a cura di), La valorizzazione economica delle tipicità rurali tra localismo e globalizzazione

Ciappei C., Citti P., Bacci N., Campatelli G., La metodologia Sei Sigma nei servizi. Un'applicazione ai modelli di gestione finanziaria

Ciappei C., Sani A., Strategie di internazionalizzazione e grande distribuzione nel settore dell'abbigliamento. Focus sulla realtà fiorentina

Garofalo G. (a cura di), Capitalismo distrettuale, localismi d'impresa, globalizzazione 
Laureti T., L'efficienza rispetto alla frontiera delle possibilità produttive. Modelli teorici ed analisi empiriche

Lazzeretti L. (a cura di), Art Cities, Cultural Districts and Museums. An Economic and Managerial Study of the Culture Sector in Florence

Lazzeretti L. (a cura di), I sistemi museali in Toscana. Primi risultati di una ricerca sul campo

Lazzeretti L., Cinti T., La valorizzazione economica del patrimonio artistico delle città d'arte. Il restauro artistico a Firenze

Lazzeretti L., Nascita ed evoluzione del distretto orafo di Arezzo, 1947-2001. Primo studio in una prospettiva ecology based

Meade S. Douglas (edited by), In Quest of the Craft. Economic Modeling for the 21st Century

Simoni C., Approccio strategico alla produzione. Oltre la produzione snella

Simoni C., Mastering the Dynamics of Apparel Innovation

FILOSOFIA

Baldi M., Desideri F. (a cura di), Paul Celan. La poesia come frontiera filosofica

Barale A., La malinconia dell'immagine. Rappresentazione e significato in Walter Benjamin e Aby Warburg

Berni S., Fadini U., Linee di fuga. Nietzsche, Foucault, Deleuze

Borsari A., Schopenhauer educatore? Storia e crisi di un'idea tra filosofia morale, estetica e antropologia

Brunkhorst H., Habermas

Cambi F., Pensiero e tempo. Ricerche sullo storicismo critico: figure, modelli, attualità

Cambi F., Mari G. (a cura di), Giulio Preti: intellettuale critico e filosofo attuale

Casalini B., Cini L., Giustizia, uguaglianza e differenza. Una guida alla lettura della filosofia politica contemporanea

Desideri F., Matteucci G. (a cura di), Dall'oggetto estetico all'oggetto artistico

Desideri F., Matteucci G. (a cura di), Estetiche della percezione

Di Stasio M., Alvin Plantinga: conoscenza religiosa e naturalizzazione epistemologica

Giovagnoli R., Autonomy: a Matter of Content

Honneth A., Capitalismo e riconoscimento

Mindus P., Cittadini e no: Forme e funzioni dell'inclusione e dell'esclusione

Sandrini M.G.,LafilosofiadiR. Carnaptraempirismoetrascendentalismo. (Inappendice: $R$. Carnap Sugli enunciati protocollari, Traduzione e commento di E. Palombi)

Solinas M., Psiche: Platone e Freud. Desiderio, sogno, mania, eros

Trentin B., La Città del lavoro. Sinistra e crisi del fordismo, a cura di Iginio Ariemma

Valle G., La vita individuale. L'estetica sociologica di Georg Simmel

FISICA

Arecchi F.T., Cognizione e realtà

LETTERATURA, FILOLOGIA E LINGUISTICA

Bastianini G., Lapini W., Tulli M., Harmonia. Scritti di filologia classica in onore di Angelo Casanova

Bilenchi R., The Conservatory of Santa Teresa

Bresciani Califano M., Piccole zone di simmetria. Scrittori del Novecento

Caracchini C., Minardi E. (a cura di), Il pensiero della poesia. Da Leopardi ai contemporanei. Letture dal mondo di poeti italiani

Cauchi-Santoro R., Beyond the Suffering of Being: Desire in Giacomo Leopardi and Samuel Beckett

Colucci D., L'Eleganza è frigida $e$ L'Empire des signs. Un sogno fatto in Giappone

Dei L. (a cura di), Voci dal mondo per Primo Levi. In memoria, per la memoria

Ferrone S., Visioni critiche. Recensioni teatrali da «l'Unità-Toscana» (1975-1983), a cura di Teresa Megale e Francesca Simoncini 
Ferrara M.E., Il realismo teatrale nella narrativa del Novecento: Vittorini, Pasolini, Calvino Filipa L.V., Altri orientalismi. L'India a Firenze 1860-1900

Francese J., Leonardo Sciascia e la funzione sociale degli intellettuali

Francese J., Vincenzo Consolo: gli anni de «l'Unità» (1992-2012), ovvero la poetica della colpa-espiazione

Franchini S., Diventare grandi con il «Pioniere» (1950-1962). Politica, progetti di vita e identità di genere nella piccola posta di un giornalino di sinistra

Francovich Onesti N., I nomi degli Ostrogoti

Frau O., Gragnani C., Sottoboschi letterari. Sei case studies fra Otto e Novecento. Mara Antelling, Emma Boghen Conigliani, Evelyn, Anna Franchi, Jolanda, Flavia Steno

Frosini G., Zamponi S. (a cura di), Intorno a Boccaccio / Boccaccio e dintorni

Galigani G., Salomè, mostruosa fanciulla

Gori B., La grammatica dei clitici portoghesi. Aspetti sincronici e diacronici

Gorman M., I nostri valori, rivisti. La biblioteconomia in trasformazione

Graziani M., Abbati O., Gori B. (a cura di), La spugna è la mia anima. Omaggio a Piero Ceccucci

Graziani M. (a cura di), Un incontro lusofono plurale di lingue, letterature, storie, culture

Guerrini M., De bibliothecariis. Persone, idee, linguaggi

Guerrini M., Mari G. (a cura di), Via verde e via d'oro. Le politiche open access dell'Università di Firenze

Keidan A., Alfieri L. (a cura di), Deissi, riferimento, metafora

Lopez Cruz H., America Latina aportes lexicos al italiano contemporaneo

Mario A., Italo Calvino. Quale autore laggiù attende la fine?

Masciandaro F., The Stranger as Friend: The Poetics of Friendship in Homer, Dante, and Boccaccio

Nosilia V., Prandoni M. (a cura di), Trame controluce. Il patriarca 'protestante' Cirillo Loukaris / Backlighting Plots. The 'Protestant' Patriarch Cyril Loukaris

Pestelli C., Carlo Antici e l'ideologia della Restaurazione in Italia

Rosengarten F., Through Partisan Eyes.. My Friendships, Literary Education, and Political Encounters in Italy (1956-2013). With Sidelights on My Experiences in the United States, France, and the Soviet Union

Ross S., Honess C. (edited by), Identity and Conflict in Tuscany

Totaro L., Ragioni d'amore. Le donne nel Decameron

Turbanti S., Bibliometria e scienze del libro: internazionalizzazione e vitalità degli studi italiani

Virga A., Subalternità siciliana nella scrittura di Luigi Capuana e Giovanni Verga

Zamponi S. (a cura di), Intorno a Boccaccio / Boccaccio e dintorni 2015

Zamponi S. (a cura di), Intorno a Boccaccio / Boccaccio e dintorni 2016

\section{MEDICINA}

Mannaioni P.F., Mannaioni G., Masini E. (a cura di), Club drugs. Cosa sono e cosa fanno Saint S., Krein S.L. (con Stock R.W.), La prevenzione delle infezioni correlate all'assistenza. Problemi reali, soluzioni pratiche

PEDAGOGIA

Mariani A. (a cura di), L'orientamento e la formazione degli insegnanti del futuro

POLITICA

Caruso S., Homo oeconomicus. Paradigma, critiche, revisioni

Cipriani A. (a cura di), Partecipazione creativa dei lavoratori nella 'fabbrica intelligente'. Atti del Seminario di Roma, 13 ottobre 2017

Cipriani A., Gramolati A., Mari G. (a cura di), Il lavoro 4.0. La Quarta Rivoluzione industriale e le trasformazioni delle attività lavorative

Corsi C. (a cura di), Felicità e benessere. Una ricognizione critica

Corsi C., Magnier A., L'Università allo specchio. Questioni e prospettive 
De Boni C., Descrivere il futuro. Scienza e utopia in Francia nell'età del positivismo

De Boni C. (a cura di), Lo stato sociale nel pensiero politico contemporaneo. 1. L'Ottocento

De Boni C., Lo stato sociale nel pensiero politico contemporaneo. Il Novecento. Parte prima: da inizio secolo alla seconda guerra mondiale

De Boni C. (a cura di), Lo stato sociale nel pensiero politico contemporaneo. Il Novecento. Parte seconda: dal dopoguerra a oggi

Gramolati A., Mari G. (a cura di), Bruno Trentin. Lavoro, libertà, conoscenza

Gramolati A., Mari G. (a cura di), Il lavoro dopo il Novecento: da produttori ad attori sociali. La Città del lavoro di Bruno Trentin per un' «altra sinistra»

Lombardi M., Fabbrica 4.0: i processi innovativi nel Multiverso fisico-digitale

Ricciuti R., Renda F., Tra economia e politica: l'internazionalizzazione di Finmeccanica, Eni ed Enel

Spini D., Fontanella M. (a cura di), Sognare la politica da Roosevelt a Obama. Il futuro dell'America nella comunicazione politica dei democrats

Tonini A., Simoni M. (a cura di), Realtà e memoria di una disfatta. Il Medio Oriente dopo la guerra dei Sei Giorni

Zolo D., Tramonto globale. La fame, il patibolo, la guerra

\section{PSICOLOGIA}

Aprile L. (a cura di), Psicologia dello sviluppo cognitivo-linguistico: tra teoria e intervento

Barni C., Galli G., La verifica di una psicoterapia cognitivo-costruttivista sui generis

Luccio R., Salvadori E., Bachmann C., La verifica della significatività dell'ipotesi nulla in psicologia

\section{SCIENZE NATURALI}

Bessi F.V., Clauser M., Le rose in fila. Rose selvatiche e coltivate: una storia che parte da lontano

Sánchez-Villagra M.R., Embrioni nel tempo profondo. Il registro paleontologico dell'evoluzione biologica

\section{SOCIOLOGIA}

Alacevich F., Promuovere il dialogo sociale. Le conseguenze dell'Europa sulla regolazione del lavoro

Alacevich F.; Bellini A., Tonarelli A., Una professione plurale. Il caso dell'avvocatura fiorentina

Battiston S., Mascitelli B., Il voto italiano all'estero. Riflessioni, esperienze e risultati di un'indagine in Australia

Becucci S., Garosi E., Corpi globali. La prostituzione in Italia

Bettin Lattes G., Giovani Jeunes Jovenes. Rapporto di ricerca sulle nuove generazioni e la politica nell'Europa del sud

Bettin Lattes G. (a cura di), Per leggere la società

Bettin Lattes G., Turi P. (a cura di), La sociologia di Luciano Cavalli

Burroni L., Piselli F., Ramella F., Trigilia C., Città metropolitane e politiche urbane

Catarsi E. (a cura di), Autobiografie scolastiche e scelta universitaria

Leonardi L. (a cura di), Opening the European Box. Towards a New Sociology of Europe

Nuvolati G., Mobilità quotidiana e complessità urbana

Nuvolati G., L'interpretazione dei luoghi. Flânerie come esperienza di vita

Nuvolati G., Sviluppo urbano e politiche per la qualità della vita

Ramella F., Trigilia C. (a cura di), Reti sociali e innovazione. I sistemi locali dell'informatica

Rondinone A., Donne mancanti. Un'analisi geografica del disequilibrio di genere in India

STORIA E SOCIOLOGIA DELLA SCIENZA

Angotti F., Pelosi G., Soldani S. (a cura di), Alle radici della moderna ingegneria. Competenze e opportunità nella Firenze dell'Ottocento

Cabras P.L., Chiti S., Lippi D. (a cura di), Joseph Guillaume Desmaisons Dupallans. La Francia alla ricerca del modello e l'Italia dei manicomi nel 1840 
Califano S., Schettino V., La nascita della meccanica quantistica

Cartocci A., La matematica degli Egizi. I papiri matematici del Medio Regno

Fontani M., Orna M.V., Costa M., Chimica e chimici a Firenze. Dall'ultimo dei Medici al Padre del Centro Europeo di Risonanze Magnetiche

Guatelli F. (a cura di), Scienza e opinione pubblica. Una relazione da ridefinire

Massai V., Angelo Gatti (1724-1798)

Meurig T.J., Michael Faraday. La storia romantica di un genio

Schettino V., Scienza e arte. Chimica, arti figurative e letteratura

STUDI DI BIOETICA

Baldini G. (a cura di), Persona e famiglia nell'era del biodiritto. Verso un diritto comune europeo per la bioetica

Baldini G., Soldano M. (a cura di), Nascere e morire: quando decido io? Italia ed Europa a confronto

Baldini G., Soldano M. (a cura di), Tecnologie riproduttive e tutela della persona. Verso un comune diritto europeo per la bioetica

Bucelli A. (a cura di), Produrre uomini. Procreazione assistita: un'indagine multidisciplinare

Costa G., Scelte procreative e responsabilità. Genetica, giustizia, obblighi verso le generazioni future

Galletti M., Zullo S. (a cura di), La vita prima della fine. Lo stato vegetativo tra etica, religione e diritto

STUDI EUROPEI

Guderzo M., Bosco A. (edited by), A Monetary Hope for Europe. The Euro and the Struggle for the Creation of a New Global Currency

Scalise G., Il mercato non basta. Attori, istituzioni e identità dell'Europa in tempo di crisi 

